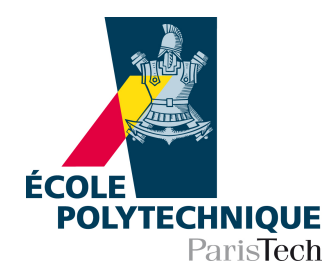

\title{
Applications of Reformulations in Mathematical Programming
}

\author{
Thèse présentée pour obtenir le grade de \\ DOCTEUR DE L'ÉCOLE POLYTECHNIQUE
}

par

Alberto Costa

Soutenue le 18 septembre 2012 devant le jury composé de:

$\begin{array}{lll}\text { Eligius M. T. Hendrix } & \text { Wageningen University, Wageningen } & \text { Rapporteur } \\ \text { Arnaud Pêcher } & \text { Université de Bordeaux I, Bordeaux } & \text { Rapporteur } \\ \text { Olivier Bournez } & \text { École Polytechnique, Palaiseau } & \text { Examinateur } \\ \text { A. Ridha Mahjoub } & \text { Université Paris Dauphine (Paris 9), Paris } & \text { Examinateur } \\ \text { Roberto Wolfler Calvo } & \text { Université Paris Nord (Paris 13), Paris } & \text { Examinateur } \\ \text { Pierre Hansen } & \text { GERAD, HEC, Montréal } & \text { Directeur de thèse } \\ \text { Leo Liberti } & \text { École Polytechnique, Palaiseau } & \text { Directeur de thèse } \\ \text { Ider Tseveendorj } & \text { UVSQ, Versailles } & \text { Directeur de thèse }\end{array}$



Premature optimization is the root of all evil.

Donald Ervin Knuth 


\section{Abstract}

Mathematical programming is a technique that can be used to solve real-world optimization problems, where one wants to maximize, or minimize, an objective function subject to some constraints on the decision variables. The key features of mathematical programming are the creation of a model for describing the problem (the so called formulation), and the implementation of efficient algorithms to solve it (also called solvers). In this thesis, we focus on the first point. More precisely, we study some problems arising from different domains, and starting from the most natural models for describing them, we propose alternative formulations, which share some properties with the original models but are somehow better (for instance in terms of computational time needed to obtain the solution by the solver). These new models are called reformulations. We follow the classification of reformulations proposed by Liberti in [Reformulations in Mathematical Programming: Definitions and Systematics, RAIRO-OR, 43(1):55-86, 2009]: exact reformulations (also called opt-reformulations), narrowings, relaxations. This thesis is concerned with three mathematical programming applications where the reformulation was crucial to obtain a good solution. The first problem tackled herein is graph clustering by means of modularity maximization. Since this problem is NP-hard, several heuristics are proposed. We focus on a divisive hierarchical algorithm which works by recursively splitting a cluster into two new clusters in an optimal way. This splitting step is performed by solving a convex binary quadratic program. This is reformulated exactly to a more compact form without changing the optimal solutions set (exact reformulation). We also evaluate the impact provided by the reduction of the number of symmetric global optima of the problem, which is also an important topic of the next part of this thesis. The computational times are considerably reduced with respect to the original formulation. The second problem tackled in the thesis is the Packing of Equal Circles in a Square (PECS), where one wants to place non-overlapping equal circles in a unit square in such a way as to maximize the common radius. One of the reasons why the problem is hard to solve is the presence of several symmetric optimal solutions, and consequently a very large Branch-and-Bound tree. Some of the symmetric optima are made infeasible by adjoining some Symmetry Breaking 
Constraints (SBCs) to the formulation, thereby obtaining a narrowing. Both computational time and size of the Branch-and-Bound tree outperform the ones provided by the original formulation. The third application considered in the thesis is that of computing the convex relaxation for multilinear problems, and to compare the "primal" formulation and another one obtained using a "dual" representation. Although these two relaxations are both already known in the literature, we make a striking observation, i.e., that the dual relaxation leads to a faster and more stable solution process as regards CPU time. 


\section{Résumé}

La programmation mathématique est une technique qui peut être utilisée pour résoudre des problèmes concrets où l'on veut maximiser, ou minimiser, une fonction objectif soumise à des contraintes sur les variables décisionnelles. Les caractéristiques les plus importantes de la programmation mathématique sont la création d'un modèle pour décrire le problème (aussi appelé formulation), et la mise en œuvre d'algorithmes efficaces pour le résoudre (aussi appelés solveurs). Dans cette thèse, on s'occupe du premier point. Plus précisemment, on étudie certains problèmes qui proviennent de domaines diffèrents, et en commençant par les modèles les plus naturels pour les décrire, on présente des formulations alternatives, qui partagent certaines propriétés avec le modèle original mais qui sont en quelque sorte meilleures (par exemple au niveau du temps d'exécution nécessaire pour obtenir la solution par le solveur). Ces nouveaux modèles sont appelés reformulations. On suit la classification des reformulations proposée par Liberti dans [Reformulations in Mathematical Programming: Definitions and Systematics, RAIRO-OR, 43(1):55-86, 2009] : exact reformulations (aussi appellées opt-reformulations), narrowings, relaxations. Cette thèse concerne trois applications de la programmation mathématique où les reformulations ont été fondamentales pour obtenir une bonne solution. Le premier problème étudié est le partitionnement de graphes sur la base de la maximisation de la modularité. Comme ce problème est NP-difficile, plusieurs heuristiques sont proposées. On s'occupe d'un algorithme séparatif hiérarchique qui fonctionne en divisant récursivement une classe en deux nouvelles classes de façon optimale. Cet étape de division est accomplie en résolvant un programme binaire quadratique et convexe. Il est reformulé de manière exacte pour obtenir une forme plus compacte sans modifier l'ensemble des solutions optimales (exact reformulation). On considère aussi l'impact donné par la réduction du nombre des solutions symétriques globalement optimales. Les temps d'exécution sont considérablement réduits par rapport à la formulation originelle. Le deuxième problème étudié dans cette thèse est le placement de cercles égaux dans un carré (Packing Equal Circles in a Square, ou PECS), où l'on veut placer des cercles égaux dans un carré de côté 1 sans avoir de superposition et en maximisant le rayon commun. L'une des raisons pour laquelle le problème est dif- 
ficile à résoudre vient de la présence de plusieurs solutions symétriques optimales, et par conséquent un arbre de séparation-et-évaluation (ou Branch-and-Bound) très large. Certaines solutions symétriques optimales sont rendues irréalisables en ajoutant des contraintes pour briser les symétries (Symmetry Breaking Constraints, ou $\mathrm{SBCs}$ ) à la formulation, en obtenant ainsi un narrowing. Le temps d'exécution et la dimension de l'arbre de Branch-and-Bound sont tous les deux meilleurs par rapport à la formulation originelle. La troisième application considérée dans cette thèse est le calcul de la relaxation convexe pour des problèmes multilinéaires, et la comparaison de la formulation "primale" avec celle obtenue par une représentation "duale". Bien que ces deux relaxations soient déjà connues, il est intéressant de voir que la relaxation duale conduit à des meilleures performances de calcul. 


\section{Acknowledgements}

I wish to thank several people. First of all, my supervisors: Pierre Hansen, Leo Liberti, and Ider Tseveendorj. They gave me the possibility to work on interesting topics, and to present my results in many interesting (and beautiful) places around the world. One of these is Paris, where I spent most of these three years. This experience was very important, both for my professional and personal life. All this was made possible by means of Digiteo's financial support under contract 2009-55D "ARM".

I would also like to thank my friends. Alena, because she allows me to see the world from another point of view. Fabio and Francesca, for their friendship and help, as well as for the poker tournaments. Alessandra, Álvaro, Anna, Arabella, Cesar, Claire, Claudia, David, Dimo, Dominik, Emanuele, Eugenio, Hassan, Irene, Jennifer, Jerome, Katya, Lorenzo, Lucio, Mahsa, Marc, Marco, Maria, Nives, Ryna, Sonia, Xue, and my friends in Italy.

Moreover, I thank my Master thesis' supervisor, prof. Massimo Melucci, for his suggestions and many interesting discussions.

Finally, I would like to thank my family for their support and love. 


\section{Acronyms}

- ASC: Almost-Strong Communities detection algorithm for clustering problem;

- BB: Branch-and-Bound;

- BMM: Bipartite Modularity Maximization;

- cMINLP: convex Mixed Integer Nonlinear Programming;

- cMIQP: convex Mixed Integer Quadratic Programming;

- cNLP: convex Nonlinear Programming;

- CPP: Circle Packing Problem;

- DAG: Directed Acyclic Graph;

- e.g.,: exempli gratia, in Latin. It means "for example";

- i.e.,: id est, in Latin. It means "that is";

- KKT: Karush-Kuhn-Tucker conditions;

- LP: Linear Programming;

- MILP: Mixed Integer Linear Programming (synonym of MIP);

- MINLP: Mixed Integer Nonlinear Programming;

- MIP: Mixed Integer Programming (synonym of MILP);

- MM: Modularity Maximization;

- MP: Mathematical Programming;

- NLP: Nonlinear Programming;

- PECS: Packing Equal Circles in a Square;

- PPS: Point Packing in a Square;

- QCQP: Quadratically Constrained Quadratic Problem;

- sBB: spatial Branch-and-Bound;

- SBC: Symmetry Breaking Constraint;

- SC: Strong Communities detection algorithm for clustering problem;

- SQP: Sequential Quadratic Programming;

- s.t.: subject to;

- WLOG: Without Loss Of Generality. 


\section{Contents}

1 Introduction $\quad 1$

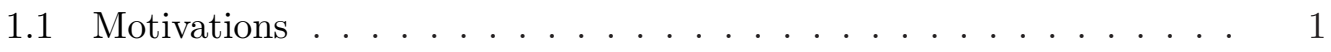

1.2 Mathematical programming . . . . . . . . . . . . . 3

1.2.1 Classification of mathematical programming problems . . . . 4

1.2.1.1 Convexity ................... 4

1.2.1.2 Classes of mathematical programming problems . . 6

1.2.2 Approaches to solve mathematical programming problems . . 7

1.2.2.1 Linear programming . . . . . . . . . . 8

1.2.2.2 Mixed integer linear programming . . . . . . . 8

1.2.2.3 Nonlinear and convex nonlinear programming . . . 11

1.2.2.4 Convex mixed integer nonlinear programming . . . 13

1.2.2.5 Mixed integer nonlinear programming . . . . . . . 14

1.3 Reformulations .................... . . 15

1.3.1 Classification of reformulations . . . . . . . . . 16

1.3.1.1 Exact reformulations ............ . 17

1.3.1.2 Narrowings ................. . . 17

1.3.1.3 Relaxations ................. 18

1.4 Contributions . . . . . . . . . . . . . 18

I An application of exact reformulations $\quad 21$

2 Clustering in general and bipartite graphs $\quad 25$

2.1 Definitions and notation . . . . . . . . . . 27

2.2 Clustering based on modularity maximization . . . . . . . . . . 28

2.2.1 Hierarchical divisive heuristic . . . . . . . . . . . 30

2.2.1.1 Reduction of number of variables and constraints . 33

2.2.1.2 Binary decompositions . . . . . . . . . 36

2.2.1.3 Symmetry breaking constraint . . . . . . . . . . 39

2.2.1.4 Numerical results ... . . . . . . . . . . . . 39 
2.2.2 Extension to bipartite graphs . . . . . . . . . . . . . . . 42

2.2.2.1 Fortet linearization . . . . . . . . . . . . 44

2.2.2.2 Square reformulation . . . . . . . . . . . 46

2.2.2.3 Binary decomposition . . . . . . . . . . . . . . . 48

2.2.2.4 Numerical results . . . . . . . . . . . . . . 49

2.3 Clustering based on strong and almost-strong conditions . . . . . . . 52

2.3.1 Strong communities detection . . . . . . . . . . . 55

2.3.2 Almost-strong communities detection . . . . . . . . . . 58

2.3.3 Comparison between SC and ASC . . . . . . . . . . . 60

2.4 Conclusions . . . . . . . . . . . . . . . . . . . . 64

\section{$\begin{array}{lll}\text { II An application of narrowings } & 67\end{array}$}

3 Circle packing in a square $\quad 71$

3.1 Mathematical programming formulations . . . . . . . . . . 75

3.2 Detection of symmetries for circle packing . . . . . . . . . 76

3.2 .1 Definitions and notation . . . . . . . . . . . . 80

3.2.2 Automatic symmetry detection . . . . . . . . . . . . 80

3.2.3 Symmetric structure of circle packing . . . . . . . . . . 82

3.3 Order symmetry breaking constraints . . . . . . . . . . . . . . 84

3.3.1 Weak constraints . . . . . . . . . . . . . . 84

3.3 .2 Strong constraints . . . . . . . . . . . . . . . . 84

3.3 .3 Mixed constraints . . . . . . . . . . . . . . 86

3.3 .4 Numerical results . . . . . . . . . . . . . . . . . . . . . . . 88

3.4 Other constraints . . . . . . . . . . . . . . . . . . . . 90

3.4.1 Fixing points symmetry breaking constraints . . . . . . . 90

3.4.2 Bounds symmetry breaking constraints . . . . . . . . . . 93

3.4 .3 Triangular inequality constraints . . . . . . . . . . . 94

3.4.4 Numerical results . . . . . . . . . . . . . . . . . . . . . . 95

3.5 A conjecture about the reduction of the search space . . . . . . . . 96

3.6 Conclusions . . . . . . . . . . . . . . . . . . . . . . . . . . . 99

$\begin{array}{ll}\text { III An application of relaxations } & 101\end{array}$

4 Primal and dual convex relaxations for multilinear terms $\quad 105$

4.1 Definitions and notation . . . . . . . . . . . . . 106

4.2 Primal relaxation . . . . . . . . . . . . . . . . . . 107

4.2 .1 Bilinear terms . . . . . . . . . . . . . . . . 108

4.2.1.1 McCormick's inqualities . . . . . . . . . . . . . 109

4.2.1.2 Fortet inequalities . . . . . . . . . . . . . 109 
4.2.2 Trilinear terms: Meyer-Floudas inequalities . . . . . . . . . 110

4.2 .3 Quadrilinear terms . . . . . . . . . . . . . . . . 111

4.3 Dual relaxation . . . . . . . . . . . . . . . . . . . 111

4.3 .1 Example... . . . . . . . . . . . . . . . . 112

4.4 Comparison and numerical results . . . . . . . . . . . . 113

4.5 Conclusions . . . . . . . . . . . . . . . . . . . . . . 114

$\begin{array}{lll}\text { IV Conclusions and bibliography } & 117\end{array}$

$\begin{array}{llr}5 & \text { Conclusions } & 119\end{array}$

$\begin{array}{lr}\text { Bibliography } & 123\end{array}$ 
Chapter 1

\section{Introduction}

\subsection{Motivations}

The aim of Mathematical Programming (MP) is to analyze and solve optimization problems. These involve the minimization (or maximization) of one (or possibly more) objective functions subject to some constraints expressed in terms of the decision variables. Several problems, arising from various domains (e.g., artificial intelligence [50,137], bioinformatics and computational biology [92, 113, 147, 150, 161, 162, 192-194, 249], chemistry and chemical engineering [14,111, 163, 167, 177], graph clustering [79,121], engineering [16,125, 226], location [41,120,146], medicine $[86,166,168,176,181,227]$, physics [149], transportation [12,21,229]), can be described in this way. Nevertheless, it is not always possible to easily solve such problems because of the size of the instances, nonlinearity and/or nonconvexity of the objective function and/or constraints, uncertainty in the input data, and other causes.

In the last decades the research carried out to solve more and more complex problems has followed two main directions: first, an improvement of the solvers and algorithms, taking also into account the increasing power of computers. Second, the way to model problems. These two aspects are in fact two sides of the same coin, since a good solution of an optimization problem is obtained by means of both an appropriate model (also called formulation) and an efficient algorithm to solve it. More precisely, the process which leads from a real-world problem to its solution by means of MP can be resumed in the following 4 steps, summarized in Figure 1.1:

1. formalize the (real-world) problem;

2. create an abstract mathematical model to describe the problem;

3. give the model as input to a solver in order to obtain the optimal solution (if the solution process is too much time and/or memory demanding due to the difficulty of the problem, and the optimal solution cannot be found, usually 
the solver can provide some other informations as the best solution found so far and sometimes a bound on the cost of the optimal solution);

4. interpret the solution within the real-world setting of the problem.

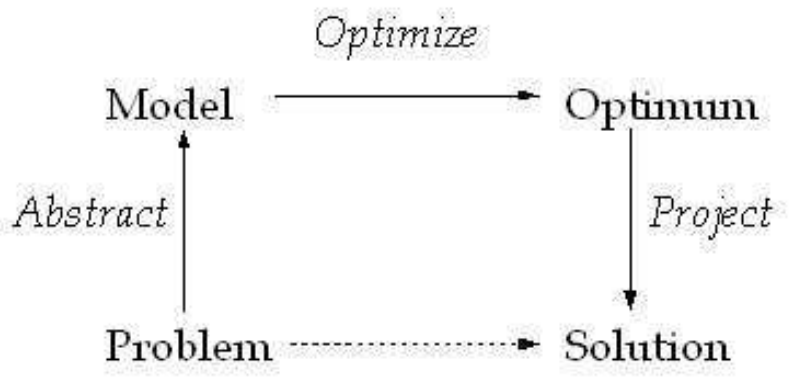

Figure 1.1: Solution process for a problem using MP (the picture is taken from http://www. eudoxus.com/).

Although the use of an efficient solver is very important, it is just as important to model the problem appropriately, as it directly affects solver's performance and the possibility to map the optimal solution into the real-world domain. Regarding the importance of solvers and computer power, and considering for instance linear optimization, during the Panel session of the $1^{\text {st }}$ International Conference on Operations Research and Enterprise Systems (ICORES) held in Portugal on February 2012, Dominique De Werra (professor at École Polytechnique Feredale de Lausanne and president of IFORS ${ }^{1}$ from 2010 to 2012) recalled that from 1988 to 2003 the improvement of computers power can be estimated as 800x, whereas the improvement of the efficiency of algorithms as $2.360 \mathrm{x}$, giving a global acceleration of almost two million fold. More details can be found in [30]. Note that in this thesis we mostly consider general-purpose solvers, and we focus on the design of efficient MP models. However, given a problem, one can design a specific algorithm to solve it, as done for example in Section 2.3.

Concerning the models, the most natural way to describe a problem often leads to a formulation which might not be the best for a given solver. Therefore, starting from a first formulation, one tries to modify it in order to obtain an alternative formulation, called reformulation, which is somehow better (for instance in term of computational time needed by the solver to obtain the optimal solution). Unlike the previous point about algorithms and computational power, it is not easy to estimate how much one can gain by reformulating a problem in the general case, since this depends on the problem itself and also on the features of the solver which can be exploited by the new formulation. Furthermore, after reformulating a problem, one

\footnotetext{
${ }^{1}$ IFORS is the International Federation of Operational Research Society; it has been founded in 1953 by UK, USA and France, and now counts more than 50 national societies. Its role is to promote the development of Operations Research worldwide.
} 
could employ alternative (and more efficient) solvers. For instance, if a nonlinear problem can be reformulated as a linear one, one may take advantage of powerful solvers such as CPLEX [135] or Gurobi [117], which are usually more robust than the nonlinear ones. For example, consider a problem which is nonlinear due to the presence of products between binary variables. It can be reformulated exactly (i.e., without changing the set of optimal solutions) as an integer linear problem by means of the Fortet's inequalities, which are introduced in Sections 2.2.1.1 and 4.2.1.2. However, given an optimization problem and a solution algorithm, there exists a formulation of the problem that is optimal with respect to the CPU time taken by the algorithm to solve it (again, in case of problems where the optimal solution cannot be found in a reasonable amount of time, other parameters can be considered, such as the best solution or the best bound found so far). The reformulated model should be as close as possible to this best formulation.

Another important application of reformulations arises when considering MP languages such as AMPL [97] or GAMS [42]. Each solution algorithm requires the problem to be cast in a particular form, called standard form; for instance, the simplex algorithm [70] requires linear equality constraints only with inequalities limited to the variable bounds. The reformulation of the problem into the standard form for the chosen solver is carried out automatically, thus the users are free to focus on modeling rather than worrying about algorithmic details. Other examples of automatic reformulations are presented in [7,158].

It turns out that the field of reformulations is very important and can have a high impact in both academia and industry. Thus, the motivations of this thesis are mainly two: first, to perform an analysis of different problems, trying to understand which is the best way to reformulate them, and moving toward the best formulation. Second, to show the impact of different reformulation techniques when applied to these problems.

The rest of this chapter is organized as follows: in Section 1.2 we present MP, while in Section 1.3 we introduce the theory and classification of reformulations, mainly based on the work presented in [157]. Finally, in Section 1.4 we summarize the main contributions of this thesis.

\subsection{Mathematical programming}

There exist several definitions of MP. One can simply state that MP is a branch of Operations Research which can be employed to analyze and solve real-world problems where one wants to maximize, or minimize, an objective function subject to some constraints on the decision variables. A more "applications-oriented" definition (related to the historical origin of MP as tool to solve problems arising in the army field), taken from [38], is the following: 
It concerns the optimum allocation of limited resources among competing activities, under a set of constraints imposed by the nature of the problem being studied. These constraints could reflect financial, technological, marketing, organizational, or many other considerations. In broad terms, mathematical programming can be defined as a mathematical representation aimed at programming or planning the best possible allocation of scarce resources.

Indeed, these definitions are not formal, but helpful to understand what is MP and what kind of problems it can deal with. Moving toward a more precise definition, we can express a generic MP formulation as:

$$
\begin{array}{ll}
\min & f(x) \\
\text { s.t. } & x \in X,
\end{array}
$$

where $X$ is the set of feasible solutions, and it is a cartesian product of continuous and discrete intervals (as it is defined by the constraints of the problem and the bounds on the variables), and $f: X \rightarrow \mathbb{R}^{F}$ represents the set of $|F|$ objective functions (if $|F|>1$ we have a multiobjective problem; in this thesis we always consider problems where $|F|=1$ ). The problem represented by the model (1.1) can be expressed as: find a point $x^{*} \in X$ (called optimal solution or global optimum) which minimizes the objective function $f(x)$, that is $\forall x \in X f\left(x^{*}\right) \leq f(x)$. In the rest of the thesis we consider as global optimum the solution $x^{*}$, and $f\left(x^{*}\right)$ its cost, so in this sense all the different solutions having as cost $f\left(x^{*}\right)$ are global optima. A point $\bar{x} \in X$ is called local optimum if $\exists \epsilon>0 \mid \forall x \in X,\|x-\bar{x}\| \leq \epsilon$ it holds that $f(\bar{x}) \leq f(x)$, i.e., there are not better solutions than $f(\bar{x})$ in the neighborhood of $\bar{x}$. If a problem does not admit any optimal solution, it is called infeasible problem, that is $X=\emptyset$. If there exist many optimal solutions, the standard general-purpose solvers usually only find one solution, though the modern solvers have options for finding more.

\subsubsection{Classification of mathematical programming problems}

In this section we propose a classification of MP problems. Before doing that, a very important concept must be introduced: convexity. Note that in the rest of this chapter we always refer to minimization problems. A maximization problem where one wants to maximize an objective function $f$ can be reformulated as a minimization problem by means of the relationship $\max f=-\min -f$.

\subsubsection{Convexity}

For a class of problems, namely convex problems in form of minimization, the set of global optima is the same as the set of local optima. Intuitively, they are easier to solve, since there is no need to continue the search for a global optimum after 
having found a local optimum, whilst in general this is not true. In order to define more formally convexity, some definitions (mostly taken from [87]) are introduced in the following:

Definition 1.2.1 (Convex combination [87]). The convex combination of $k$ points $x_{1}, \ldots, x_{k} \in \mathbb{R}^{n}$ is defined as $z=\sum_{i=1}^{k} \lambda_{i} x_{i}$, where $\forall i \in\{1, \ldots, k\} \lambda_{i} \geq 0$ and $\sum_{i=1}^{k} \lambda_{i}=1$. If $\lambda \in(0,1)^{k}$, then $z$ is called strict convex combination.

When $k=2$, the previous definition can be reformulated as follows: given two points $x, y \in \mathbb{R}^{n}$, its convex combination $z$ is defined as $z=\lambda x+(1-\lambda) y$ where $\lambda \in[0,1]$ (strict if $\lambda \in(0,1)$ ). For the sake of clarity, in the following definitions we consider the case when $k=2$.

Definition 1.2.2 (Convex set [87]). A set $X \subseteq \mathbb{R}^{n}$ is called convex if $\forall x, y \in X$, it holds that $X$ contains all the convex combinations $z$ of $x$ and $y$, that is $z=$ $\lambda x+(1-\lambda) y \in X, \forall \lambda \in[0,1]$.

It also holds that intersection of convex sets is a convex set. An example of convex and nonconvex sets is depicted in Figure 1.2.

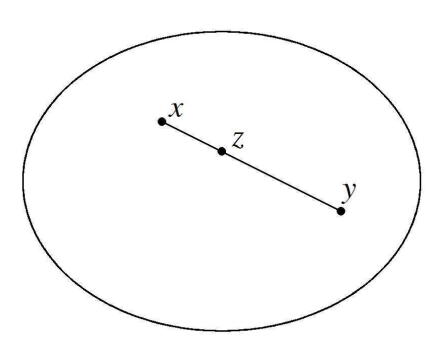

(a)

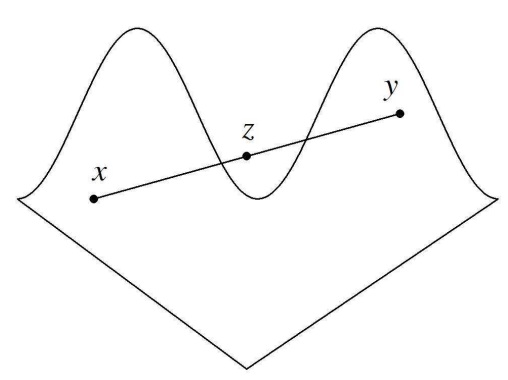

(b)

Figure 1.2: Examples of convex set (a) and nonconvex set (b).

Definition 1.2.3 (Convex function [87]). A function $f: X \rightarrow \mathbb{R}$ defined on a convex set $X \subseteq \mathbb{R}^{n}$ is called convex if $\forall x, y \in X, \forall \lambda \in[0,1]$, it holds that $f(z) \leq$ $\lambda f(x)+(1-\lambda) f(y)$, where $z=\lambda x+(1-\lambda) y$. If $\lambda \in(0,1)$ and $\forall x \neq y f(z)<$ $\lambda f(x)+(1-\lambda) f(y)$ then $f$ is called strict convex function.

A graphical representation of a convex function is given in Figure 1.3. It is interesting to underline some facts: (i) if a function $g(x)$ is convex, then the constraints having the form $g(x) \leq b, b \in \mathbb{R}$ are convex. In general, $g(x) \geq b$ could be a nonconvex constraint, even if $g(x)$ is a convex function. In the case of $g(x)$ convex, the constraint $g(x) \geq b$ is called reverse convex [240]; (ii) if $g(x)$ is linear, $g(x) O b$, where $O \in\{\leq,=, \geq\}$ is a convex constraint; (iii) if the set of feasible solutions $X$ is defined by convex constraints, then it is convex. We can now introduce the following theorem: 


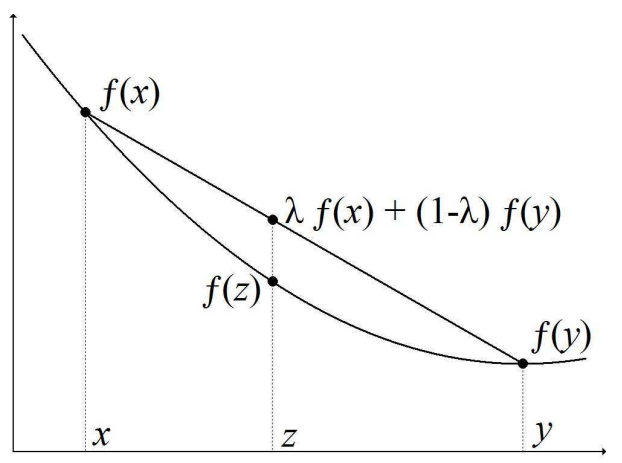

Figure 1.3: Convex function $f$

Theorem 1.2.4 (Property of optimal solutions for convex problems [87]). Consider a convex problem, that is a problem stated in the form (1.1) where $X \subseteq \mathbb{R}^{n}$ is a convex set, and the objective function to be minimized $f: X \rightarrow \mathbb{R}$ is a convex function. Each local optimum is also a global optimum.

Another important concept, that is concavity, is strictly related to convexity. More precisely, substituting $\leq$ and $<$ with $\geq$ and $>$ in Definition 1.2.3, we obtain the definitions of concave and strict concave function. These concepts are useful in the case of MP problem stated as maximization problems. The role of convexity and concavity in MP can be summarized by these facts [38]:

- A local minimum (maximum) of a convex (concave) function on a convex feasible region is also a global minimum (maximum).

- A local minimum (maximum) of a strict convex (concave) function on a convex feasible region is the unique global minimum (maximum).

\subsubsection{Classes of mathematical programming problems}

We can now propose a classification of the MP problems formulated in the very general form (1.1). Remember that the set $X$ is given by the bounds and kinds (as integer, continuous, or discrete) of the variables, and by the constraints of the problem, which are usually on the form $g(x) \leq 0$ or $g(x)=0$.

- Linear Programming (LP): the objective function and the constraints are linear, and the variables are continuous;

- Mixed Integer Linear Programming (MILP or MIP): the objective function and the constraints are linear, and at least one variable is integer; ${ }^{2}$

\footnotetext{
${ }^{2}$ Actually MILP is a special case of NLP, as the integrality of a variable $x_{j}$ can be expressed by the nonlinear constraint $\sin \left(\pi x_{j}\right)=0$. Nevertheless MILP is separated from NLP because there exist specific techniques to solve integer problems, as shown in Section 1.2.2.2. If all the variables are integer, sometimes ILP (Integer Linear Programming) is used in place of MILP to refer to the problem.
} 
- convex Nonlinear Programming (cNLP): the objective function and the constraints are convex with at least one of them being nonlinear, and the variables are continuous;

- Nonlinear Programming (NLP): at least one among the objective function and the constraints is nonlinear, and the variables are continuous;

- convex Mixed Integer Nonlinear Programming (cMINLP): the objective function and the constraints are convex with at least one of them being nonlinear, and at least one variable is integer;

- Mixed Integer Nonlinear Programming (MINLP): at least one among the objective function and the constraints is nonlinear, and at least one variable is integer.

We can further write the following relationships: LP $\subset$ MILP $\subset$ cMINLP $\subset$ MINLP and $\mathrm{LP} \subset \mathrm{cNLP} \subset \mathrm{NLP} \subset \mathrm{MINLP}$. The meaning is that if a solver can be employed for a given class of problems $C$, then it can also be employed for problems of all the classes $D \subset C$. For instance, a MINLP solver can be employed to solve a NLP problem, but a NLP solver working on a MINLP instance will ignore the integrality constraints on the variables. These relationships give also an intuitive idea about the complexity of the problems of the different categories. In general $D \subset C$ means that $D$ is easier to solve than $C$. Hence, LP problems are usually the easiest to solve, whereas MINLPs are the most difficult. Note that usually convex problems are easier to solve than nonconvex ones, since, in the former, local optima are also global optima, as explained earlier.

It is possible to go further into detail with the categorization of MP problems, but for this thesis the previous classification suffices. The clustering problems presented in Chapter 2 are MINLPs and cMINLPs, and we reformulate them as MILPs. In Chapter 3 the Packing Equal Circles in a Square (PECS) problem is an example of nonconvex NLP problem, and it is reformulated into another nonconvex NLP problem. Finally, the problems presented in Chapter 4 can be either MINLPs or NLPs, and they are reformulated respectively as MILPs and LPs.

At this point, the most natural questions are the following: which are the techniques used to solve these MP problems, and how the fact that a problem falls into one of the categories presented above affects the choice of the solution method? This is the subject of the next section.

\subsubsection{Approaches to solve mathematical programming problems}

In this section we present a brief summary of the techniques employed to solve MP problems belonging to the different classes presented in the previous section. If not specified, the variables are considered to belong to $\mathbb{R}$. 


\subsubsection{Linear programming}

In a LP problem the constraints and the objective function are linear. In its standard form, a LP problem can be expressed as:

$$
\begin{aligned}
\min & c^{T} x \\
\text { s.t. } & A x=b \\
& x \geq 0,
\end{aligned}
$$

where $c^{T}$ is the $n$ dimensional row vector of coefficients for the objective function, $A$ is the $m \times n$ matrix constraints, $b$ is the $m$ dimensional column vector representing the right-hand side of the constraints, and $x$ is the $n$ dimensional column vector of the nonnegative variables of the problem. The feasible region of such a problem is a convex set called convex polyhedron, having a finite number of vertices (i.e., points which cannot be expressed as strict convex combination of two any other points of the polyhedron). If the polyhedron is bounded it is called polytope. The importance of this concepts in LP is that the optimal solution of a LP problem corresponds to a vertex of the polytope representing the feasible region. This has been the key observation at the base of the simplex algorithm, that is an algorithm which starts from a vertex of the polyhedron and moves to another adjacent vertex as long as the objective function improves. The procedure stops when the vertex representing the optimal solution is reached. This is the main idea, but a lot of details are missed (e.g., how to perform this move from a vertex to a better one, how to know if the optimal vertex is found). For more informations, see [56,70]. Although this algorithm has an exponential complexity in the worst case, it is efficient in practice. However, in 1979 Khachiyan proved that LP can be solved in polynomial time, proposing the ellipsoid method, that is an interior point algorithm. In 1984 Karmarkar proposed a better polynomial time interior point method to solve LP problems [139]. The interior point methods are algorithms that find the optimal solution by moving on the interior of the polytope representing the feasible region, and not on the vertices as the simplex method. Regarding the efficiency, it is not clear which one between the simplex and the interior point algorithm performs better, since it depends on the problem itself. As consequence, LP solvers like CPLEX implement both methods. LPs are important because a lot of real-world problems can be described in this way. Moreover, LPs arise during the solution process of other categories of MP problems, as for example MILPs.

\subsubsection{Mixed integer linear programming}

A MILP problem consists of a linear objective function and some linear constraints, where a subset of the variables are integer. In general solving a MILP problem is NP-hard [101]. However, there is a special case where the optimal solution of a 
MILP problem can be obtained by relaxing the integrality constraints and solving the resulting LP problem (called continuous relaxation). Consider the MILP problem stated in the standard form as follows:

$$
\begin{array}{cl}
\min & c^{T} x \\
\text { s.t. } & A x=b \\
& x \in X \\
& \forall i \in I \quad x_{i} \in \mathbb{Z},
\end{array}
$$

where $I$ is the set of indices of integer variables. Let us introduce the concept of unimodularity:

Definition 1.2.5 (Unimodularity [87]). A $m \times n$ matrix $A$, where $m \leq n$, is called unimodular if for all $m \times m$ submatrices $B$ of $A$ it holds that $\operatorname{det}(B) \in\{-1,0,1\}$.

Suppose that the polyhedron defined by (1.3)-(1.4) is not empty and limited (i.e., it is a polytope). Then the Theorem 1.2.6 holds.

Theorem 1.2.6 (Integrality of the vertices of the polyhedron [87]). Let the $m \times n$ matrix $A$ be unimodular and the $m$ dimensional column vector $b$ be integer valued. The polyhedron associated to (1.3)-(1.4) has only integer vertices.

It is known that the optimal solution of a LP problem is found on a vertex of the polyhedron defined by the constraints of the problem. If we relax the integrality constraints (1.5) of the MILP problem, and solving the corresponding LP produces an integer solution, then this solution is optimal for the MILP problem. In other words, the unimodularity of the constraint matrix $A$ together with the integrality of the components of the vector $b$ is a sufficient condition for obtaining the optimal solution of the MILP problem by solving its continuous LP relaxation. In the case of problems where the constraints (1.3) are casted in form of inequalities, the concept of unimodularity has to be substituted with that of total unimodularity in order to preserve the property of having integer vertices of the polyhedron (the difference with respect to the unimodularity of Definition 1.2.5 is that, for total unimodularity, the property $\operatorname{det} B \in\{-1,0,1\}$ must hold for all $m \times m$ square submatrices $B$ of $A$ ).

In the general case, however, the solution obtained by solving the continuous relaxation of a MILP problem is not integer, hence other approaches must be employed. The main techniques are the following:

- Branch-and-Bound (BB) [148]: first the continuous relaxation of the MILP problem is solved. The optimal solution of the continuous relaxation $\check{x}$ has in general some components $\check{x_{i}}, i \in I$ which are not integer. Consider a fractional component $\check{x_{i}}$. Two new problems are generated from the original one, the first having adjoined the constraint $x_{i} \leq\left\lfloor\check{x}_{i}\right\rfloor$, the second having adjoined the 
constraint $x_{i} \geq\left\lceil\check{x}_{i}\right\rceil$. This step is called branching, and $x_{i}$ is the branching variable. The two subproblems generated correspond to express that $x_{i} \leq$ $\left\lfloor\check{x}_{i}\right\rfloor$ or $x_{i} \geq\left\lceil\check{x}_{i}\right\rceil$, that cannot be formulated by means of a linear constraint. Then the continuous relaxations of the two subproblems are solved. If each problem is represented by a node, each branching produces two children, and the resulting structure is a binary tree (usually called BB tree). For each node, after solving the corresponding continuous relaxation and obtaining the solution $\check{x}$, the process of branching and generation of the two child nodes is iterated unless one of the following fathoming criteria holds: (i) $\check{x}$ is integer; (ii) $\check{x}=+\infty$, i.e., the continuous relaxation of the problem is infeasible; (iii) $c^{T} \check{x} \geq c^{T} x^{*}$, where $x^{*}$ is the best optimal integer solution found so far (it is set to $+\infty$ at the beginning, and then updated each time a better integer solution is found). Note that $c^{T} \check{x}$ is a lower bound on the cost of the optimal integer solution which can be obtained by all the subproblems generated by the current node, i.e., these subproblems cannot provide solutions better than $c^{T} \check{x}$. This is the reason why it is not needed to continue the branching of a node if its continuous relaxation provides a solution that is worse than the best know integer solution. Two last details concern the choice of the branching variable, since in general there can be several variables in $I$ which are not integer, and the rule to explore nodes in the BB tree. A possible method to select the branching variable is to take the one having the fractional part closest to 0.5 , in order to reduce significantly the feasible region of both subproblems. Some well-known rules to select the node for performing the branching are a depth-first approach (where the node to process is the deepest node not yet explored), and a best-bound first approach (where the node to process is the one presenting the lower value of $c^{T} \check{x}$ ). When all the nodes are explored the BB returns the optimal solution $x^{*}$, if the problem is feasible.

- Cutting Plane [107]: the first step of this method is to solve the continuous relaxation of the problem. Then, given a solution $\check{x}$, one finds an inequality which is satisfied by each integer feasible solution of the problem but not by the current solution $\breve{x}$ (separation problem). This inequality, called cut, is adjoined to the MILP formulation and the continuous relaxation is solved again. This is repeated until the optimal solution is integer. Different types of cuts are provided in the literature. Some examples are represented by the Chvátal inequalities and the Gomory cuts.

- Branch-and-Cut $[203,204]$ : the problem of the cutting plane approach is that there can be several cuts that do not improve so much the current solution (tailing off). Thus, one can merge the $\mathrm{BB}$ and the cutting plane techniques. More precisely, at each node of the $\mathrm{BB}$ tree some cuts are adjoined to the model, in order to obtain a better lower bound (or ideally an integer solution), 
and consequently to employ in a more profitable way the fathoming criteria. When the cuts become no more effective, the branching is performed. This technique improves in general the results provided by BB or cutting plane used separately.

Heuristics algorithm are also very important, since they provide good feasible solutions which can be used to speed-up exact methods. Some examples are presented in $[28,68,88,89,106]$

\subsubsection{Nonlinear and convex nonlinear programming}

Nonlinear problems can be defined as follows:

$$
\begin{array}{ll}
\min & f(x) \\
\text { s.t. } & \forall i \in M \quad g_{i}(x) \leq 0 \\
& x \in X,
\end{array}
$$

where $M=\{1, \ldots, m\}$ and at least one among $g_{i}(x)$ and $f(x)$ is a nonlinear function. If there are no constraints on the variable, the problem is called unconstrained. Finding the optimal solution of a NLP problem is not as easy as for LP and MILP, due to the nonlinearities and in general nonconvexities (in this case Theorem 1.2.4 could not hold, with the possible consequence of having several local optima which makes the search for the global optimum by the solver difficult).

There exist some necessary conditions for the optimality called Karush-KuhnTucker (KKT) [140,144], which must be satisfied by a solution $x^{*}$ of a NLP problem to be a local optimum, and which are used by some NLP solvers. They can stated as follows:

Definition 1.2.7 (KKT conditions). Given a NLP problem in the form (1.6)(1.8), a feasible point $x^{*} \geq 0$ which respects some regularity conditions is a local optimum only if there exist some multipliers $\mu_{i}, \forall i \in M$ such that these conditions hold:

$$
\begin{array}{lll}
\forall i \in M & g_{i}\left(x^{*}\right) \leq 0 & \text { (primal feasibility) } \\
\mu_{i} \geq 0 & \text { (dual feasibility) } \\
\nabla f\left(x^{*}\right)+\sum_{i=1}^{m} \mu_{i} \nabla g_{i}\left(x^{*}\right)=0 & \text { (stationarity) } \\
\forall i \in M \quad \mu_{i} g_{i}\left(x^{*}\right)=0 & \text { (complementary slackness) }
\end{array}
$$

where the objective function $f$ and the constraints $g_{i}$ are differentiable in $x^{*}$ and the operator $\nabla$ applied to a function express its gradient. Some of the most common regularity conditions are called Linearly Independent Constraint Qualifications 
(LICQ) and require the gradient of the constraints that are active at $x^{*}$ to be linearly independent when evaluated at $x^{*}$.

In the case of convex objective function and constraints (that is a cNLP) a KKT point (i.e., a point which satisfies the KKT conditions) is a global optimum. Actually, this holds for a wider class of functions than convex ones, i.e., invex functions. For more details about invex functions, see $[27,65,122,123,180]$.

The main methods to solve NLPs are presented below. In the case of cNLPs the solution found is the global optimum. For nonconvex NLPs, some of these methods can be employed but there is not proof of global optimality for the solution found. To find an $\epsilon$ approximation of the global optimum for nonconvex NLPs, a possible approach is presented in Section 1.2.2.5.

- Line Search [23]: this is an iterative method to solve unconstrained NLPs. If the solution at the interation $t$ is $x^{t}$, the main steps for obtaining the new solution $x^{t+1}$ are: (i) find a descent direction, that is a vector representing the direction along which the objective function value decreases; (ii) decide a step size; (iii) let $x^{t+1}$ be equal to $x^{t}$ after the move of a step along the discent direction; (iv) repeat points (i)-(iii) until $\nabla f\left(x^{t+1}\right)$ is smaller than a given tolerance. There are several methods to decide the descent direction and the step size, e.g., gradient descent, Newton, Quasi-Newton, conjugate gradient.

- Trust Region [23]: this is another iterative method where a nonlinear function is not approximated in its whole domain, but only in a subset of the domain (called trust region) where the approximation is supposed to be good. This is done because the quality of the approximation of a nonlinear function near a given point could be not so good far from this point. The new solution $x^{t+1}$ is then searched within the trust region associated to the current solution $x^{t}$. There are different methods to decide the dimension of the trust region (defined by a step size), and the direction for the search.

- Penalty Function $[23,53]$ : in this case the constraints are removed from the problem and placed in the objective function in order to penalize solutions that do not respect the constraints. Thus, the problem to solve is an unconstrained problem.

- Interior Point $[23,53]$ : this method tries to reach the optimal solution by moving on the interior of the feasible region, unlike methods as the simplex for LPs, which moves on the boundary of the feasible region. This is done by means of barrier functions, which prevent leaving the feasible region.

- Sequential Quadratic Programming (SQP) [23,53]: differently from the penalty function and interior point approaches, this method tries to solve the KKT conditions for the original NLP problem. This leads to a quadratic 
problem, where the objective function, if nonlinear, is replaced by a quadratic approximation, and the nonlinear constraints are linearized. To obtain the optimal solution within a certain tolerance, a sequence of quadratic problems is solved.

\subsubsection{Convex mixed integer nonlinear programming}

To solve cMINLP problems the main approaches are the following:

- Branch-and-Bound [116]: this is the extension of the BB algorithm for MILP to nonlinear problems. At each node of the BB tree, instead of solving the LP problem corresponding to the continuous relaxation of a MILP problem, a cNLP problem (which corresponds to the continuous relaxation of a cMINLP problem) is solved.

- Outer-Approximation [81]: this is an iterative method where at each iteration a MILP relaxation of the cMINLP problem is solved (the nonlinear constraints are replaced by linear approximations). Then, the solution obtained is used to fix the integer variables of the cMINLP problem, and the corresponding cNLP relaxation is solved. The solution of the cNLP problem is used to generate some cuts to add to the MILP formulation, and the process is repeated. Solving the MILP problem provides a lower bound and solving the cNLP problem provides an upper bound on the solution of the cMINLP problem. When these two bounds are equal within a certain tolerance, then the optimal solution is found.

- Generalized Benders Decomposition [102]: this method is based on the Benders decomposition technique previously proposed by Benders for MILP. It can be seen as a variant of the outer-approximation method, where the MILP relaxation is not obtained by linearizing all the nonlinear constraints, but all these linearized constraints are combined to obtain a single constraint which is adjoined to the model (surrogate relaxation). As a consequence, the solution of this MILP problem provides in general a worse (i.e., lower) lower bound with respect to the outer-approximation method, leading to a larger number of iterations needed to obtain the solution, but on the other hand each MILP problem can be solved faster.

- Extended Cutting Plane [248]: this method works by solving iteratively a MILP relaxation of the original cMINLP problem, where the linearization of the most violated nonlinear constraint by the optimal solution is adjoined to the MILP formulation which is solved at the next iteration.

- LP/NLP based Branch-and-Bound [208]: this technique extends the outerapproximation approach in a branch-and-cut framework. More precisely, as in 
the outer-approximation method, a MILP relaxation is solved, but only once. In fact, this problem is solved by means of the BB as described for MILPs, with a main difference. Whenever an integer solution is found at the current node of the $\mathrm{BB}$ tree, it is used to fix the integer variables of the cMINLP problem yielding a cNLP problem. The solution of this cNLP problem is then used to derive some cuts that are adjoined to the MILP formulation at the current node, and the BB solution process continues.

As for MILPs, heuristics are very important for cMINLPs, since they can be used to find good feasible solutions and thus accelerate the algorithms presented above. Some examples are presented in $[1,29,34,35]$.

\subsubsection{Mixed integer nonlinear programming}

In the general case a MINLP problem is nonconvex. In this case, the use of the techniques employed for cMINLPs would provide a local optimum without proof of global optimality (unless the MINLP problem is reformulated as a cMINLP problem, but this is not always possible [153]). For obtaining global optimal solutions for nonconvex MINLPs, one can employ an $\varepsilon$-approximation algorithm called spatial Branch-and-Bound (sBB). Several variants exist, among which $[5,26,83,91,154,217$, 232, 242]. Couenne [26], or BARON [220] are examples of solvers implementing sBB. Given a constant $\varepsilon>0$, the sBB recursively generates a binary search tree, some leaf node of which contains a feasible point $x^{*}$ for which $f\left(x^{*}\right)$ differs by at most $\varepsilon$ from the globally optimal value of the objective function (with a slight abuse of notation, we refer to $x^{*}$ as the $\varepsilon$ approximation of the optimal solution instead of the real global optimum).

A very important step for each $\mathrm{sBB}$ algorithm is the convex relaxation of the original nonconvex problem. The solution of the convex relaxation provides a lower bound for the value of the optimal solution in the original problem. Some examples of convex relaxations are presented in Chapter 4, and more details about how these convex relaxations are computed are provided in [156]. At each iteration of the algorithm, convex relaxations restricted to particular sub-regions of space are solved, and a lower and an upper bound to the optimal value of the objective function can be assigned to each sub-region. A global optimum relative to the sub-region is identified when lower and upper bounds are very close together. More precisely, a generic node $a$ of the sBB tree contains a formulation restricted to some region, or box $B^{a}$ as well as a lower bound value $f\left(\check{x}_{a}\right)$ relative to the parent node. All along the sBB run, the following data are maintained:

- the search tree, encoded in some efficiently accessible form;

- the best solution so far (also called the incumbent). 
The following steps are performed at each node $a$. At the beginning, $f\left(x^{*}\right)=+\infty$ and $x^{*}=(+\infty, \ldots,+\infty)$.

1. Range tightening: techniques such as Optimization-Based Bounds Tightening (OBBT) [154] (where the range of variables is reduced in order to avoid the exploration of regions which do not contain any feasible point) and FeasibilityBased Bounds Tightening (FBBT) [24] (where using the constraints of the problem and interval analysis the bounds of the variables are tightened) are employed in order to attempt to reduce the width of $B^{a}$ in view to obtaining a tighter lower bound.

2. Computation of a lower bound $f\left(\check{x}_{a}\right)$ : this is done by means of solving a convex relaxation of the problem restricted to a region $B^{a}$.

3. Pruning by bound: if $f\left(\check{x}_{a}\right) \geq f\left(x^{*}\right)$ then the box $B^{a}$ cannot contain optima better than the incumbent. Go to Step 8.

4. Computation of an upper bounding solution $\left(\hat{x}_{a}, f\left(\hat{x}_{a}\right)\right)$ : it is obtained using a local NLP solver on the problem at the node, with $\left(\check{x}_{a}, f\left(\check{x}_{a}\right)\right)$ as a starting point.

5. Incumbent evaluation: if $f\left(\hat{x}_{a}\right)<f\left(x^{*}\right)$ then let $\left(x^{*}, f\left(x^{*}\right)\right) \leftarrow\left(\hat{x}_{a}, f\left(\hat{x}_{a}\right)\right)$.

6. Pruning by optimality: if $f\left(\hat{x}_{a}\right)-f\left(\check{x}_{a}\right)<\varepsilon$, then $\hat{x}_{a}$ is an $\varepsilon$-approximate global optimum within the box $B^{a}$; further refinements will not yield better optima. Go to Step 8.

7. Branching: select a variable and a value for branching: this consists in creating two subnodes $a_{1}, a_{2}$ of $a$, one with the subproblem where the branching variable is constrained between its lower range end and the branching value, and the other between the branching value and its upper range end; several heuristics exist for selecting branching variable and value [26].

8. Choice of next node: again, several heuristic methods exist. The most popular seems to be the choice of the node with the highest associated upper bound, insofar as it intuitively offers the best promise of improving the incumbent.

In the end, $x^{*}$ is the optimal solution. A proof of finite convergence of the sBB to an $\varepsilon$-approximation of a global optimum is given in [241].

\subsection{Reformulations}

In the literature, different definitions of reformulation are presented. For instance, Sherali proposed the following definition: 
Definition 1.3.1 (Sherali's reformulation [228]). A reformulation in the sense of Sherali of an optimization problem $P$ (with objective function $f_{P}$ ) is a problem $Q$ (with objective function $f_{Q}$ ) such that there is a pair $(\sigma, \tau)$ where $\sigma$ is a bijection between the feasible region of $Q$ and that of $P$, and $\tau$ is a monotonic univariate function with $f_{Q}=\tau\left(f_{P}\right)$.

This definition is really strict, excluding from the class of reformulations all the cases where there does not exist a bijection $\sigma$ between the feasible region of the reformulated problem $Q$ and that of the original one $P$.

An alternative definition of reformulation is given by Audet et al. in [15]:

Definition 1.3.2 (Audet's reformulation [15]). Let $P_{A}$ and $P_{B}$ be two optimization problems. A reformulation in the sense of Audet $B(\cdot)$ of $P_{A}$ as $P_{B}$ is a mapping from $P_{A}$ to $P_{B}$ such that, given any instance $A$ of $P_{A}$ and an optimal solution of $B(A)$, an optimal solution of $A$ can be obtained within a polynomial amount of time.

In this case the definition excludes nonpolynomial time reformulations, which could be carried out in a reasonable amount of time, and it includes all the polynomial time reformulations even if very slow in practice. Moreover, there is no guarantee of preserving local or global optima.

A third definition of reformulation (also called auxiliary problem) is due to Liberti [157]:

Definition 1.3.3 (Liberti's reformulation [157]). Any problem $Q$ that is related to a given problem $P$ by a computable formula $f(Q, P)=0$ is called an auxiliary problem (or reformulation) with respect to $P$.

Starting from this definition, four different types of reformulations are presented in [157]. We introduce them more in detail in the next section, except for the approximation reformulation that is not used in this thesis because an approximation just leads to one of the other types of reformulation for some limiting value of a parameter.

\subsubsection{Classification of reformulations}

Following the Definition 1.3.3, reformulations can be classified as:

- exact or opt-reformulations: transformations preserving all optimality properties;

- narrowings: transformations preserving at least one global optimum;

- relaxations: transformations based on dropping constraints, variable bounds or types;

- approximations: transformations that are one of the above types "in the limit". 
Given a problem, one could first try to obtain an exact reformulation, in order to have an alternative formulation which could be possibly easier to solve but preserving all optimality properties. If the problem is still hard to solve, and it presents several global optima, one can then try to obtain a narrowing. For very difficult problems, or for specific algorithms, it may be necessary to employ a relaxation, eliminating some constraints (e.g., integrality of variables, bounds on variables or some inequalities). Hence, the optimal solution of the relaxation provides a guaranteed bound to the optimal objective function value (lower bound in case of minimization, upper bound in case of maximization). In the worst case, one must employ approximations, which do not provide any guarantee on optimality.

We now introduce more formally these first three categories. We indicate as $\mathcal{F}(P), \mathcal{L}(P)$ and $\mathcal{G}(P)$ respectively the feasible region, the set of local optima, and the set of global optima for the problem $P$.

\subsubsection{Exact reformulations}

Exact reformulations are auxiliary problems that preserve all optimality information.

Definition 1.3.4 (Exact reformulation). $Q$ is an exact reformulation (or optreformulation) of $P$ if each local optimum $l \in \mathcal{L}(P)$ corresponds to a local optimum $l^{\prime} \in \mathcal{L}(Q)$ and each global optimum $g \in \mathcal{G}(P)$ corresponds to a global optimum $g^{\prime} \in \mathcal{G}(Q)$.

In other words, this type of reformulation preserves both local and global optimality informations. Exact reformulations can be chained (i.e., applied in sequence) to obtain other exact reformulations.

\subsubsection{Narrowings}

Narrowings are auxiliary problems where some global optima are removed, but at least one is kept.

Definition 1.3.5 (Narrowing reformulation). $Q$ is a narrowing of $P$ if each global optimum $g^{\prime} \in \mathcal{G}(Q)$ corresponds to a global optimum $g \in \mathcal{G}(P)$.

It turns out that there can be global optima in $\mathcal{G}(P)$ without any corresponding global optimum in $\mathcal{G}(Q)$. Narrowings are useful in presence of problems exhibiting many symmetries. For instance, the PECS problem presented in Chapter 3 has a high degree of symmetry, and the search tree associated to the sBB algorithm becomes very large. Hence, the time to reach the leaves (which represent the optimal solutions) can be prohibitive. In this case a narrowing, which can be obtained by adjoining Symmetry Breaking Constraints (SBCs) to the original formulation, can dramatically reduce the completion time. 
Note that exact reformulations can be seen as a special case of narrowings. Moreover, a narrowing chained to another narrowing leads another (more complex) narrowing, and a narrowing chained to an exact reformulation provides a narrowing.

\subsubsection{Relaxations}

A relaxation of a problem $P$ is an auxiliary problem $Q$ of $P$ whose optimal objective function value is a bound (lower in the case of minimization, upper in the case of maximization) for the optimum objective function value of the original problem. Such bounds are mainly used in BB type algorithms, which are the most common exact or $\varepsilon$-approximate (for a given $\varepsilon>0$ ) solution algorithms for MILPs, nonconvex NLPs and MINLPs. Moreover, these bounds can be used to evaluate the performance of heuristic algorithms without an approximation guarantee [72], or to guide heuristics [207].

Definition 1.3.6 (Relaxation). $Q$ is a relaxation of $P$ if $\mathcal{F}(P) \subseteq \mathcal{F}(Q)$, and considering minimization problems $P$ and $Q$ where $f_{P}$ and $f_{Q}$ are respectively their objective functions, then $\forall x \in \mathcal{F}(P), f_{Q}(x) \leq f_{P}(x)$.

In other words, a problem $Q$ is a relaxation of $P$ if both the feasible region of $P$ is contained into the feasible region of $Q$ and the objective function of $Q$ provides better (or equal) value than the objective function of $P$ when evaluated in the points of the feasible region of $P$.

There are different kinds of relaxations. For instance the elimination relaxation takes place when we simply drop some constraints (as in the continuous relaxation for integer problems, where the integrality constraints on the variables are dropped). In the surrogate relaxation a set of constraints is replaced by a linear combination of them. In the Lagrangian relaxation a set of constraints is removed from the model but the objective function is modified in order to penalize solutions which does not respect these constraints. A more detailed presentation of these relaxations can be found in [206].

Exact reformulations and narrowings are special types of relaxations. Furthermore, relaxations can be chained to obtain other relaxations, and chains of relaxations with exact reformulations and narrowings are themselves relaxations.

\subsection{Contributions}

The main goal of this thesis is to investigate problems to show the impact of the reformulations presented in Section 1.3. Rather than focusing on the design of specific algorithms for a given problem, we try to improve the MP model used to describe that problem, obtaining alternative models (reformulations), and comparing them with respect to the original formulation. This comparison very often takes into account the computation time, even if in some cases other parameters are considered 
(e.g., the quality of the partitions obtained by the algorithms proposed in Section 2.3, the effect of the SBCs on the results obtained by the local solver in Chapter 3, the value of the upper bound, the best solution found and the size of the BB tree for the PECS instances whose solution time reached the time limit, as presented in Table 3.6).

In Chapter 2 we introduce the problem of clustering in general and bipartite graphs as example of application of exact reformulations. We show that alternative formulations lead to an improvement of the computational time needed to get the solution. This chapter also contains an important contribution to clustering (albeit not strictly related to reformulations). Some of the models presented in that chapter contain simple SBCs, thus leading to narrowing reformulations. A more exhaustive analysis of narrowings is performed in Chapter 3, where we study the PECS problem. We consider this problem as example of the application of narrowings, because it involves a high degree of symmetry. We characterize the symmetric structure of the problem, and then we propose SBCs to remove some of the previously detected symmetries. Indeed, the problem is very difficult and we were not able to improve the best-known solutions (in terms of cost of the objective function), since the best results for large instances are often obtained by heuristics, and not by means of a MP model solved by a general MINLP solver (we employ the solver CouENNE for our tests). However, the impact of SBCs is very clear when comparing the number of sBB nodes and computational time for the original formulation and the narrowings.

Finally, Chapter 4 refers to relaxations. More precisely, we introduce problems with multilinear terms, and we propose two relaxations: one (called primal) obtained by replacing each multilinear term with a new variable and several constraints, and another one obtained using a dual representation. Even if the theory underlying this two relaxations is well-known, it is interesting to compare them empirically. It appears from our computational tests that the dual approach is more stable and outperforms the primal one in terms of computational time when the size of problems increases. This can have a considerable impact, since pratically every sBB code uses primal relaxations. 
Part I

An application of exact reformulations 

This part of the thesis is devoted to the problem of clustering in unweighted general and bipartite graphs, and it presents two main contributions. First, we introduce the concept of modularity as measure of quality for clustering, and we present an existing hierarchical divisive heuristic for finding high modularity partitions for a given graph, described in [47]. We propose several reformulations for the MP model used by this heuristic, which decrease the computational time. The proposed reformulations are mostly exact reformulations, even if there is also a SBC, which leads to narrowing reformulations. However, applications of narrowing will be studied in Chapter 3 . After that, we adapt the hierarchical divisive heuristic and the reformulations to bipartite graphs. This first part is mainly based on the work presented in $[45,58]$. In the second part we study clustering from another point of view, not strictly related with reformulations. More precisely, one can obtain partitions into clusters by specifying conditions that each cluster must respect. Starting from a previously proposed condition, namely the strong criterion of Radicchi et al. [210], we modify it obtaining the almost-strong criterion, that produces more informative partitions. We first present two MP models to describe the problem of finding partitions in the strong and almost-strong sense. However, due to the size of these formulations, we propose a specific algorithm to find these partitions. This second part is based on the work presented in [44]. 


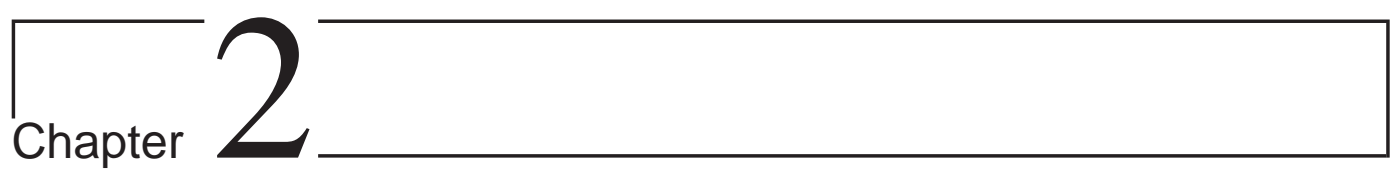

\section{Clustering in general and bipartite graphs}

Graphs have been intensively used in several domains to represent complex systems [198]. For instance, the metabolic networks studied in biology and bioinformatics [115, 205], social networks [105] and other applications in informatics, as recommender systems [6] or the World Wide Web [90]. One of the most important tasks is to identify the structure of such graphs, and in particular to find (generally disjoint) subsets of vertices, called communities or clusters, where each cluster contains vertices that are more likely to be pairwise connected with other vertices in the same cluster than with those belonging to other clusters. The detection of communities in graphs has many applications. The identification of relationships between users and products can be employed to develop targeted marketing programs or to design recommender systems that can suggest items to users, which is very useful for business purposes. Clustering is useful in biology, for example in the analysis of graphs representing interaction between proteins, to detect groups of proteins having similar functions within a cell. Another application arises from information retrieval, where clusters represent documents related to the same topic. This is a helpful support to search engines in the World Wide Web.

It often appears that complex and real-world graphs have a hierarchical structure, i.e., a cluster can be seen as a set of smaller clusters, and so on. Hierarchy in complex systems has been defined by H. A. Simon as follows [231]:

By a hierarchic system, or hierarchy, I mean a system that is composed of interrelated subsystems, each of the latter being, in turn, hierarchic in structure until we reach some lowest level of elementary subsystem.

Consider again the example taken from information retrieval: a cluster representing a set of documents related with a general topic (for example, cars), might contain smaller clusters, each one of them representing a more specific subject related with 
the topic of the parent cluster (for instance, different brands of cars like Renault, Citröen, Mazda, and so on). We might further suppose that each cluster representing a brand can be itself divided into other clusters, each one representing a specific model of car of that brand. Thus, clustering can also be employed to detect the hierarchical structure of a graph.

There are several methods to detect clusters in graphs, and they can be divided into three broad categories:

- Heuristic algorithms. In this case the clusters are found by a heuristic, as for example the hierarchical divisive heuristic proposed by Girvan and Newman [105], where the edge with largest betweenness (which is the number of pair of nodes for which the edge belongs to the shortest path joining them) is iteratively removed, and clusters correspond to connected components obtained each time a cluster is split in two. This heuristic therefore proceeds from an initial (trivial) partition in a single cluster containing all vertices to a final partition in which each cluster contains a single vertex.

- Maximization (or minimization) of an objective function. Among a large number of examples, one of the most known is the modularity, initially proposed as a stopping rule for the divisive heuristic mentioned above and later considered as an independent criterion; modularity is presented in detail in Section 2.2. Other well-known criteria are the $k$-way cut $[54,118]$, the normalized cut $[33,230]$, the ratio cut [118], the modularity density and its variants $[132,152]$, and strength maximization subject to strong or weak constraints on the communities $[78,185]$. More recently, several promising criteria have been put forward, e.g., information compression [216], maximum likelihood and the expectation maximization algorithm [17], and the constant Potts model [239].

- Constraints to be satisfied by each community. Several such constraints have been proposed; the early ones are reviewed in the book [245]. They include the cliques, in which every pair of vertices must be joined by an edge, the $k$-regular graph in which the indegree of each vertex must be at least $k$, and the LS (Luccio-Sami) set [174], i.e., a set of vertices $S$ such that each of its proper subsets has more ties to its complement within $S$ than to the outside of $S$. These three criteria tend to be too stringent and/or too difficult to compute, except on the smallest graphs. Two other well-known criteria, which express the intuitive idea of a community, have been proposed by Radicchi et al. [210]: a subset $S$ of vertices of a graph forms a community in the strong sense if the number of neighbors of each vertex within $S$ is larger than the number of neighbors outside $S$. A set of vertices $S$ forms a community in the weak sense if the sum, for all of its vertices, of the difference between 
the number of neighbors within $S$ and the number of neighbors outside $S$ is positive. Recently, weakened versions have been proposed, in which instead of comparing the numbers of neighbors within and outside the community, one compares the numbers of neighbors within a community, and outside that community but within another specific community [132]. In Section 2.3 we propose a weakened version of the concept of community in the strong sense, which leads to the definition of community in the almost-strong sense [44].

The main contributions of this chapter are the following: first, in Section 2.2.1 we introduce the hierarchical divisive heuristic presented in [47], and we propose some reformulations for the MP model used by this heuristic, which considerably reduce the computational time. In Section 2.2.2 we extend the divisive heuristic for the case of bipartite graphs, and we employ techniques similar to those presented in Section 2.2.1 to obtain good MP models for this heuristic. Finally, Section 2.3 deals with a contribution not related with modularity maximization and reformulations. Starting from the definition of community in the strong sense presented in [210], we propose a weakened version, yielding the so called community in the almost-strong sense, which appears to provide partitions into communities much more informative than the ones obtained by the original community in the strong sense criterion. In order to compare these criteria, two specific algorithms for finding partitions in the strong and almost-strong sense are proposed.

\subsection{Definitions and notation}

We denote a general graph, or network, by $G=(V, E)$, where $V$ is the set of $n$ vertices, and $E$ is the set of $m$ edges joining pairs of vertices. A vertex $v_{j}$ is represented by a point and an edge $e_{i, j}=\left\{v_{i}, v_{j}\right\}$ by a line joining its two end vertices $v_{i}$ and $v_{j}$. The shape of this line does not matter, only the presence or absence of an edge is important. A loop $e_{i, i}=\left\{v_{i}, v_{i}\right\}$ is an edge for which both end vertices coincide. In a simple graph, there is at most one edge between any pair of vertices, and no loops. The degree $k_{i}$ of a vertex $v_{i} \in V$ is the number of edges incident with $v_{i}$, and it can be split into two parts: the indegree $k_{i}^{i n}$ or number of neighbors of $v_{i}$ within its community and the outdegree $k_{i}^{\text {out }}$ or number of neighbors of $v_{i}$ outside its community. The adjacency matrix $A=\left(a_{i, j}\right)$ of $G$ is a square $n$ by $n$ matrix such that $a_{i, j}=1$ if vertices $v_{i}$ and $v_{j}$ are joined by an edge, and equal to 0 otherwise. A subgraph $G_{S}=\left(S, E_{S}\right)$ of a graph $G=(V, E)$ induced by a set of vertices $S \subseteq V$ is a graph with vertex set $S$ and edge set $E_{S}$ equal to all edges with both vertices in $S$. A set $S$ of vertices is a clique if all pairs of vertices of $S$ are joined by an edge, i.e., $\forall v_{i} \in S \quad k_{i}=|S|-1$. A set $S$ induces a $k$-regular graph if every vertex of $S$ has at least $k$ neighbors within $S$, where $k$ is a parameter.

A directed graph consists of a set of vertices and a set of oriented edges, called 
arcs. Unlike the undirected case, the $\operatorname{arcs}\left(v_{i}, v_{j}\right)$ and $\left(v_{j}, v_{i}\right)$ are not the same. In a weighted graph each edge is associated to a number, also called weight (in the unweighted graphs these weights can be considered 1 for each edge). A bipartite graph $G=\left(V_{R}, V_{B}, E\right)$, consists of two subsets of vertices $V_{R}=\left\{v_{1}, \ldots, v_{p}\right\}$ (called red vertices) and $V_{B}=\left\{v_{p+1}, \ldots, v_{n}\right\}$ (called blue vertices), and a set of edges $E$ connecting red and blue vertex pairs. Since there are no edges between two vertices $v_{i}$ and $v_{j}$ having the same color, the corresponding element $a_{i, j}$ of the adjacency matrix is 0 . Moreover, in an undirected graph $a_{i, j}=a_{j, i}$. Thus, the adjacency matrix $A_{b}$ of an undirected bipartite graph can be represented as:

$$
A_{b}=\left[\begin{array}{cc}
0_{p \times p} & \tilde{A}_{p \times q} \\
\left(\tilde{A}^{\mathrm{T}}\right)_{q \times p} & 0_{q \times q}
\end{array}\right] .
$$

Hence, the $p$ by $q$ matrix $\tilde{A}$ is sufficient to describe the graph completely.

A partition of a graph $G=(V, E)$ consist of a split of $V$ into pairwise disjoint nonempty clusters, or communities, $C_{1}, C_{2}, \ldots, C_{N c}$ that also cover $V$. This chapter deals with undirected unweighted general and bipartite graphs.

\subsection{Clustering based on modularity maximization}

Given a graph and a partition, a measure of the extent to which the classes of the partition can be considered to be communities is provided by the famous criterion called modularity $[105,199]$, which represents the fraction of edges within clusters minus the expected fraction of such edges in a random graph with the same degree distribution. Alternatively, given a graph, modularity can be maximized to find an optimal partition. Given an unweighted graph $G$, its modularity $Q$ is defined as:

$$
Q=\frac{1}{2 m} \sum_{i=1}^{n} \sum_{j=1}^{n}\left(A_{i, j}-\frac{k_{i} k_{j}}{2 m}\right) \delta\left(g_{i}, g_{j}\right),
$$

where $m$ is the number of edges of the graph, $g_{i}$ and $g_{j}$ are the clusters to which the vertices $v_{i}$ and $v_{j}$ belong, and $\delta\left(g_{i}, g_{j}\right)$ is the Kronecker symbol, equal to 1 if $g_{i}=g_{j}$, and 0 otherwise. Another equivalent definition of modularity is the following:

$$
Q=\sum_{c=1}^{N_{c}} Q_{c}=\sum_{c=1}^{N_{c}}\left(\frac{m_{c}}{m}-\frac{D_{c}^{2}}{4 m^{2}}\right),
$$

where $N_{c}$ is the number of clusters, $Q_{c}$ is the contribution to modularity of cluster $c, m_{c}$ is the number of edges within cluster $c, D_{c}$ is the sum of the degrees of the vertices which are inside the cluster $c, \frac{m_{c}}{m}$ is the fraction of edges in cluster $c$, and $\frac{D_{c}^{2}}{4 m^{2}}$ is the expected number of edges in cluster $c$ in a graph where vertices have the same degrees of distribution of $G$ but edges are placed randomly. The extension of this definition to weighted graphs is presented in [94]. The value of $Q$ is between $-\frac{1}{2}$ 
and 1; the lower bound is obtained for bipartite graphs if there are two clusters, one containing $V_{R}$ and the other one containing $V_{B}$. A value of 0 indicates a structure similar to a random graph, whereas a value close to 1 represents a graph with a strong community structure. It is important to underline that the value of $N_{c}$ is not known a priori. If $N_{c}=1$ then the modularity is equal to 0 , while a value of $n$ (one vertex per cluster) leads to a modularity smaller than 0 if there is at least one edge. In order to obtain good quality partitions, one should maximize the modularity; the corresponding problem is addressed as Modularity Maximization (MM). This is an NP-hard problem, as proved in [39].

Although modularity maximization is a very popular criterion, it presents some issues, the main ones being resolution limit and degeneracy. The former refers to the fact that in some cases small clusters may not be detected, and they remain hidden within another cluster, as reported in $[95,108]$. The latter is related to the possible presence of several high modularity partitions which makes it hard to find the global optimum [108]. Some methods to attenuate these issues are presented in $[13,145,213,221]$. However, modularity maximization remains a very interesting criterion for the detection of clusters; for the goal of this thesis, one of its most interesting properties is the fact that it can be described by means of mathematical programming. For a more detailed discussion of the strengths and weaknesses of modularity, see $[46,94,95]$.

For bipartite graphs, according to Barber [19] and to Leicht and Newman [151], the definition of modularity can be modified to obtain the bipartite modularity:

$$
Q_{b}=\frac{1}{m} \sum_{i=1}^{p} \sum_{j=p+1}^{n}\left(\tilde{A}_{i, j}-\frac{k_{i} k_{j}}{m}\right) \delta\left(g_{i}, g_{j}\right) \text {. }
$$

Again, it is possible to express it as the sum of the modularity for each cluster, obtaining:

$$
Q_{b}=\sum_{c=1}^{N_{c}} Q_{b_{c}}=\sum_{c=1}^{N_{c}}\left(\frac{m_{c}}{m}-\frac{R_{c} B_{c}}{m^{2}}\right),
$$

where $R_{c}$ represents the sum of the degrees of the red vertices in the cluster $c$, and $B_{c}$ is the sum of the degrees of the blue vertices in that cluster. Similarly to the previous case, the aim is to maximize bipartite modularity; we refer to this problem as Bipartite Modularity Maximization (BMM). In order to prove that this problem is NP-hard, in [252] the authors proposed a transformation from MM to BMM. Unfortunately, additional constraints were required, and the result is a problem which belongs to a different class of problems than modularity maximization in bipartite graphs, as shown in [58]. Thus, to the best of our knowledge, the complexity of BMM is an open problem.

In the literature, several methods have been proposed to find high modularity partitions for the MM problem: a few exact methods, and many heuristics. Among 
the exact methods, there is a clique partitioning formulation originally proposed by Grötschel and Wakabayashi [114], which is similar to the one presented by Brandes et al. [39], a convex Mixed Integer Quadratic Programming (cMIQP) formulation due to $\mathrm{Xu}$, Tsoka, and Papageorgiou [250], and the column generation extensions of these methods proposed by Aloise et al. [10]. Recently an improved version of the model proposed in [114], having a smaller set of inequalities, has been proposed in [76]. Concerning the heuristics, many algorithms have been proposed. Among the best known, there are heuristics based on simulated annealing [115, 182,184], mean field annealing [218], extremal optimization [80], spectral clustering [197,214,234], linear programming with randomized rounding [8], dynamical clustering [31], multilevel partitioning [77], contraction-dilation [186], multistep greedy search [222], quantum mechanics [200], label propagation [170], divisive and agglomerative approaches [47, 57,69, 195], and many others. For more details, see the survey of Fortunato [94]. Another interesting method, which improves the modularity obtained by heuristics by splitting and merging clusters, has been recently proposed in [48].

For bipartite modularity, among the best known heuristics there is the label propagation algorithm LPAb proposed by Barber and Clark [20] (which is an adaptation to bipartite case of the LPA algorithm proposed by Raghavan, Albert, and Kumara in [211]), the adaptive BRIM proposed by Barber [19], as well as the extension to bipartite graphs of the greedy agglomerative algorithm CNM of Clauset, Newman, and Moore [57], and the multistep greedy agglomerative algorithm MSG by Schuetz and Caflish [222,223]. Moreover, Liu and Murata proposed some extensions of label propagation algorithms: in [169], they presented a combination of LPA and BRIM (LP-BRIM), while in [171] they proposed a combination of LPAb and MSG, as well as LPAb+, that is a combination of a modified version LPAb, called LPAb' (where labels of blue and red vertices are not updated randomly as for LPAb, but by turn) and MSG. To the best of our knowledge, there are no exact algorithms for BMM.

In the first part of this chapter we focus on the divisive hierarchical heuristic presented by Cafieri, Hansen, and Liberti [47], which employs a MP model derived from that of $\mathrm{Xu}$, Tsoka, and Papageorgiou [250] when the number of clusters is 2 . We propose some reformulations for this model, in order to decrease the computational time. Moreover, we propose the extension of this heuristic for the bipartite case.

\subsubsection{Hierarchical divisive heuristic}

Clustering heuristics are either hierarchical, which aim at finding a set of nested partitions, or partitioning schemes, which aim at finding a single partition or possibly several partitions into given numbers of clusters. Hierarchical heuristics are in principle devised for finding a hierarchy of partitions implicit in the given graph when it corresponds to some situations where hierarchy is observed or postulated. This is often the case, for instance, in social organizations and evolutionary processes. 
Hierarchical heuristics can be further divided into agglomerative and divisive ones. Agglomerative approaches start from an initial partition where each vertex is associated to a cluster, and merge the closest ones in a bottom-up way. On the other hand, divisive heuristics proceed from an initial partition containing all the $n$ vertices of the graph and iteratively divide a cluster (usually into two new clusters) in such a way that the increase in the objective function value is the largest possible, or the decrease in the objective function value is the smallest possible [197]. Cluster bipartitions are iterated until a partition into $n$ clusters having each a single entity is obtained. In practice, with an objective function like modularity, bipartitions can be ended once they do not improve the objective function value anymore. A sketch of the divisive heuristic is given in Figure 2.1.

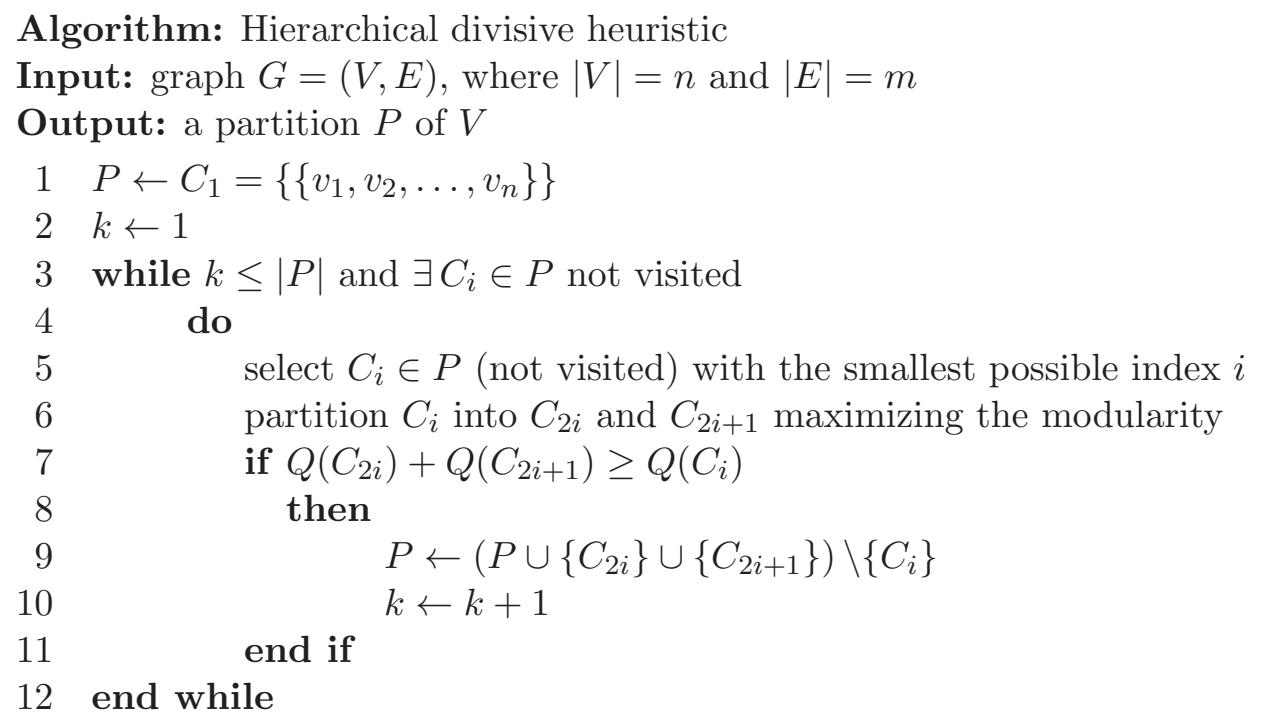

Figure 2.1: The hierarchical divisive heuristic.

Cafieri, Hansen, and Liberti [47] recently proposed a modularity maximizing divisive heuristic where the optimization subproblem for cluster bipartition is expressed as a cMIQP problem, using the model proposed in [250] with the number of clusters set to 2 . Binary variables are used to identify to which cluster each vertex and each edge belong. More precisely, variables $X_{i, j, s}$ for each edge $\left\{v_{i}, v_{j}\right\}$ and $s \in\{1,2\}$, and variables $Y_{i}$ for $i \in\{1,2, \ldots n\}$ are defined in such a way that $X_{i, j, s}$ is equal to 1 if the edge $\left\{v_{i}, v_{j}\right\}$ is inside the cluster $s$ (i.e., both vertices $v_{i}$ and $v_{j}$ are inside the cluster $s$ ), and $Y_{i}$ is equal to 1 if the vertex $v_{i}$ is inside the cluster 1 , and 0 otherwise. Moreover, the sets $V_{c}$ and $E_{c}$ are respectively the set of vertices of the cluster $c$ and the set of edges of the graph having both the end vertices in $V_{c}$.

Recall the definition of modularity (2.1). Since a bipartition has to be computed, only two sub-clusters have to be considered, and the sum of the degrees of the vertices belonging to one of the two sub-clusters can be expressed as a function of the sum 
of the degrees of the other cluster:

$$
D_{2}=D_{c}-D_{1}
$$

where $D_{1}$ and $D_{2}$ are the sums of the degrees of the vertices inside the two clusters and $D_{c}$ is a parameter given by the sum of degrees in the cluster $c$ to be bipartitioned (it is equal to $2 m$ at the outset). More precisely, $D_{c}$ is defined as:

$$
D_{c}=\sum_{v_{i} \in V_{c}} k_{i}
$$

where $k_{i}$ is the degree of the vertex $v_{i}$. Hence, in the bipartition subproblem the objective function (2.1) can be rewritten as:

$$
Q_{c}=\frac{m_{1}+m_{2}}{m}-\frac{D_{1}^{2}+D_{2}^{2}}{4 m^{2}}
$$

where $m_{1}$ and $m_{2}$ are respectively the number of edges inside the two clusters. Using equation (2.3), equation (2.4) can be rewritten as:

$$
Q_{c}=\frac{m_{1}+m_{2}}{m}-\frac{D_{1}^{2}+\left(D_{c}-D_{1}\right)^{2}}{4 m^{2}}=\frac{m_{1}+m_{2}}{m}-\frac{D_{1}^{2}}{2 m^{2}}-\frac{D_{c}^{2}}{4 m^{2}}+\frac{D_{1} D_{c}}{2 m^{2}} .
$$

As for the constraints, the following inequalities are used to impose that any edge $\left\{v_{i}, v_{j}\right\}$ with end vertices indexed by $i$ and $j$ can only belong to cluster $s$ if both of its end vertices also belong to that cluster:

$$
\begin{array}{ll}
\forall\left\{v_{i}, v_{j}\right\} \in E_{c} \quad X_{i, j, 1} \leq Y_{i} \\
\forall\left\{v_{i}, v_{j}\right\} \in E_{c} \quad X_{i, j, 1} \leq Y_{j} \\
\forall\left\{v_{i}, v_{j}\right\} \in E_{c} \quad X_{i, j, 2} \leq 1-Y_{i} \\
\forall\left\{v_{i}, v_{j}\right\} \in E_{c} \quad X_{i, j, 2} \leq 1-Y_{j} .
\end{array}
$$

Furthermore, the number of edges of each of the two clusters and the sum of the degrees of the vertices of the first cluster are expressed as follows:

$$
\begin{aligned}
& \forall s \in\{1,2\} \quad m_{s}=\sum_{\left\{v_{i}, v_{j}\right\} \in E_{c}} X_{i, j, s} \\
& D_{1}=\sum_{v_{i} \in V_{c}} k_{i} Y_{i} .
\end{aligned}
$$

Hence, the complete cMINLP formulation proposed in [47], and called from now $O B$ 
(Optimal Bipartition), is the following:

$$
\begin{array}{ll}
\max & \frac{1}{m}\left(m_{1}+m_{2}-\frac{1}{2 m}\left(D_{1}^{2}+\frac{D_{c}^{2}}{2}-D_{1} D_{c}\right)\right) \\
\text { s.t. } & \forall\left\{v_{i}, v_{j}\right\} \in E_{c} \quad X_{i, j, 1} \leq Y_{i} \\
& \forall\left\{v_{i}, v_{j}\right\} \in E_{c} \quad X_{i, j, 1} \leq Y_{j} \\
& \forall\left\{v_{i}, v_{j}\right\} \in E_{c} \quad X_{i, j, 2} \leq 1-Y_{i} \\
& \forall\left\{v_{i}, v_{j}\right\} \in E_{c} \quad X_{i, j, 2} \leq 1-Y_{j} \\
& \forall s \in\{1,2\} \quad m_{s}=\sum_{\left\{v_{i}, v_{j}\right\} \in E_{c}} X_{i, j, s} \\
& D_{1}=\sum_{v_{i} \in V_{c}} k_{i} Y_{i} \quad m_{s} \in \mathbb{R} \\
& \forall s \in\{1,2\} \quad m_{1} \in \mathbb{R} \quad X_{i, j, s} \in \mathbb{R}_{0}^{+} . \\
& \forall v_{i} \in V_{c} \quad Y_{i} \in\{0,1\} \\
& \forall\left\{v_{i}, v_{j}\right\} \in E_{c}, \forall s \in\{1,2\} \quad
\end{array}
$$

In order to solve $O B$, there are some possibilities:

1. employ general MINLP solvers, as Couenne [26] or BARON [220], or cMINLP solvers as BONMIN [36] since the problem is convex;

2. obtain an exact reformulation by linearizing the products between the binary variables $Y$ implied by $D_{1}^{2}$ using the Fortet inequalities [93];

3. use directly CPLEX [135], as $O B$ is a cMIQP problem (the only nonlinearity is the square in the objective function) that can be solved by CPLEX;

4. use the binary decomposition and then linearize the products between binary variables appearing in the resulting model.

Experiments showed that the first and second solutions were too much time consuming. Thus, only the last two possibilities are taken into account, and CPLEX is employed as solver.

Our goal is now to improve the formulation for the $O B$ model, as done for general graph partitioning problems in $[37,84]$. To do that, three techniques are analyzed and discussed separately in the rest of this section: (i) reduction of the number of variables and constraints; (ii) application of the binary decomposition technique; (iii) addition of a SBC.

\subsubsection{Reduction of number of variables and constraints}

Starting from the $O B$ model, half of the variables $X$ can be removed and the number of constraints can be reduced on the basis of the following considerations. 
Consider the $X$ variables. Looking at the objective function (2.5) of the $O B$ formulation, we notice that it contains the term $m_{1}+m_{2}$, which represents the number of edges in the first cluster plus the number of edges in the second one. Since we are interested in this sum, we do not actually need to know if an edge is in the cluster 1 or 2 , but only if it is within a cluster or not. Hence, we can drop the index $s$ of these variables, moving from the original definition:

$$
X_{i, j, s}= \begin{cases}1, & \text { if edge }\left\{v_{i}, v_{j}\right\} \text { belongs to cluster } s \\ 0, & \text { otherwise }\end{cases}
$$

to the following one:

$$
X_{i, j}= \begin{cases}1, & \text { if edge }\left\{v_{i}, v_{j}\right\} \text { is within cluster } 1 \text { or } 2, \\ 0, & \text { otherwise. }\end{cases}
$$

In other words, we can define $X_{i, j}$ as:

$$
X_{i, j}= \begin{cases}1, & \text { if } Y_{i}=Y_{j} \\ 0, & \text { otherwise }\end{cases}
$$

Since $X_{i, j}$ can be seen as the negation of the XOR operation between $Y_{i}$ and $Y_{j}$ variables, the following constraints can be employed [43]:

$$
\begin{array}{ll}
\forall\left\{v_{i}, v_{j}\right\} \in E_{c} \quad X_{i, j} \leq Y_{i}-Y_{j}+1 \\
\forall\left\{v_{i}, v_{j}\right\} \in E_{c} \quad X_{i, j} \leq Y_{j}-Y_{i}+1 \\
\forall\left\{v_{i}, v_{j}\right\} \in E_{c} \quad X_{i, j} \geq Y_{i}+Y_{j}-1 \\
\forall\left\{v_{i}, v_{j}\right\} \in E_{c} \quad X_{i, j} \geq 1-Y_{i}-Y_{j} .
\end{array}
$$

Note that, as in the original model, the $Y$ variables are binary and the $X$ variables are continuous. Moreover, only half of these constraints are useful: as explained in [2], the variables $X$ are maximized by the objective function, hence we only need 
(2.17)-(2.18). Therefore, we can reformulate the $O B$ model this way:

$$
\begin{array}{ll}
\max & \frac{1}{m}\left(\sum_{\left\{v_{i}, v_{j}\right\} \in E_{c}} X_{i, j}-\frac{1}{2 m}\left(D_{1}^{2}+\frac{D_{c}^{2}}{2}-D_{1} D_{c}\right)\right) \\
\text { s.t. } & \forall\left\{v_{i}, v_{j}\right\} \in E_{c} \quad X_{i, j} \leq Y_{i}-Y_{j}+1 \\
& \forall\left\{v_{i}, v_{j}\right\} \in E_{c} \quad X_{i, j} \leq Y_{j}-Y_{i}+1 \\
& D_{1}=\sum_{v_{i} \in V_{c}} k_{i} Y_{i} \\
& D_{1} \in \mathbb{R} \\
& \forall v_{i} \in V_{c} \quad Y_{i} \in\{0,1\} \\
& \forall\left\{v_{i}, v_{j}\right\} \in E_{c} \quad X_{i, j} \in \mathbb{R} .
\end{array}
$$

Due to the elimination of the index $s$ from the variables $X$, their number is now halved.

Consider again the definition (2.16) of the variables $X$. We can express it by employing the product of the variables $Y_{i}$ and $Y_{j}$ this way:

$$
X_{i, j}=2 Y_{i} Y_{j}-Y_{i}-Y_{j}+1
$$

Using this definition, we can replace the constraints (2.17)-(2.20) with a new set of inequalities, and replace the variables $X$ with another set of variables $S$ (having the same cardinality), which represent the product of the $Y$ variables in (2.28). These new variables $S$ are defined as:

$$
\forall\left\{v_{i}, v_{j}\right\} \in E_{c} \quad S_{i, j}=Y_{i} Y_{j}
$$

where the Fortet inequalities [93] can be used to describe this relationship (they can be also obtained after applying (2.28) to (2.17)-(2.20)):

$$
\begin{aligned}
& \forall\left\{v_{i}, v_{j}\right\} \in E_{c} \quad S_{i, j} \geq 0 \\
& \forall\left\{v_{i}, v_{j}\right\} \in E_{c} \quad S_{i, j} \geq Y_{j}+Y_{i}-1 \\
& \forall\left\{v_{i}, v_{j}\right\} \in E_{c} \quad S_{i, j} \leq Y_{i} \\
& \forall\left\{v_{i}, v_{j}\right\} \in E_{c} \quad S_{i, j} \leq Y_{j} .
\end{aligned}
$$

We can now replace the variables $X$ in the objective function (2.21) by means of the equation (2.28), and we can replace the constraints (2.22)-(2.23) with the new set (2.31)-(2.32) (again, we can drop the constraints (2.29) and (2.30) since we are 
maximizing the variables $S$ ). Thus, the new model, called $O B_{1}$, is the following:

$$
\begin{array}{ll}
\max & \frac{1}{m}\left(\sum_{\left\{v_{i}, v_{j}\right\} \in E_{c}}\left(2 S_{i, j}-Y_{i}-Y_{j}\right)+\left|E_{c}\right|-\frac{1}{2 m}\left(D_{1}^{2}+\frac{D_{c}^{2}}{2}-D_{1} D_{c}\right)\right) \\
\text { s.t. } & \forall\left\{v_{i}, v_{j}\right\} \in E_{c} \quad S_{i, j} \leq Y_{i} \\
& \forall\left\{v_{i}, v_{j}\right\} \in E_{c} \quad S_{i, j} \leq Y_{j} \\
& D_{1}=\sum_{v_{i} \in V_{c}} k_{i} Y_{i} \\
& \forall\left\{v_{i}, v_{j}\right\} \in E_{c} \quad S_{i, j} \in \mathbb{R} \\
& D_{1} \in \mathbb{R} \quad \\
& \forall v_{i} \in V_{c} \quad Y_{i} \in\{0,1\},
\end{array}
$$

where in (2.33) we use the fact that $\sum_{\left\{v_{i}, v_{j}\right\} \in E_{C}} 1=\left|E_{c}\right|$. Computational experiments show that the formulation using the $S$ variables outperforms the one with $X$ variables in terms of CPU time. Intuitively, constraints (2.34) and (2.35), which involve separately variables $Y_{i}$ and $Y_{j}$, give rise to a more sparse matrix constraints than the one associated with constraints (2.22)-(2.23) involving both $Y_{i}$ and $Y_{j}$.

\subsubsection{Binary decompositions}

The objective function of $O B$ involves the term $D_{1}^{2}$, which is the square of a sum of binary variables $Y$ multiplied by integer values, i.e., the degrees of the vertices. Hence, it is possible to apply the binary decomposition technique, also employed for general graph partitioning problems in [37], which consists in writing the term $D_{1}$ in this way:

$$
D_{1}=\sum_{l=0}^{t} 2^{l} a_{l}
$$

where $a_{l}$ are binary variables, and $t$ is a parameter which will be computed later. Using this definition of $D_{1}$, we can express $D_{1}^{2}$ as:

$$
D_{1}^{2}=\sum_{l=0}^{t} 2^{l} a_{l} \cdot \sum_{h=0}^{t} 2^{h} a_{h}=\sum_{l=0}^{t} \sum_{h=0}^{t} 2^{l+h} a_{l} a_{h}=\sum_{l=0}^{t} 2^{2 l} a_{l}+\sum_{l=0}^{t} \sum_{h<l} 2^{l+h+1} R_{l h},
$$

where $R_{l, h}$ are the variables used to replace the products between the variables $a_{l}$ and $a_{h}$. The Fortet inequalities can be used to express this relationship:

$$
\begin{array}{ll}
\forall l \in\{0, \ldots, t\}, \forall h \in\{0, \ldots, l-1\} & R_{l, h} \geq 0 \\
\forall l \in\{0, \ldots, t\}, \forall h \in\{0, \ldots, l-1\} & R_{l, h} \geq a_{l}+a_{h}-1 \\
\forall l \in\{0, \ldots, t\}, \forall h \in\{0, \ldots, l-1\} & R_{l, h} \leq a_{l} \\
\forall l \in\{0, \ldots, t\}, \forall h \in\{0, \ldots, l-1\} & R_{l, h} \leq a_{h} .
\end{array}
$$


Again, as for constraints (2.29)-(2.32), only half of the inequalities have been adjoined. This time, since the variables $R$ appear in the objective function with a negative sign, we should add (2.41) and (2.42) to the model. Finally, to estimate the parameter $t$ of (2.40), recall that the maximum value which can be taken by $D_{1}$ is the sum of the degrees of all the vertices in the current cluster $D_{c}$. Moreover, from (2.40) the maximum possible value for $D_{1}$ is $2^{t+1}-1$. Hence, $t$ can be computed as:

$$
2^{t+1}-1 \geq D_{c} \quad \Rightarrow \quad t=\left\lceil\log _{2}\left(D_{c}+1\right)-1\right\rceil .
$$

Now we can define the formulation $O B_{2 a}$ :

$$
\begin{aligned}
& \max \frac{1}{m}\left(m_{1}+m_{2}-\frac{1}{2 m}\left(\sum_{l=0}^{t} 2^{2 l} a_{l}+\sum_{l=0}^{t} \sum_{h<l} 2^{l+h+1} R_{l h}+\frac{D_{c}{ }^{2}}{2}-D_{1} D_{c}\right)\right) \\
& \text { s.t. } \forall\left\{v_{i}, v_{j}\right\} \in E_{c} \quad X_{i, j, 1} \leq Y_{i} \\
& \forall\left\{v_{i}, v_{j}\right\} \in E_{c} \quad X_{i, j, 1} \leq Y_{j} \\
& \forall\left\{v_{i}, v_{j}\right\} \in E_{c} \quad X_{i, j, 2} \leq 1-Y_{i} \\
& \forall\left\{v_{i}, v_{j}\right\} \in E_{c} \quad X_{i, j, 2} \leq 1-Y_{j} \\
& \forall l \in\{0, \ldots, t\}, \forall h \in\{0, \ldots, l-1\} \quad R_{l, h} \geq a_{l}+a_{h}-1 \\
& \forall s \in\{1,2\} \quad m_{s}=\quad \sum_{i, j, s} \\
& D_{1}=\sum_{v_{i} \in V_{c}} k_{i} Y_{i} \\
& D_{1}=\sum_{l=0}^{t} 2^{l} a_{l} \\
& \forall s \in\{1,2\} \quad m_{s} \in \mathbb{R} \\
&\left.D_{1} \in \mathbb{R} \quad \forall \quad \forall v_{i}\right\} \in E_{c} \\
& \forall v_{i} \in V_{c} \quad Y_{i} \in\{0,1\} \\
& \forall l \in\{0, \ldots, t\} \quad a_{l} \in\{0,1\} \\
& \forall\left\{v_{i}, v_{j}\right\} \in E_{c}, \forall s \in\{1,2\} \quad X_{i, j, s} \in \mathbb{R}_{0}^{+} \\
& \forall l \in\{0, \ldots, t\}, \forall h \in\{0, \ldots, l-1\} \\
& R_{l, h} \in \mathbb{R}_{0}^{+} .
\end{aligned}
$$

Note that $\mathbb{R}_{0}^{+}$is the set of real numbers greater than or equal to 0 , hence the constraint (2.60) implies also (2.41).

Compact binary decomposition It is possible to reduce the number of variables $R$ in the previous model. The variable $R_{l, h}$ is the linearization of the term $a_{l} a_{h}$, used in the objective function (2.46). We can write the part of this objective function 
which involves the variables $R_{l, h}$ in this way:

$$
\begin{aligned}
& \sum_{l=0}^{t} \sum_{h<l} 2^{l+h+1} R_{l h}=\sum_{l=0}^{t} \sum_{h<l} 2^{l+h+1} a_{l} a_{h}=\sum_{l=0}^{t} 2^{l+1} a_{l} \sum_{h<l} 2^{h} a_{h}= \\
& =\sum_{l=0}^{t} 2^{l+1} a_{l} b_{l}=\sum_{l=0}^{t} 2^{l+1} R_{l},
\end{aligned}
$$

where $R_{l}=a_{l} b_{l}$ and $b_{l}$ is a new variable defined as $\sum_{h<l} 2^{h} a_{h}$. Since the upper bound for $b_{l}$ is $U_{b_{l}}=\sum_{h<l} 2^{h}=2^{l}-1$, the constraints to add to the model are the following (they are derived from the McCormick's inequalities presented in Section 4.2.1.1):

$$
\begin{array}{ll}
\forall l \in\{0, \ldots, t\} \quad b_{l}=\sum_{h<l} 2^{h} a_{h} \\
\forall l \in\{0, \ldots, t\} \quad R_{l} \geq 0 \\
\forall l \in\{0, \ldots, t\} \quad R_{l} \geq U_{b_{l}} a_{l}+b_{l}-U_{b_{l}} .
\end{array}
$$

With respect to the previous formulation, we have now replaced the $\frac{t^{2}+t}{2}$ variables $R_{l, h}$ with $t+1$ variables $R_{l}$, and we have adjoined $t+1$ variables $b$, and $t+1$ constraints. Actually, we can notice that $b_{0}=0$ and $b_{1}=a_{0}$, but avoiding to define these variables does not change significantly the computation time. More in general, we can omit to define the variables $b$, since constraints $(2.62)$ can be removed and constraints (2.64) can be rewritten directly by replacing $b_{l}$ with $\sum_{h<l} 2^{h} a_{h}$. However, for the sake of the clarity, and to ease the explanation of the formulation presented in the following, we used the variables $b$. The formulation described in this section is addressed as $O B_{2 b}$.

Second compact binary decomposition Consider again the objective function (2.61) obtained after the transformation proposed in the previous section. In order to have a more compact representation of it, we can put together the terms containing the variables $a_{l}$ and $R_{l}$ in this way:

$$
\sum_{l=0}^{t} 2^{2 l} a_{l}+\sum_{l=0}^{t} 2^{l+1} R_{l}=\sum_{l=0}^{t} 2^{2 l} a_{l}+\frac{2^{2 l}}{2^{l-1}} R_{l}=\sum_{l=0}^{t} 2^{2 l}\left(a_{l}+\frac{a_{l} b_{l}}{2^{l-1}}\right) .
$$

Hence, we can write:

$$
\sum_{l=0}^{t} 2^{2 l}\left(a_{l}+\frac{a_{l} b_{l}}{2^{l-1}}\right)=\sum_{l=0}^{t} \frac{2^{2 l}}{2^{l-1}} a_{l}\left(b_{l}+2^{l-1}\right)=\sum_{l=0}^{t} 2^{l+1} a_{l} z_{l}=\sum_{l=0}^{t} 2^{l+1} T_{l},
$$

where the new variable $z_{l}$ is equal to $b_{l}+2^{l-1}$ and $T_{l}$ is the linearization of $a_{l} z_{l}$. Then, we should remove the variables $R$ and $b$ from the $O B_{2 b}$ formulation (and 
all the related constraints), and adjoin the new variables $z$ and $T$, as well as these constraints:

$$
\begin{array}{ll}
\forall l \in\{0, \ldots, t\} \quad z_{l}=\sum_{h<l} 2^{h} a_{h}+2^{l-1} \\
\forall l \in\{0, \ldots, t\} \quad T_{l} \geq 0 \\
\forall l \in\{0, \ldots, t\} \quad T_{l} \geq U_{z_{l}} a_{l}+z_{l}-U_{z_{l}}
\end{array}
$$

where $U_{z_{l}}$ is the upper bound of the variable $z_{l}$, and it is equal to $2^{l}-1+2^{l-1}$. The number of variables and constraints is the same as in the previous section (again, we could omit to define $z_{0}$ and $z_{1}$, since $z_{0}=2^{-1}$ and $z_{1}=a_{0}+1$, and we can omit to define the variables $z$ by expressing directly $z_{l}$ in the constraints $(2.67)$ thanks to equation (2.65)). The corresponding reformulation is called $O B_{2 c}$.

\subsubsection{Symmetry breaking constraint}

At each step of the algorithm a cluster is split into two new clusters, if this operation has the effect to increase the modularity. It is easy to see that, given a solution, the vertices in the first and second cluster can be swapped to obtain a symmetric solution. Since the problem of the optimal bipartitioning is solved exactly by the BB MIP algorithm of CPLEX, symmetric optima would lead to a large BB tree, and as consequence the time to reach the leaves of the tree (i.e., the optimal solutions) would increase. A simple way to avoid this is to fix one of the vertices to belong to one of the clusters.

Some tests show that best results are obtained by fixing the vertex with highest degree. Intuitively, this happens because that vertex is involved in more constraints. Hence, the model $O B_{3}$ is obtained by adding the following constraint to the model $O B$ :

$$
Y_{g}=0, g=\arg \max \left\{k_{i}, \forall v_{i} \in V_{c}\right\} .
$$

Note that, in case of multiple vertices having the same maximum degree, we set $g$ to be the smaller among the indices of these vertices.

\subsubsection{Numerical results}

In this section we present the comparison of the numerical results provided by the hierarchical divisive heuristic with the proposed reformulations. Results have been obtained on a $2.8 \mathrm{GHz}$ Intel Core i7 CPU of a computer with 8 GB RAM running Linux and CPLEX 12.2 [135], where we performed a fine tuning of the parameters (more precisely, after some tests we found as best configuration the one where we disabled the MIP cutting plane generation, and we used as branching variable selection strategy the branch based on pseudo reduced costs). Results are obtained on a set of instances of the literature, presented in Table 2.1. This set consists of these graphs: 
Zachary's karate club, describing friendship relationships in a karate club; Lusseau's dolphins, describing communications among a community of dolphins; Hugo's Les Misérables, representing relationships among characters in the book of Victor Hugo; A00 main and A01 main, showing classes and relationships from a software project related to graph drawing; p53, which shows protein interactions; Kreb's political books, representing books about US politics sold by Amazon; football, representing scheduling of football matches between American college teams; USAir97, describing connections between airports in the United States; netscience main, representing a coauhtorship graph between scientists; s838, describing electronic circuits; power, representing the topology of the power grid of the Western States of the United States.

In Tables 2.2-2.4 we show the comparison of the performances of the divisive hierarchical heuristic algorithm when the different proposed formulations for the bipartition model are used. $N_{c}$ denotes the number of clusters, $Q$ the modularity, and nodes the total number of BB nodes. Computing times are in seconds.

\begin{tabular}{rlrrl}
\hline ID & Graph & $\mathrm{n}$ & $\mathrm{m}$ & Reference \\
\hline 1 & karate & 34 & 78 & {$[251]$} \\
2 & dolphins & 62 & 159 & {$[175]$} \\
3 & Les misérables & 77 & 254 & {$[134,142]$} \\
4 & A00 main & 83 & 135 & {$[22]$} \\
5 & p53 protein & 104 & 226 & {$[71]$} \\
6 & political books & 105 & 441 & {$[22]$} \\
7 & football & 115 & 613 & {$[105]$} \\
8 & A01 main & 249 & 635 & {$[22]$} \\
9 & USAir97 & 332 & 2126 & {$[22]$} \\
10 & netscience main & 379 & 914 & {$[196]$} \\
11 & s838 & 512 & 819 & {$[190]$} \\
12 & power & 4941 & 6594 & {$[246]$} \\
\hline
\end{tabular}

Table 2.1: Informations about the graphs used for the tests.

It appears from Table 2.2 that the proposed reformulations of the original quadratic model clearly impact the resolution time and the number of nodes of the $\mathrm{BB}$ tree. $O B_{1}$ outperforms $O B_{3}$ in terms of computational time. As expected $O B_{3}$ reduces the number of $\mathrm{BB}$ nodes.

From Table 2.3 we note that when using the binary decomposition reformulations we obtain the best computational time with the $O B_{2 c}$ formulation (even if the number of nodes is larger), except for some of the largest instances (i.e., 7 (football), 9 (USAir97), and 12 (power)) where the best one is $O B_{2 a}$. Note that slight discrepancies may arise in the values of $N_{c}$ and $Q$; they are due to the fact that optimal bipartitions are not necessarily unique. For example, in the graph 6 (political books) there are differences between the results obtained by the binary reformulations and 


\begin{tabular}{rrrrrrrrr}
\hline & \multicolumn{1}{c}{$O{ }^{2}$} & \multicolumn{2}{c}{$O B$} & \multicolumn{2}{c}{$O B_{1}$} & \multicolumn{2}{c}{$O B_{3}$} \\
ID & $N_{c}$ & $Q$ & nodes & time & nodes & time & nodes & time \\
\hline 1 & 4 & 0.4188 & 45 & 0.14 & 41 & $\mathbf{0 . 0 6}$ & $\mathbf{1 8}$ & 0.07 \\
2 & 4 & 0.5265 & 207 & 0.59 & 157 & $\mathbf{0 . 1 9}$ & $\mathbf{9 8}$ & 0.49 \\
3 & 8 & 0.5468 & 205 & 1.09 & 185 & $\mathbf{0 . 4 0}$ & $\mathbf{1 0 2}$ & 0.58 \\
4 & 7 & 0.5281 & 76 & 0.35 & 56 & 0.11 & $\mathbf{2 7}$ & $\mathbf{0 . 0 8}$ \\
5 & 7 & 0.5284 & 275 & 1.10 & 201 & $\mathbf{0 . 5 3}$ & $\mathbf{1 3 5}$ & 0.59 \\
6 & 4 & 0.5263 & 313 & 3.04 & 294 & $\mathbf{1 . 0 0}$ & $\mathbf{1 4 5}$ & 1.36 \\
7 & 10 & 0.6009 & 8853 & 307.66 & 5410 & $\mathbf{5 6 . 6 9}$ & $\mathbf{3 0 1 4}$ & 118.24 \\
8 & 15 & 0.6288 & 1119 & 47.83 & 1010 & $\mathbf{1 6 . 8 5}$ & $\mathbf{9 9 7}$ & 45.85 \\
9 & 8 & 0.3596 & 16682 & 4585.04 & 17811 & $\mathbf{1 0 4 1 . 8 9}$ & $\mathbf{9 4 4 6}$ & 2510.81 \\
10 & 20 & 0.8470 & 291 & 3.64 & 267 & $\mathbf{1 . 4 4}$ & $\mathbf{1 0 8}$ & 1.82 \\
11 & 15 & 0.8166 & 392 & 5.26 & 304 & $\mathbf{1 . 2 6}$ & $\mathbf{1 9 7}$ & 2.15 \\
12 & 41 & 0.9396 & 1459 & 708.51 & 1449 & $\mathbf{2 1 7 . 6 1}$ & $\mathbf{8 1 5}$ & 417.26 \\
\hline
\end{tabular}

Table 2.2: Comparison between the original formulation $O B$ proposed in [47], the reformulation $O B_{1}$ with fewer variables and constraints, and $O B_{3}$ obtained by adjoining the SBC to the original formulation.

\begin{tabular}{rrrrrrrrr}
\hline & \multicolumn{1}{c}{$O B_{2 a}$} & \multicolumn{2}{c}{$O B_{2 b}$} & \multicolumn{2}{c}{$O B_{2 c}$} \\
ID & $N_{c}$ & $Q$ & nodes & time & nodes & time & nodes & time \\
\hline 1 & 4 & 0.4188 & $\mathbf{1 2 3}$ & 0.52 & 137 & 0.44 & 148 & $\mathbf{0 . 1 3}$ \\
2 & 4 & 0.5265 & 505 & 1.29 & $\mathbf{4 6 6}$ & 1.92 & 498 & $\mathbf{0 . 5 9}$ \\
3 & 8 & 0.5468 & 577 & 2.16 & 563 & 1.97 & $\mathbf{5 5 9}$ & $\mathbf{0 . 8 0}$ \\
4 & 7 & 0.5281 & $\mathbf{2 5 1}$ & 0.74 & 272 & 0.46 & 345 & $\mathbf{0 . 3 5}$ \\
5 & 7 & 0.5284 & $\mathbf{6 7 8}$ & 3.22 & 815 & 1.85 & 1052 & $\mathbf{1 . 3 8}$ \\
6 & 5 & 0.5270 & $\mathbf{1 2 8 4}$ & 9.17 & 1407 & 4.19 & 1670 & $\mathbf{3 . 9 9}$ \\
7 & 10 & 0.6009 & $\mathbf{2 5 4 0 6}$ & $\mathbf{2 5 2 . 9 6}$ & 40922 & 340.23 & 38910 & 331.50 \\
8 & 15 & 0.6288 & $\mathbf{4 3 9 5}$ & 61.49 & 5912 & 66.04 & 5783 & $\mathbf{5 8 . 7 3}$ \\
9 & 8 & 0.3596 & $\mathbf{6 3 6 8 7}$ & $\mathbf{3 0 7 4 . 0 9}$ & 89520 & 4295.85 & 91917 & 4610.60 \\
10 & 20 & 0.8470 & $\mathbf{9 3 1}$ & 14.53 & 1206 & 9.46 & 1359 & $\mathbf{7 . 1 7}$ \\
11 & 15 & 0.8167 & $\mathbf{1 3 4 8}$ & 22.46 & 2032 & 24.08 & 2317 & $\mathbf{1 1 . 3 1}$ \\
12 & 41 & 0.9395 & $\mathbf{1 1 2 8 9}$ & $\mathbf{2 0 2 9 . 6 3}$ & 16940 & 2605.25 & 19672 & 3071.16 \\
\hline
\end{tabular}

Table 2.3: Comparison between the different binary decomposition reformulations.

the other ones, and for the graph 12 (power) reformulations $O B_{2 b}$ and $O B_{2 c}$ provide 40 clusters instead of 41 , even if not reported in Table 2.3. The interest of reformulations based on binary decomposition, which lead to MILP models, will be evident in Section 2.2.2, when studying the BMM problem. Note that with different setting of the parameters and earlier versions of CPLEX, the best results (but still worse than those presented in Table 2.4) were obtained by the binary decomposition reformulation $O B_{2 c}$ together with the techniques used in $O B_{1}$ and $O B_{3}$.

In Table 2.4 we present the best results obtained by merging $O B_{1}$ and $O B_{3}$, that 


\begin{tabular}{rrrrrrrrr}
\hline & & & & \multicolumn{3}{c}{$O B$} & \multicolumn{2}{c}{$O B_{1}+O B_{3}$} \\
ID & $N_{c}^{*}$ & $Q^{*}$ & $N_{c}$ & $Q$ & nodes & time & nodes & time \\
\hline 1 & 4 & 0.4198 & 4 & 0.4188 & 45 & 0.14 & $\mathbf{1 7}$ & $\mathbf{0 . 0 4}$ \\
2 & 5 & 0.5285 & 4 & 0.5265 & 207 & 0.59 & $\mathbf{9 3}$ & $\mathbf{0 . 1 6}$ \\
3 & 6 & 0.5600 & 8 & 0.5468 & 205 & 1.09 & $\mathbf{1 0 5}$ & $\mathbf{0 . 3 5}$ \\
4 & 9 & 0.5309 & 7 & 0.5278 & 76 & 0.35 & $\mathbf{2 6}$ & $\mathbf{0 . 0 4}$ \\
5 & 7 & 0.5351 & 7 & 0.5284 & 275 & 1.10 & $\mathbf{1 1 9}$ & $\mathbf{0 . 2 6}$ \\
6 & 5 & 0.5272 & 4 & 0.5263 & 313 & 3.04 & $\mathbf{1 5 2}$ & $\mathbf{0 . 5 1}$ \\
7 & 10 & 0.6046 & 10 & 0.6009 & 8853 & 307.56 & $\mathbf{3 8 2 2}$ & $\mathbf{4 4 . 3 8}$ \\
8 & 14 & 0.6329 & 15 & 0.6288 & 1119 & 47.83 & $\mathbf{7 2 6}$ & $\mathbf{9 . 7 2}$ \\
9 & 6 & 0.3682 & 8 & 0.3596 & 16682 & 4585.04 & $\mathbf{8 6 6 5}$ & $\mathbf{4 4 6 . 0 6}$ \\
10 & 19 & 0.8486 & 20 & 0.8470 & 291 & 3.64 & $\mathbf{9 4}$ & $\mathbf{0 . 8 5}$ \\
11 & 12 & 0.8194 & 15 & 0.8166 & 392 & 5.26 & $\mathbf{1 8 6}$ & $\mathbf{1 . 1 8}$ \\
12 & - & - & 41 & 0.9396 & 1459 & 708.51 & $\mathbf{8 9 1}$ & $\mathbf{1 2 3 . 8 5}$ \\
\hline
\end{tabular}

Table 2.4: Optimal solutions $\left(N_{c}{ }^{*}\right.$ and $\left.Q^{*}\right)$ obtained by the column generation approach presented in [10], and comparison between the results obtained by the original formulation and the formulation $O B_{1}$ with fewer variables and constraints, together with the SBC of formulation $O B_{3}$.

is the compact reformulation of the original quadratic model with SBC adjoined. Both the computing time and the number of nodes are significantly reduced with respect to the original formulation. The computing time is reduced by a factor up to 10 for one of the largest instance, that is the number 9 (USAir97).

\subsubsection{Extension to bipartite graphs}

It is possible to extend the heuristic presented in previous section for the case of bipartite graphs, where we want to maximize the bipartite modularity (2.2). As in the previous case, the heuristic solves at each step a problem of optimal partitioning where the number of clusters is equal to 2 . We modify the best model obtained for the unipartite case, namely the $O B_{1}+O B_{3}$, adapting it for the bipartite case. First at all, we should define some parameters and variables. The variables $Y$ and $S$, as well as parameters $m$ and $k$, have the same meaning as for the MM problem. Parameter $D_{c}$ and variables $D_{1}$ and $D_{2}$ are no longer valid, thus we must modify them. Since the first $p$ vertices are red, and the other $n-p$ vertices are blue, we can define the two sets of blue and red vertices:

$$
\begin{aligned}
V_{R_{c}} & =\left\{v_{1}, \ldots, v_{p}\right\} \\
V_{B_{c}} & =\left\{v_{p+1}, \ldots, v_{n}\right\} .
\end{aligned}
$$

We should also define these two parameters: 


$$
\begin{aligned}
& R_{c}=\sum_{v_{i} \in V_{R_{c}}} k_{i} \\
& B_{c}=\sum_{v_{j} \in V_{B_{c}}} k_{i},
\end{aligned}
$$

which represent respectively the sum of the degrees of the red and blue vertices in cluster $c$, and they are known before the bipartition. Moreover, we shall define the following variables:

$$
\begin{aligned}
& R_{1}=\sum_{v_{i} \in V_{R_{c}}} k_{i} Y_{i} \\
& B_{1}=\sum_{v_{j} \in V_{B_{c}}} k_{j} Y_{j} .
\end{aligned}
$$

Furthermore, the following relationships hold:

$$
\begin{aligned}
& R_{c}=R_{1}+R_{2} \\
& B_{c}=B_{1}+B_{2} .
\end{aligned}
$$

The variables $R_{1}$ and $R_{2}$ represent, respectively, the sum of the degrees of the red vertices in the clusters 1 and 2 obtained by splitting the cluster $c$, while $B_{1}$ and $B_{2}$ are the same quantities for blue vertices.

All these new parameters and variables are related to the corresponding ones of MM by means of these relationships:

$$
\begin{aligned}
& V_{c}=V_{R_{c}} \cup V_{B_{c}} \\
& D_{c}=R_{c}+B_{c} \\
& D_{1}=R_{1}+B_{1} .
\end{aligned}
$$

Since the number of clusters is 2, using the new variables and parameters introduced above we can express the bipartite modularity (2.2) in this way:

$$
\begin{aligned}
Q_{b_{c}} & =\frac{m_{1}+m_{2}}{m}-\frac{R_{1} B_{1}+R_{2} B_{2}}{m^{2}}=\frac{m_{1}+m_{2}}{m}-\frac{R_{1} B_{1}+\left(R_{c}-R_{1}\right)\left(B_{c}-B_{1}\right)}{m^{2}}= \\
& =\frac{m_{1}+m_{2}}{m}-\frac{2 R_{1} B_{1}-B_{c} R_{1}-R_{c} B_{1}+R_{c} B_{c}}{m^{2}} .
\end{aligned}
$$


Hence, we can define the model $B O B$ (Bipartite Optimal Bipartition) as:

$$
\begin{array}{rlr}
\max & \frac{1}{m}\left(\sum_{\left\{v_{i}, v_{j}\right\} \in E_{c}}\left(2 S_{i, j}-Y_{i}-Y_{j}\right)+\left|E_{c}\right|-\frac{1}{m}\left(2 R_{1} B_{1}-B_{c} R_{1}-R_{c} B_{1}+R_{c} B_{c}\right)\right) \\
\text { s.t. } & \forall\left\{v_{i}, v_{j}\right\} \in E_{c} \quad S_{i, j} \leq Y_{i} \\
& \forall\left\{v_{i}, v_{j}\right\} \in E_{c} \quad S_{i, j} \leq Y_{j} \\
& R_{1}=\sum_{v_{i} \in V_{R_{c}}} k_{i} Y_{i} \\
& B_{1}=\sum_{v_{j} \in V_{B_{c}}} k_{j} Y_{j} \\
& Y_{g}=1, g=\arg \max \left\{k_{i}, \forall v_{i} \in V_{c}\right\} \\
& R_{1} \in \mathbb{R} \\
& B_{1} \in \mathbb{R} \\
& \forall\left\{v_{i}, v_{j}\right\} \in E_{c} \quad S_{i, j} \in \mathbb{R} \\
& \left.\forall v_{i} \in V_{c} \quad Y_{i} \in\{0,1\} .72 .76\right) \\
\end{array}
$$

Note that the SBC (2.81) fix the variable $Y_{g}$ (associated to the vertex with the largest degree) to 1 , instead of 0 as done for $\mathrm{MM}$ in equation (2.68). Experiments showed that this choice is more effective to break symmetries for bipartite graphs.

Looking at the objective function of the model, it is clear that it is not possible to employ CPLEX, since we have now the product $R_{1} B_{1}$, and not a square as in the unipartite case. Therefore, we have four possible ways to solve $B O B$ :

1. employ general MINLP solvers, as COUENNE or BARON;

2. linearize the products of binary variables $Y$ arising from $R_{1} B_{1}$ using the Fortet inequalities;

3. reformulate the problem in order to obtain a cMIQP model, and solve it with CPLEX;

4. use the binary decomposition and then linearize the products appearing in the resulting model.

As for MM it is too much time expensive to employ general MINLP solvers, so this solution will not be considered.

\subsubsection{Fortet linearization}

Considering the linearization by means of the Fortet inequalities [93], we have to replace the products between $Y$ variables in $R_{1} B_{1}$ using a new set of variables $W$, 
and to add some constraints. More precisely, the product $R_{1} B_{1}$ can be written as:

$$
R_{1} B_{1}=\sum_{v_{i} \in V_{R_{c}}} k_{i} Y_{i} \sum_{v_{j} \in V_{B_{c}}} k_{j} Y_{j}=\sum_{v_{i} \in V_{R_{c}}} \sum_{v_{j} \in V_{B_{c}}} k_{i} k_{j} Y_{i} Y_{j}=\sum_{v_{i} \in V_{R_{c}}} \sum_{v_{j} \in V_{B_{c}}} k_{i} k_{j} W_{i, j},
$$

where the variables $W_{i, j}$ are defined by these constraints (again, since these variables appear with negative sign inside an objective function to be maximized, only two constraints are needed):

$$
\begin{array}{ll}
\forall v_{i} \in V_{R_{c}}, \forall v_{j} \in V_{B_{c}} & W_{i, j} \geq 0 \\
\forall v_{i} \in V_{R_{c}}, \forall v_{j} \in V_{B_{c}} & W_{i, j} \geq Y_{i}+Y_{j}-1 .
\end{array}
$$

Hence, we obtain the model $B O B_{1 a}$ :

$$
\begin{aligned}
\max & \frac{1}{m}\left(\sum_{\left\{v_{i}, v_{j}\right\} \in E_{c}}\left(2 S_{i, j}-Y_{i}-Y_{j}\right)+\left|E_{c}\right|-\frac{1}{m}\left(2 \sum_{v_{i} \in V_{R_{c}}} \sum_{v_{j} \in V_{B_{c}}} k_{i} k_{j} W_{i, j}+\right.\right. \\
& \left.\left.-B_{c} R_{1}-R_{c} B_{1}+R_{c} B_{c}\right)\right) \\
\text { s.t. } & \forall\left\{v_{i}, v_{j}\right\} \in E_{c} \quad S_{i, j} \leq Y_{i} \\
& \forall\left\{v_{i}, v_{j}\right\} \in E_{c} \quad S_{i, j} \leq Y_{j} \\
& R_{1}=\sum_{v_{i} \in V_{R_{c}}} k_{i} Y_{i} \\
& B_{1}=\sum_{v_{j} \in V_{B_{c}}} k_{j} Y_{j} \\
& \forall v_{i} \in V_{R_{c}}, \forall v_{j} \in V_{B_{c}} \quad W_{i, j} \geq Y_{i}+Y_{j}-1 \\
& Y_{g}=1, g=\arg \max \left\{k_{i}, \forall v_{i} \in V_{c}\right\} \\
& R_{1} \in \mathbb{R} \\
& B_{1} \in \mathbb{R} \\
& \forall\left\{v_{i}, v_{j}\right\} \in E_{c} \quad S_{i, j} \in \mathbb{R} \\
& \forall v_{i} \in V_{R_{c}}, \forall v_{j} \in V_{B_{c}} \quad W_{i, j} \in \mathbb{R}_{0}^{+} \\
& \forall v_{i} \in V_{c} \quad Y_{i} \in\{0,1\} .
\end{aligned}
$$

Compact Fortet linearization Starting from the $B O B_{1 a}$ model, it is possible to obtain a more compact formulation. First, the objective function (2.86) can be rewritten as:

$$
\frac{1}{m}\left(\sum_{\left\{v_{i}, v_{j}\right\} \in E_{c}}\left(2 S_{i, j}-Y_{i}-Y_{j}+1\right)-\frac{1}{m}\left(\sum_{v_{i} \in V_{R_{c}}} \sum_{v_{j} \in V_{B_{c}}} k_{i} k_{j}\left(2 W_{i, j}-Y_{i}-Y_{j}+1\right)\right)\right),
$$


where the first and second part of the objective function present a similar structure. Let $a_{i, j}$ be a parameter which is equal to 1 if there exists the edge $\left\{v_{i}, v_{j}\right\}$, and 0 otherwise. Moreover, let $H_{i, j}$ be the parameter defined as:

$$
\forall v_{i} \in V_{R_{c}}, \forall v_{j} \in V_{B_{c}} \quad H_{i, j}=a_{i, j}-\frac{k_{i} k_{j}}{m} .
$$

We can define a compact model $B O B_{1 b}$ as follows:

$$
\begin{array}{ll}
\max & \frac{1}{m} \sum_{v_{i} \in V_{R_{c}}} \sum_{v_{j} \in V_{B_{c}}} H_{i, j}\left(2 W_{i, j}-Y_{i}-Y_{j}+1\right) \\
\text { s.t. } & \forall v_{i} \in V_{R_{c}}, \forall v_{j} \in V_{B_{c}}: H_{i, j}<0 \quad W_{i, j} \geq 0 \\
& \forall v_{i} \in V_{R_{c}}, \forall v_{j} \in V_{B_{c}}: H_{i, j}<0 \quad W_{i, j} \geq Y_{i}+Y_{j}-1 \\
& \forall v_{i} \in V_{R_{c}}, \forall v_{j} \in V_{B_{c}}: H_{i, j}>0 \quad W_{i, j} \leq Y_{i} \\
& \forall v_{i} \in V_{R_{c}}, \forall v_{j} \in V_{B_{c}}: H_{i, j}>0 \quad W_{i, j} \leq Y_{j} \\
& Y_{g}=1, g=\arg \max \left\{k_{i}, \forall v_{i} \in V_{c}\right\} \\
& \forall v_{i} \in V_{R_{c}}, \forall v_{j} \in V_{B_{c}} \quad W_{i, j} \in \mathbb{R} \\
& \forall v_{i} \in V_{c} \quad Y_{i} \in\{0,1\} .
\end{array}
$$

\subsubsection{Square reformulation}

It is possible to reformulate the $B O B$ model to have only squares as nonlinearities in the objective function, and use CPLEX as done for the unipartite case. Consider this part of the objective function (2.76):

$$
2 R_{1} B_{1}-B_{c} R_{1}-R_{c} B_{1}
$$

First at all, we can write the last two terms as:

$$
\begin{aligned}
& B_{c} R_{1}=\left(B_{c}+R_{c}\right) R_{1}-R_{c} R_{1} \\
& R_{c} B_{1}=\left(B_{c}+R_{c}\right) B_{1}-B_{c} B_{1},
\end{aligned}
$$

therefore we can rewrite (2.98) as:

$$
2 R_{1} B_{1}-\left(B_{c}+R_{c}\right)\left(R_{1}+B_{1}\right)+B_{c} B_{1}+R_{c} R_{1}
$$

If we are able to introduce the terms $B_{1}{ }^{2}$ and $R_{1}{ }^{2}$, we can replace them and $2 R_{1} B_{1}$ with $\left(R_{1}+B_{1}\right)^{2}$. To do that, consider first the term $R_{c} R_{1}$. Using definitions (2.69) and (2.71), we can write it this way:

$$
R_{c} R_{1}=\sum_{v_{i} \in V_{R_{c}}} k_{i} \sum_{v_{j} \in V_{R_{c}}} k_{j} Y_{j}=\sum_{v_{i} \in V_{R_{c}}} k_{i}^{2} Y_{i}+\sum_{v_{i} \in V_{R_{c}}} \sum_{v_{j} \in V_{R_{c}}: j<i} k_{i} k_{j}\left(Y_{i}+Y_{j}\right) .
$$


As stated earlier, we are interested in adding the term $R_{1}{ }^{2}$. We can express it as:

$$
R_{1}^{2}=\sum_{v_{i} \in V_{R_{c}}} k_{i} Y_{i} \sum_{v_{j} \in V_{R_{c}}} k_{j} Y_{j}=\sum_{v_{i} \in V_{R_{c}}} k_{i}{ }^{2} Y_{i}+\sum_{v_{i} \in V_{R_{c}}} \sum_{v_{j} \in V_{R_{c}}: j<i} 2 k_{i} k_{j} Y_{i} Y_{j}
$$

where we use the fact that $Y_{i}=Y_{i}^{2}$, since $Y$ are binary variables. Comparing (2.99) and (2.100), it appears that we can write $R_{c} R_{1}$ in terms of $R_{1}^{2}$ in this way:

$$
\begin{aligned}
R_{1} R_{c} & =R_{1}^{2}-\sum_{v_{i} \in V_{R_{c}}} \sum_{v_{j} \in V_{R_{c}}: j<i} 2 k_{i} k_{j} Y_{i} Y_{j}+\sum_{v_{i} \in V_{R_{c}}} \sum_{v_{j} \in V_{R_{c}}: j<i} k_{i} k_{j}\left(Y_{i}+Y_{j}\right)= \\
& =R_{1}^{2}+\sum_{v_{i} \in V_{R_{c}}} \sum_{v_{j} \in V_{R_{c}}: j<i} k_{i} k_{j}\left(Y_{i}+Y_{j}-2 Y_{i} Y_{j}\right)=R_{1}^{2}+ \\
& +\sum_{v_{i} \in V_{R_{c}}} \sum_{v_{j} \in V_{R_{c}}: j<i} k_{i} k_{j}\left(Y_{i}-Y_{j}\right)^{2} .
\end{aligned}
$$

We can obtain a similar result for the term $B_{1} B_{c}$. More precisely, we can write:

$$
B_{1} B_{c}=B_{1}^{2}+\sum_{v_{i} \in V_{B_{c}}} \sum_{v_{j} \in V_{B_{c}}: j<i} k_{i} k_{j}\left(Y_{i}-Y_{j}\right)^{2}
$$

Finally, equation (2.98) can be reformulated as:

$$
\begin{aligned}
& 2 R_{1} B_{1}-\left(B_{c}+R_{c}\right)\left(R_{1}+B_{1}\right)+B_{c} B_{1}+R_{c} R_{1}=2 R_{1} B_{1}-\left(B_{c}+R_{c}\right)\left(R_{1}+B_{1}\right)+ \\
& +R_{1}^{2}+B_{1}^{2}+\sum_{v_{i} \in V_{R_{c}}} \sum_{v_{j} \in V_{R_{c}}: j<i} k_{i} k_{j}\left(Y_{i}-Y_{j}\right)^{2}+\sum_{v_{i} \in V_{B_{c}}} \sum_{v_{j} \in V_{B_{c}}: j<i} k_{i} k_{j}\left(Y_{i}-Y_{j}\right)^{2}= \\
& =\left(R_{1}+B_{1}\right)^{2}-\left(B_{c}+R_{c}\right)\left(R_{1}+B_{1}\right)+\sum_{v_{i} \in V_{R_{c}}} \sum_{v_{j} \in V_{R_{c}}: j<i} k_{i} k_{j}\left(Y_{i}-Y_{j}\right)^{2}+ \\
& +\sum_{v_{i} \in V_{B_{c}}} \sum_{v_{j} \in V_{B_{c}}: j<i} k_{i} k_{j}\left(Y_{i}-Y_{j}\right)^{2} .
\end{aligned}
$$

Using relationships (2.73)-(2.75), we can now write the model $B O B_{2}$. It is interesting to notice that the objective function is similar to the objective function (2.33) of the unipartite model $O B_{1}$. More precisely, the first part of the objective function is the same, as well as the terms $D_{1}$ and $-D_{1} D_{c}$. A first difference is the presence of the term $\frac{1}{m}$, instead of $\frac{1}{2 m}$ as in (2.33). Then, the term $\frac{D_{c}^{2}}{2}$ is replaced by $R_{c} B_{c}$, and there are the following additional terms:

$$
\sum_{v_{i} \in V_{R_{c}}} \sum_{v_{j} \in V_{R_{c}}: j<i} k_{i} k_{j}\left(Y_{i}-Y_{j}\right)^{2}+\sum_{v_{i} \in V_{B_{c}}} \sum_{v_{j} \in V_{B_{c}}: j<i} k_{i} k_{j}\left(Y_{i}-Y_{j}\right)^{2} .
$$

Considering the set of constraints of the $\mathrm{BOB}_{2}$ model, it is exactly the same as that of the model $O B_{1}$, thus underlying the strong relationship between these problems. The model $B O B_{2}$ is defined as follows: 


$$
\begin{aligned}
\max & \frac{1}{m}\left(\sum_{\left\{v_{i}, v_{j}\right\} \in E_{c}}\left(2 S_{i, j}-Y_{i}-Y_{j}\right)+\left|E_{c}\right|-\frac{1}{m}\left(D_{1}^{2}-D_{1} D_{c}+\right.\right. \\
& \left.\left.+\sum_{v_{i} \in V_{R_{c}}} \sum_{v_{j} \in V_{R_{c}}: j<i} k_{i} k_{j}\left(Y_{i}-Y_{j}\right)^{2}+\sum_{v_{i} \in V_{B_{c}}} \sum_{v_{j} \in V_{B_{c}}: j<i} k_{i} k_{j}\left(Y_{i}-Y_{j}\right)^{2}+R_{c} B_{c}\right)\right) \\
\text { s.t. } & \forall\left\{v_{i}, v_{j}\right\} \in E_{c} \quad S_{i, j} \leq Y_{j} \\
& \forall\left\{v_{i}, v_{j}\right\} \in E_{c} \quad S_{i, j} \leq Y_{i} \\
& D_{1}=\sum_{i=1}^{n} k_{i} Y_{i} \\
& Y_{g}=1, g=\arg \max \left\{k_{i}, \forall v_{i} \in V_{c}\right\} \\
& \forall\left\{v_{i}, v_{j}\right\} \in E_{c} \quad S_{i, j} \in \mathbb{R} \\
& D_{1} \in \mathbb{R} \\
& \forall v_{i} \in V_{c} \quad Y_{i} \in\{0,1\} .
\end{aligned}
$$

\subsubsection{Binary decomposition}

In order to linearize the term $R_{1} B_{1}$ we can employ the binary decomposition, similarly as done for unipartite case. We can express the variables $R_{1}$ and $B_{1}$ as:

$$
\begin{aligned}
& R_{1}=\sum_{v_{i} \in V_{R_{c}}} k_{i} Y_{i}=\sum_{h=0}^{t_{R}} 2^{h} a_{h} \\
& B_{1}=\sum_{v_{j} \in V_{B_{c}}} k_{j} Y_{j}=\sum_{l=0}^{t_{B}} 2^{l} b_{l},
\end{aligned}
$$

where $a_{h}$ and $b_{l}$ are binary variables, and the parameters $t_{R}$ and $t_{B}$ are defined, similarly to equation (2.45), as:

$$
\begin{aligned}
& 2^{t_{R}+1}-1 \geq R_{c} \quad \Rightarrow \quad t_{R}=\left\lceil\log _{2}\left(R_{c}+1\right)-1\right\rceil \\
& 2^{t_{B}+1}-1 \geq B_{c} \quad \Rightarrow \quad t_{B}=\left\lceil\log _{2}\left(B_{c}+1\right)-1\right\rceil .
\end{aligned}
$$

Using equations (2.109) and (2.110), we can express the product $R_{1} B_{1}$ in this way:

$$
R_{1} B_{1}=\sum_{h=0}^{t_{R}} 2^{h} a_{h} \sum_{l=0}^{t_{B}} 2^{l} b_{l}=\sum_{h=0}^{t_{R}} \sum_{l=0}^{t_{B}} 2^{l+h} a_{h} b_{l} .
$$


Finally, to linearize the products $a_{h} b_{l}$, we introduce the variables $R_{l, h}$; using again the Fortet linearization, $R_{l, h}$ are defined by these constraints:

$$
\begin{array}{ll}
\forall l \in\left\{0, \ldots, t_{B}\right\}, \forall h \in\left\{0, \ldots, t_{R}\right\} & R_{l, h} \geq 0 \\
\forall l \in\left\{0, \ldots, t_{B}\right\}, \forall h \in\left\{0, \ldots, t_{R}\right\} & R_{l, h} \geq a_{h}+b_{l}-1 .
\end{array}
$$

This leads to the model $B O B_{3}$ :

$$
\begin{aligned}
\max & \frac{1}{m}\left(\sum_{\left\{v_{i}, v_{j}\right\} \in E_{c}}\left(2 S_{i, j}-Y_{i}-Y_{j}\right)+\left|E_{c}\right|-\frac{1}{m}\left(2 \sum_{h=0}^{t_{R}} \sum_{l=0}^{t_{R}} R_{l, h}+\right.\right. \\
& \left.\left.-B_{c} R_{1}-R_{c} B_{1}+R_{c} B_{c}\right)\right)
\end{aligned}
$$

s.t. $\forall\left\{v_{i}, v_{j}\right\} \in E_{c} \quad S_{i, j} \leq Y_{i}$

$\forall\left\{v_{i}, v_{j}\right\} \in E_{c} \quad S_{i, j} \leq Y_{j}$

$R_{1}=\sum_{v_{i} \in V_{R_{c}}} k_{i} Y_{i}$

$B_{1}=\sum_{v_{j} \in V_{B_{c}}} k_{j} Y_{j}$

$R_{1}=\sum_{h=0}^{t_{R}} 2^{h} a_{h}$

$B_{1}=\sum_{l=0}^{t_{B}} 2^{l} b_{l}$

$\forall l \in\left\{0, \ldots, t_{B}\right\}, \forall h \in\left\{0, \ldots, t_{R}\right\} \quad R_{l, h} \geq a_{h}+b_{l}-1$

$Y_{g}=1, g=\arg \max \left\{k_{i}, \forall v_{i} \in V_{c}\right\}$

$R_{1} \in \mathbb{R}$

$B_{1} \in \mathbb{R}$

$\forall\left\{v_{i}, v_{j}\right\} \in E_{c} \quad S_{i, j} \in \mathbb{R}$

$\forall l \in\left\{0, \ldots, t_{B}\right\}, \forall h \in\left\{0, \ldots, t_{R}\right\} \quad R_{l, h} \in \mathbb{R}_{0}^{+}$

$\forall v_{i} \in V_{c} \quad Y_{i} \in\{0,1\}$

$\forall h \in\left\{0, \ldots, t_{R}\right\} \quad a_{h} \in\{0,1\}$

$\forall l \in\left\{0, \ldots, t_{B}\right\} \quad b_{l} \in\{0,1\}$.

\subsubsection{Numerical results}

We present the comparison of the numerical results obtained by the proposed reformulations on a $2.4 \mathrm{GHz}$ Intel Xeon CPU of a computer with 24 GB RAM running Linux and CPLEX 12.2, with the same configuration used for the MM problem (that is, MIP cutting plane generation disabled, and branching based on pseudo 
reduced costs). For bipartite case the instances described in the literature are not numerous. We selected some bipartite graphs, presented in Table 2.5, from the Pajek dataset [22]. The graphs are the following: Southern women, which represents the attendance at 14 social event by 18 Southern women; Supreme court voting represents the votes of 9 judges of the Supreme Court Justice on 26 important topics on the years 2000-2001. The two graphs "yes" and "no" represent respectively the votes yes and no of judges; social work is related to journals in the social work citation graph, but no more informations are provided by Pajek; Wafa-CEO represents Galaskiewicz's CEOs and clubs graph; divorces concerns 9 different causes of divorces in the 50 States of the United States; Hollywood movies represents the relationships between 40 song composers and 62 film producers, where an edge between the composer $A$ and the producer $B$ represents the fact that $A$ created the soundtrack for a film produced by $B$; Scotland interlocks lists the 136 multiple directors of the 108 largest joint stock companies in Scotland in 1904-1905; graph product represents relationships between authors and papers taken from the bibliography of the book [136] (note that this graph contains some disconnected components, since $m<n-1)$; network science has a structure similar to the previous graph, concerning coautorship between scientists.

\begin{tabular}{|c|c|c|c|c|c|}
\hline ID & Graph & $\mathrm{p}$ & $\mathrm{n}$ & $\mathrm{m}$ & Reference \\
\hline 1 & Southern women & 18 & 32 & 89 & {$[73]$} \\
\hline 2 & Supreme Court voting (yes) & 26 & 35 & 147 & {$[22]$} \\
\hline 3 & Supreme Court voting (no) & 26 & 35 & 86 & {$[22]$} \\
\hline 4 & social work & 18 & 36 & 99 & {$[22]$} \\
\hline 5 & Wafa - CEO & 26 & 41 & 98 & {$[245]$} \\
\hline 6 & divorces & 50 & 59 & 225 & {$[22]$} \\
\hline 7 & Hollywood movies & 62 & 102 & 192 & {$[85]$} \\
\hline 8 & Scotland interlocks & 108 & 244 & 358 & {$[224]$} \\
\hline 9 & graph product & 314 & 674 & 613 & {$[136]$} \\
\hline 10 & network science & 960 & 2549 & 2580 & {$[196]$} \\
\hline
\end{tabular}

Table 2.5: Informations about the graphs used in tests.

We now present the result obtained by the proposed formulations for the graphs presented above. If CPLEX was not able to solve an instance because of memory space overhead, we use the symbol "-".

The first comparison is between the two models using the Fortet linearization, namely $B O B_{1 a}$ and $B O B_{1 b}$. Results presented in Table 2.6 clearly show that the more compact formulation $B O B_{1 b}$ outperforms the other one.

In Table 2.7 we present the results obtained by the best Fortet linearization model $B O B_{1 b}$, the square formulation $B O B_{2}$ and the binary decomposition formulation $B O B_{3}$. It appears that the square formulation is the worst, even if it was the best choice for the MM problem. However, in that case there was only a 


\begin{tabular}{rrrrrrr}
\hline & & \multicolumn{3}{c}{$B O B_{1 a}$} & \multicolumn{2}{c}{$B O B_{1 b}$} \\
ID & $N_{c}$ & $Q$ & nodes & time & nodes & time \\
\hline 1 & 4 & 0.3409 & 437 & 0.30 & $\mathbf{7 2}$ & $\mathbf{0 . 1 9}$ \\
2 & 2 & 0.2704 & 154 & 0.19 & $\mathbf{1 0}$ & $\mathbf{0 . 0 9}$ \\
3 & 2 & 0.4538 & 45 & 0.14 & $\mathbf{6}$ & $\mathbf{0 . 0 7}$ \\
4 & 5 & 0.2883 & 2169 & 1.46 & $\mathbf{1 3 6 0}$ & $\mathbf{1 . 2 4}$ \\
5 & 4 & 0.3329 & 1963 & 1.25 & $\mathbf{2 7 6}$ & $\mathbf{0 . 4 4}$ \\
6 & 3 & 0.1876 & 1123 & 0.77 & $\mathbf{2 7}$ & $\mathbf{0 . 1 6}$ \\
7 & 8 & 0.4939 & 1223370 & 4440.04 & $\mathbf{4 0 7 1 0 4}$ & $\mathbf{3 0 3 8 . 0 6}$ \\
8 & - & - & - & - & - & - \\
9 & - & - & - & - & - & - \\
10 & - & - & - & - & - & - \\
\hline
\end{tabular}

Table 2.6: Comparison between the two Fortet reformulations.

square in the objective function (i.e., $D_{1}^{2}$ ), whilst in the $B O B_{2}$ model there are many other squares. For small instances, the best choice is the model $B O B_{1 b}$. For larger instances, the most suitable formulation is $B O B_{3}$, which employs the binary decomposition technique. In fact, the last three (largest) instances are solved only by $\mathrm{BOB}_{3}$.

\begin{tabular}{rrrrrrrrr}
\hline & & \multicolumn{4}{c}{$B O B_{1 b}$} & \multicolumn{2}{c}{$B O B_{2}$} & \multicolumn{2}{c}{$B O B_{3}$} \\
ID & $N_{c}$ & $Q$ & nodes & time & nodes & time & nodes & time \\
\hline 1 & 4 & 0.3409 & $\mathbf{7 2}$ & $\mathbf{0 . 1 9}$ & 3372 & 1.32 & 670 & 0.39 \\
2 & 2 & 0.2704 & $\mathbf{1 0}$ & $\mathbf{0 . 0 9}$ & 1074 & 1.39 & 618 & 0.43 \\
3 & 2 & 0.4538 & $\mathbf{6}$ & $\mathbf{0 . 0 7}$ & 132 & 0.14 & 183 & 0.19 \\
4 & 5 & 0.2883 & $\mathbf{1 3 6 0}$ & 1.24 & 67364 & 13.11 & 1854 & $\mathbf{0 . 9 3}$ \\
5 & 4 & 0.3329 & $\mathbf{2 7 6}$ & 0.44 & 117997 & 23.84 & 647 & $\mathbf{0 . 3 9}$ \\
6 & 3 & 0.1876 & $\mathbf{2 7}$ & $\mathbf{0 . 1 6}$ & 2497924 & 646.78 & 2521 & 2.12 \\
7 & 8 & 0.4939 & 407104 & 3038.06 & - & - & $\mathbf{3 8 9 1 0}$ & $\mathbf{5 . 2 6}$ \\
8 & 13 & 0.7153 & - & - & - & - & $\mathbf{3 7 9 3}$ & $\mathbf{5 . 8 1}$ \\
9 & 139 & 0.9363 & - & - & - & - & $\mathbf{7 1 9 2 7 5 4 8}$ & $\mathbf{1 5 4 5 0 . 4 0}$ \\
10 & 414 & 0.9696 & - & - & - & - & $\mathbf{9 1 9 1 7}$ & $\mathbf{3 8 . 4 9}$ \\
\hline
\end{tabular}

Table 2.7: Comparison between the different reformulations.

Finally, we compare the quality of this divisive heuristic with respect to other heuristics for the BMM problem. We report in Table 2.8 the results presented in [171]. The results are available only for three graphs of Table 2.5, namely the number 1 (Southern women), the number 8 (Scotland interlocks), and the number 10 (network science). However, in [171] tests are done with a version of the network science graph with 2579 edges, whereas our version has 2580 edges.

It turns out that the best results are obtained by algorithm LPAb+ on these instances. Our divisive heuristic obtains a worse result for the Southern women 


\begin{tabular}{lrrrrrr}
\hline & \multicolumn{2}{c}{ Graph ID 1 } & \multicolumn{2}{c}{ Graph ID 8 } & \multicolumn{2}{c}{ Graph ID 10 } \\
Algorithm & $N_{c}$ & $Q$ & $N_{c}$ & $Q$ & $N_{c}$ & $Q$ \\
\hline Adaptive-BRIM & 4 & $\mathbf{0 . 3 4 5 5}$ & 13 & 0.6861 & 107 & 0.8894 \\
LPA-BRIM & 4 & $\mathbf{0 . 3 4 5 5}$ & 17 & 0.7141 & 500 & 0.9363 \\
CNM & 3 & 0.3430 & 32 & 0.7008 & 414 & 0.9695 \\
MSG & 3 & 0.3411 & 30 & 0.7004 & 414 & 0.9687 \\
LPAb & 4 & 0.3192 & 60 & 0.5783 & 691 & 0.7808 \\
LPAb-MSG & 4 & $\mathbf{0 . 3 4 5 5}$ & 16 & $\mathbf{0 . 7 1 9 4}$ & 414 & 0.9695 \\
LPAb+ & 4 & $\mathbf{0 . 3 4 5 5}$ & 16 & $\mathbf{0 . 7 1 9 4}$ & 415 & $\mathbf{0 . 9 6 9 6}$ \\
Divisive & 4 & 0.3409 & 13 & 0.7153 & 414 & $\mathbf{0 . 9 6 9 6}$ \\
\hline
\end{tabular}

Table 2.8: Comparison between different heuristics for bipartite modularity maximization on three instances: 1 (Southern women), 8 (Scoltand interlocks) and 10 (network science).

graph. For Scotland interlocks, our modularity value is the second best one after the value obtained by both LPAb-MSG and LPAb+. Finally, for network science the value of modularity is equal to the one obtained by LPAb + . The results could be improved by applying the split and merge technique recently presented in [48]. The divisive heuristic is interesting in this context because it is a MP based approach. However, even if the computational times are larger than those required by LPAb+, the advantage is that it has not to be run many times as LPAb+.

\subsection{Clustering based on strong and almost-strong con- ditions}

In this section we present a contribution which is not related to modularity maximization and MP in general. As stated at the beginning of this chapter, one of the possible way to find communities in a graph consists of defining some conditions which must be satisfied by all the communities. ${ }^{1}$ Among the best known conditions, Radicchi et al. proposed the concept of community in the strong sense [210]:

Definition 2.3.1 (Community in the strong sense). A subset $S$ of vertices of a graph is called community in the strong sense if the number of neighbors of each vertex within $S$ is larger than the number of neighbors outside $S$.

Using the notation of indegree and outdegree presented in Section 2.1, we can express this as $\forall v_{i} \in S \quad k_{i}^{\text {in }}>k_{i}^{\text {out }}$, or equivalently, employing the adjacency matrix notation, $\forall v_{i} \in S \quad \sum_{v_{j} \in S} a_{i, j}>\sum_{v_{j} \in V \backslash S} a_{i, j}$. Note that the concept of defensive alliance, studied in graph theory (see the thesis [225] and references therein), is

\footnotetext{
${ }^{1}$ Note that in this section we employ the term community instead of cluster, to be consistent with the definitions proposed in the literature.
} 
very close to that of community in the strong sense and is obtained by substituting non-strict inequalities to strict ones.

We can also introduce the straightforward definition of partition in the strong sense:

Definition 2.3.2 (Partition in the strong sense). A partition in the strong sense consists only of communities in the strong sense.

However, the definition of strong community seems to be too stringent, in the sense that a strong partition can be expected to contain only few communities, thus resulting not informative. More precisely, the bigger problems are related to the degree 2 vertices: following Definition 2.3.1, the two neighbors of a vertex having degree 2 must belong to the same community, together with the vertex itself. If this degree 2 vertex connects two heterogeneous communities, they could be merged in a single, big community.

As a matter of fact, tests done with well-known graphs of literature support this hypothesis. Therefore we introduce the concept of almost-strong community [44]:

Definition 2.3.3 (Community in the almost-strong sense). A subset $S$ of vertices of a graph is called community in the almost-strong sense if the number of neighbors of each vertex within $S$ is larger than the number of neighbors outside $S$, except for the degree 2 vertices, where the number of neighbors within $S$ can be larger or equal to the number of neighbors outside $S$.

This means that all the vertices $v_{i}$ having degree 2 must satisfy the condition $k_{i}^{\text {in }} \geq k_{i}^{\text {out }}$, and all the other vertices $v_{t}$ must satisfy the strong condition $k_{t}^{\text {in }}>k_{t}^{\text {out }}$. In other words, if a degree 2 vertex $v_{i}$ has two neighbors $v_{j}$ and $v_{h}$, we have three possibilities:

1. $v_{i}$ and $v_{j}$ belong to the same community, and $v_{h}$ to a different one;

2. $v_{i}$ and $v_{h}$ belong to the same community, and $v_{j}$ to a different one;

3. $v_{i}, v_{j}$ and $v_{h}$ belong to the same community.

Note that the only case allowed by the definition of community in the strong sense is the third one. We can now define the partition in the almost-strong sense:

Definition 2.3.4 (Partition in the almost-strong sense). A partition in the almost-strong sense consists only of communities in the almost-strong sense.

It turns out that partitions in the almost-strong sense are more informative that partitions in the strong sense, since all partitions in the strong sense are special cases of partitions in the almost-strong sense. The following proposition formalizes this fact: 
Proposition 2.3.5 (Inclusion property). Let $P_{S}$ be the set of partitions in the strong sense found for a given graph $G=(V, E)$, and $P_{A}$ be the set of partitions in the almost-strong sense found for the same graph. Then, $P_{S} \subseteq P_{A}$.

Proof. The only difference between communities in strong and almost-strong sense is related to degree 2 vertices. From Definition 2.3.3, we have three possible ways to assign a degree 2 vertex and its neighbors to communities, but only one of them, namely the number 3 , is compatible with the strong community definition. Thus, $P_{S}$ is the set of partitions in the almost-strong sense where we always choice to put each degree 2 vertex and its neighbors in the same community.

Another interesting property of the almost-strong communities is that if we merge two of them, we obtain another valid almost-strong partition, as proved by the following proposition:

Proposition 2.3.6 (Almost-strong merging property). Let $P$ be a partition in the almost-strong sense found for a given graph $G=(V, E)$, consisting of the almost-strong communities $C_{1}, C_{2}, \ldots, C_{M}$. Let $P^{\prime}$ be the partition composed by all the communities of $P$ except for two of them, namely $C_{j}$ and $C_{k}$, which are replaced by a new community $C_{i}$ obtained by merging $C_{j}$ and $C_{k}$. Then, $P^{\prime}$ is a partition in the almost-strong sense.

Proof. Let $v_{t}$ be a vertex belonging to $C_{j}$ or $C_{k}$, and then, after the merging, to $C_{i}$. Let $k_{t}^{i n}$ and $k_{t}^{\text {out }}$ be respectively the indegree and outdegree of $v_{t}$ before the merging, and $k_{t^{\prime}}^{\text {in }}, k_{t^{\prime}}^{\text {out }}$ be the same quantities after the merging. The consequence of the merging is that the indegree of $v_{t}$ increases (or remains unchanged), whereas its outdegree decreases (or remains unchanged). More precisely, the indegree increases, and the outdegree decreases, if the vertex $v_{t}$ has some neighbors in the community that will be merged with its own one. If $v_{t}$ has degree 2 , the almost-strong condition for partition $P$ imposes that:

$$
k_{t}^{\text {in }} \geq k_{t}^{\text {out }} .
$$

It also holds, from the previous considerations:

$$
k_{t^{\prime}}^{\text {in }} \geq k_{t}^{\text {in }} \geq k_{t}^{\text {out }} \geq k_{t^{\prime}}^{\text {out }}
$$

Hence, the almost-strong condition for $v_{t}$ (i.e., $k_{t^{\prime}}^{\text {in }} \geq k_{t^{\prime}}^{\text {out }}$ ) is also verified after the merging. On the other hand, if $v_{t}$ has degree $\neq 2$, it holds that:

$$
k_{t}^{\text {in }}>k_{t}^{\text {out }} .
$$

Again, from the previous considerations, we have:

$$
k_{t^{\prime}}^{\text {in }} \geq k_{t}^{\text {in }}>k_{t}^{\text {out }} \geq k_{t^{\prime}}^{\text {out }} .
$$


Hence, the almost-strong condition for $v_{t}$ (i.e., $k_{t^{\prime}}^{\text {in }}>k_{t^{\prime}}^{\text {out }}$ ) also holds when the degree of $v_{t}$ is not 2 . Since all the vertices in the new community $C_{i}$ respect the almoststrong condition and all the other communities remain unchanged, the partition $P^{\prime}$ is also an almost-strong partition.

The same property also holds for the strong partitions, as proved in the following corollary:

Corollary 2.3.7 (Strong merging property). Proposition 2.3.6 is also valid for strong partitions.

Proof. From Proposition 2.3.5, the strong partitions are a subset of the almost-strong partitions. Thus, Proposition 2.3.6 is also valid if we consider strong partitions.

In the remainder of this section we introduce two MP model to describe the problem of finding respectively partitions in the strong and almost-strong sense. However, since these problems are too large to be solved efficiently, two algorithms to enumerate respectively the partitions in the strong and almost-strong sense are proposed, and then the results obtained with some well-known graphs of the literature are compared.

\subsubsection{Strong communities detection}

The problem of finding communities in the strong sense can be described by means of a MILP model. Given a graph, let $V$ be the set of $n$ vertices, $E$ the set of edges and $k_{i}$ the degree of the vertex $v_{i}$ (as in the rest of the chapter). Moreover, $P$ is the set $\{1, \ldots, n\}$ of indices of the communities (since we do not know how many communities there are, but an upper bound is $n$, this set has cardinality $n), C_{t}$ is the variable equals to 1 if the community $t$ contains at least one vertex and 0 otherwise, and $Z_{i, t}$ is the binary variable equals to 1 if the vertex $v_{i}$ is inside the community 
$C_{t}$. The MP model is the following:

$$
\begin{array}{ll}
\max & \sum_{t \in P} C_{t} \\
\text { s.t. } & \forall v_{i} \in V \quad \sum_{t \in P} Z_{i, t}=1 \\
& \forall t \in P, \forall v_{j} \in V \quad \sum_{\left\{v_{i}, v_{j}\right\} \in E} Z_{i, t} \geq Z_{j, t}\left(\left\lfloor\frac{k_{j}}{2}\right\rfloor+1\right) \\
& \forall t \in P \quad C_{t} \leq \sum_{v_{i} \in V} Z_{i, t} \\
& \forall t \in P: t<n \quad C_{t} \leq C_{t+1} \\
& \forall t \in P \quad C_{t} \leq 1 \\
& \forall t \in P \quad C_{t} \in \mathbb{R} \\
& \forall v_{i} \in V, \forall t \in P \quad Z_{i, t} \in\{0,1\}
\end{array}
$$

where the objective function (2.111) aims at maximizing the number of communities, the constraints (2.112) force each vertex to belong to only one community, the constraints (2.113) express the strong condition for the vertex $v_{j}$ belonging to the community $t$, the constraints (2.114) fix to 1 the variable $C_{t}$ if there is at least one vertex in the community $t, 0$ otherwise (this holds because these variables are maximized by the objective function). Note that the variables $C$ do not need to be defined as binary, but only smaller than or equal to 1 (see constraints (2.116)). The constraints (2.115) are SBCs used to impose that the communities non-empty are the ones having bigger index (these kind of lexicographic order SBCs are presented in detail in the next chapter, in Section 3.3.2). The main problem of this formulation is that there are $n^{2}$ binary variables $Z$. A formulation having $O\left(n^{2}\right)$ binary variables can be only solved for small instances. This is the reason why we design a specific algorithm to find partitions in the strong sense (actually, the definition of MP models to describe strong and almost-strong rules is a work in progress with Cafieri, Caporossi, Hansen, and Perron). In fact we present an algorithm, called SC (Strong Communities), to enumerate all the partitions in strong sense for a given graph $G=(V, E)$.

Note that this problem always has a solution, i.e., the trivial partition consisting in a single community containing all the vertices. The algorithm will make use of two types of labels associated with the vertices and the edges of $G$ respectively: label $l_{i}$ associated with vertex $v_{i}, i=1, \ldots, n$ (initially $l_{i}=i$ for all vertices, and at the current iteration the label of the vertex $v_{i}$ is equal to the smallest label of a vertex of the community to which $v_{i}$ belongs); the label $t_{i, j}$ associated with edge $\left\{v_{i}, v_{j}\right\}$ can take three values $(-1,0,1)$. It is equal to -1 if it has already been decided that the vertices $v_{i}$ and $v_{j}$ belong to different communities; it is equal to 1 if it has already been decided that vertices $v_{i}$ and $v_{j}$ belong to the same community. If no decision 
has been taken, $t_{i, j}=0$. The rules of this algorithm are the following:

- Rule 1 (pending edges): if the edge $\left\{v_{i}, v_{j}\right\}$ is a pending one, set its label $t_{i, j}$ to 1 and set both $l_{i}$ and $l_{j}$ to $\min \left(l_{i}, l_{j}\right)$. In words, both vertices of a pending edge must belong to the same community.

- Rule 2 (degree two vertices): if vertex $v_{i}$ has degree $k_{i}=2$, and its neighbors are $v_{j}$ and $v_{k}$, set $t_{i, j}=1, t_{i, k}=1$ and $l_{i}=l_{j}=l_{k}=\min \left(l_{i}, l_{j}, l_{k}\right)$. In words, if a vertex $v_{i}$ has degree 2 and neighbors $v_{j}$ and $v_{k}$, it follows from the strong condition that all three vertices $v_{i}, v_{j}, v_{k}$ must belong to the same community.

Note that the Rules 1 and 2 should be applied only once, at the beginning of the resolution. Moreover, the order of selection of the vertices for Rule 2 does not change the communities found (regardless of the labels for each community).

- Rule 3.a (positive transitivity): if $l_{i}=l_{j}$ and $t_{i, j}=0$, set $t_{i, j}=1$. In words, if two vertices $v_{i}$ and $v_{j}$ belong to the same community, and are joined by an edge which does not specify that, set the label of this edge as positive.

- Rule 3.b (negative transitivity): if $l_{i} \neq l_{j}$ and $t_{i, j}=-1$, set $t_{a, b}=-1 \forall\left\{v_{a}, v_{b}\right\}$ : $l_{a}=l_{i}, l_{b}=l_{j}$, and $t_{a, b}=0$. In words, if two vertices belong to different communities and are joined by a negative edge, set to -1 all the edges with label 0 joining two vertices of these communities.

- Rule 4.a (majority 1): if the majority of neighbors of the vertex $v_{i}$ belong to the same community and the label of the vertices belonging to this community is $l$, set $l_{i}=l=\min \left(l, l_{i}\right)$ and apply the positive transitivity Rule. In words, if half or more of the neighbors of $v_{i}$ have the same label $l$, the only way to satisfy the strict inequality of the strong condition is to add the vertex $v_{i}$ to the community where its vertices have label $l$.

- Rule 4.b (majority 2): if a vertex $v_{i}$ has degree $k_{i}=2 d$, and there are $d$ neighbors with label $l_{1}$ and $d$ neighbors with label $l_{2}$, set $l_{i}=\min \left(l_{1}, l_{2}\right)$, and for all the vertices $v_{k}$ with $l_{k}=l_{1}$ or $l_{k}=l_{2}$, set $l_{k}=l_{i}$. Then, apply the positive transitivity Rule. In other words, we merge the communities with labels $l_{1}$ and $l_{2}$, and we put in this new community the vertex $v_{i}$, too.

- Rule 4.c (majority 3): if the number of negative edges, i.e., edges labeled with -1 , incident with the vertex $v_{i}$ is equal to $\left\lceil\frac{k_{i}}{2}\right\rceil-1$, for all the neighbor vertices $v_{j}$ of $v_{i}$ having $t_{i, j}=0$, set $t_{i, j}=1$, and for all the vertices $v_{k}$ with $l_{k}=l_{j}$, set $l_{k}=l_{i}$. In words, when the number of negative edges incident to $v_{i}$ is almost the majority there is only one way to satisfy the strong condition at vertex $v_{i}$, i.e., set the label associated to all other incident edges to 1.

Rules 3 and 4 must be repeated as long as there is at least one change of label. 
- Rule 5 (branching): if no more labels of edges can be modified according to the previous rules, select an edge with label 0 which joins the two largest communities. Set the label of this edge to -1 (left branch), then set separately this label to 1 (right branch). The application of Rule 5 so generates always two subproblems of the current problem, corresponding respectively to label -1 and label 1 for the selected edge. The two subproblems are stored and the algorithm proceeds returning to Rule 3.a to process each of the stored subproblems (one at a time).

- Rule 6.a (no majority): if the number of negative edges incident with the vertex $v_{i}$ is larger than $\left\lceil\frac{k_{i}}{2}\right\rceil-1$, apply Rule 8 below.

- Rule 6.b (no coherent labels): if there exist two vertices $v_{i}$ and $v_{j}$ with $l_{i}=l_{j}$ and $t_{i, j}=-1$, apply Rule 8 below.

- Rule 7 (feasible solution): if all edges have a label -1 or 1 , store the corresponding partition, then apply Rule 8 .

- Rule 8 (backtracking): return to the latest application of the branching rule and consider the right hand-side branch as current subproblem.

\subsubsection{Almost-strong communities detection}

As done for partitions in the strong sense, this problem can be described as a MILP:

$$
\begin{array}{ll}
\max & \sum_{t \in P} C_{t} \\
\text { s.t. } & \forall v_{i} \in V \quad \sum_{t \in P} Z_{i, t}=1 \\
& \forall t \in P, \forall v_{j} \in V: k_{j} \neq 2 \sum_{\left\{v_{i}, v_{j}\right\} \in E} Z_{i, t} \geq Z_{j, t}\left(\left\lfloor\frac{k_{j}}{2}\right\rfloor+1\right) \\
& \forall t \in P, \forall v_{j} \in V: k_{j}=2 \sum_{\left\{v_{i}, v_{j}\right\} \in E} Z_{i, t} \geq Z_{j, t} \\
& \forall t \in P \quad C_{t} \leq \sum_{v_{i} \in V} Z_{i, t} \\
& \forall t \in P: t<n \quad C_{t} \leq C_{t+1} \\
& \forall t \in P \quad C_{t} \leq 1 \\
& \forall t \in P \quad C_{t} \in \mathbb{R} \\
& \forall v_{i} \in V, \forall t \in P \quad Z_{i, t} \in\{0,1\},
\end{array}
$$

where the difference is the modification of the strong conditions (2.113): these conditions continue to hold for vertices having degree different to 2 (see constraints (2.121)), but the almost-strong version is introduced for vertices having degree 2 
(see constraints (2.122)). Comparing the almost-strong formulations (2.119)-(2.127) with the strong formulation (2.111)-(2.118) we notice that the almost-strong formulation is a relaxation (using the terminology introduced in Section 1.3.1.3) of the strong one, since the constraint (2.113) is relaxed for the vertices having degree two. Hence, the feasible region can be larger and the optimal solution of the almost-strong formulation is an upper bound on the optimal solution of the strong formulation, since these are maximization problems. This is a "mathematical programming" explanation of the fact that the partitions in the almost-strong sense can have a larger number of communities, that is a consequence of Proposition 2.3.5. Again, this problem is hard to solve, and we propose an enumerative algorithm to solve it in the following.

In fact, in order to find all the partitions in almost-strong sense, we modify the algorithm presented in the previous section and we adapt it to the Definition 2.3.3. Rules of this algorithm, called ASC (Almost-Strong Communities) are the following:

- Rule 1 (pending edges): if the edge $\left\{v_{i}, v_{j}\right\}$ is a pending one, set its label $t_{i, j}$ to 1 and set both $l_{i}$ and $l_{j}$ to $\min \left(l_{i}, l_{j}\right)$. In words, both vertices of pending edge must belong to the same community.

Note that the Rule 1 should be applied only once, at the beginning of the resolution. Rule 2 of SC algorithm has been removed due to considerations presented after Definition 2.3.3.

- Rule 3.a (positive transitivity): if $l_{i}=l_{j}$ and $t_{i, j}=0$, set $t_{i, j}=1$. In words, if two vertices $v_{i}$ and $v_{j}$ belong to the same community, and are joined by an edge which does not specify that, set the label of this edge as positive.

- Rule 3.b (negative transitivity): if $l_{i} \neq l_{j}$ and $t_{i, j}=-1$, set $t_{a, b}=-1 \forall\left\{v_{a}, v_{b}\right\}$ : $l_{a}=l_{i}, l_{b}=l_{j}$, and $t_{a, b}=0$. In words, if two vertices belong to different communities and are joined by a negative edge, set to -1 all the edges with label 0 joining two vertices of these communities.

- Rule 4.a.1 (majority 1): if the majority of neighbors of the vertex $v_{i}$ with $k_{i} \neq 2$ belong to the same community and the label of the vertices belonging to this community is $l$, set $l_{i}=l=\min \left(l, l_{1}\right)$ and apply the positive transitivity Rule.

- Rule 4.a.2 (majority 1'): if both neighbors of the vertex $v_{i}$ with $k_{i}=2$ belong to the same community and the label of the vertices belonging to this community is $l$, set $l_{i}=l=\min \left(l, l_{1}\right)$ and apply the positive transitivity Rule.

- Rule 4.b (majority 2): if a vertex $v_{i}$ has degree $k_{i}=2 d \neq 2$, and there are $d$ neighbors with label $l_{1}$ and $d$ neighbors with label $l_{2}$, set $l_{i}=\min \left(l_{1}, l_{2}\right)$, and for all the vertices $v_{k}$ with $l_{k}=l_{1}$ or $l_{2}$, set $l_{k}=l_{i}$. Then, apply the positive 
transitivity Rule. In other words, merge the communities with labels $l_{1}$ and $l_{2}$, and put in this new community the vertex $v_{i}$, too.

- Rule 4.c.1 (majority 3): if the number of negative edges incident with the vertex $v_{i}$ with degree $k_{i} \neq 2$ is equal to $\left\lceil\frac{k_{i}}{2}\right\rceil-1$, for all the neighbor vertices $v_{j}$ of $v_{i}$ having $t_{i, j}=0$, set $t_{i, j}=1$, and for all the vertices $v_{k}$ with $l_{k}=l_{j}$, set $l_{k}=l_{i}$.

- Rule 4.c.2 (majority 3'): if the number of negative edges incident with the vertex $v_{i}$ with degree $k_{i}=2$ is equal to 1 , for all the neighbor vertices $v_{j}$ of $v_{i}$ having $t_{i, j}=0$, set $t_{i, j}=1$, and for all the vertices $v_{k}$ with $l_{k}=l_{j}$, set $l_{k}=l_{i}$.

Rules 3 and 4 must be repeated as long as there is at least one change of label.

- Rule 5 (branching): if no more labels of edges can be modified according to the previous rules, select an edge with label 0 which joins the two largest communities. Set the label of this edge to -1 (left branch). Then set separately this label to 1 (right branch), store the current subproblem, and return to Rule 3.a.

- Rule 6.a.1 (no majority): if the number of negative edges incident with a vertex $v_{i}$ with degree $k_{i} \neq 2$ is larger than $\left\lceil\frac{k_{i}}{2}\right\rceil-1$, apply Rule 8 below.

- Rule 6.a.2 (no majority'): if the number of negative edges incident with a vertex $v_{i}$ with degree $k_{i}=2$ is larger than 1 , apply Rule 8 below.

- Rule 6.b (no coherent labels): if there exist two vertices $v_{i}$ and $v_{j}$ with $l_{i}=l_{j}$ and $t_{i, j}=-1$, apply Rule 8 below.

- Rule 7 (feasible solution): if all edges have a label -1 or 1 , store the corresponding partition, then apply Rule 8 .

- Rule 8 (backtracking): return to the latest application of the branching rule and consider the right hand-side branch as current subproblem.

\subsubsection{Comparison between SC and ASC}

We compare the results obtained by the two algorithms SC and ASC for 4 graphs. The vertices belonging to the same community are represented with the same shape and color in the figures. Since the partitions in the almost-strong sense can be numerous, we only consider those with the largest number of communities. Note that the trivial partition with a single cluster containing all the vertices is found by both SC and ASC.

The first graph considered is Zachary's karate club [251]. It consists of 34 vertices associated to the members of a karate club, while edges represents friendship relationships between these members after a split due to a dispute between the karate club administrator and the instructor. 
The split observed by Zachary leads to the communities $C_{1}=\{1,2,3,4,5,6,7,8$, $10,11,12,13,14,17,18,20,22\}$ and $C_{2}=V \backslash C_{1}$. The SC algorithm finds only the trivial partition and the one presented in Figure 2.2(a), while ASC finds the partition presented in Figure 2.2(b), and other 22 partitions into two communities. In particular one of these partitions, obtained by merging the diamond and circle shaped communities (see Proposition 2.3.6), is the partition found by Zachary.

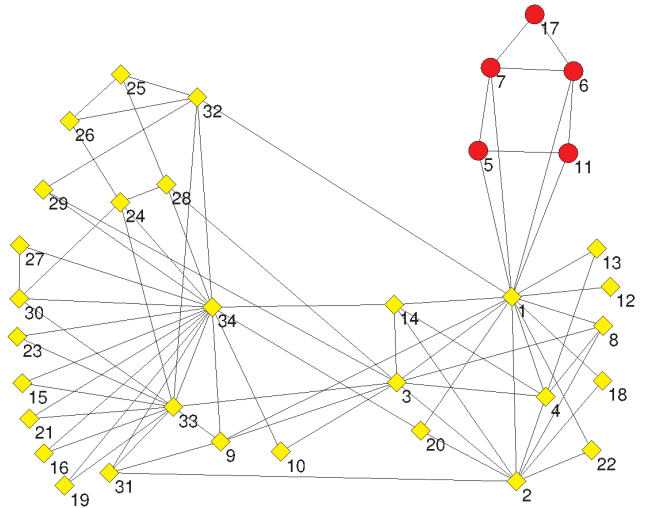

(a)

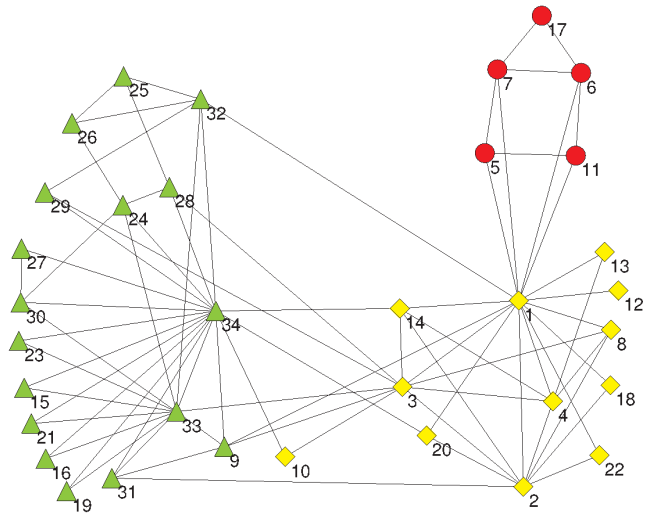

(b)

Figure 2.2: Partitions into strong and almost-strong communities obtained by algorithms SC and ASC respectively for Zachary karate club graph.

The second graph concerns informal communications within a sawmill on strike [189]. Vertices are associated with the 24 employees of a wood processing facility where a new management team proposes changes to the compensation package. The workers refuse and a strike follows. Facing a stalemate, the management asks a consultant to analyze the communications among the employees. Edges of the graph correspond to frequent discussions on the strike between pairs of colleagues. Two partitions into strong communities were obtained with the SC algorithm; one of them, represented in Figure 2.3(a), is composed of a first community $C_{1}=\{10,11,12,13\}$ corresponding to all Spanish-speaking employees, and another one representing 20 employees who are English-speaking. The ASC algorithm gives 20 partitions. A single one of them has 4 communities (see Figure 2.3(b)), and none had more. The small community with 4 Spanish-speaking employees remains the same. The second community of 20 employees is split into 3 communities: a first one corresponds to 9 English-speaking employees with age smaller than or equal to 30 . The second community with 9 employees and the third one with 2 employees correspond to older English-speaking workers. The partition of the 24 employees in three communities, i.e., Spanish-speaking, young English-speaking, and older English-speaking employees obtained by joining the two last communities corresponds exactly to the partition obtained by the consultant. As the strong conditions and the almost-strong conditions remain satisfied when communities are merged (due to Proposition 2.3.6 and Corollary 2.3.7), the ASC algorithm did also find the optimal three community 
partition. Detection of the small community with employees 16 and 21 may be interpreted in that these employees are less talkative, or less concerned by the strike, than most of the others.

It thus appears that algorithm SC recognizes well a small, almost isolated community but groups unduly the others. Algorithm ASC finds the optimal partition and it perhaps provides a little more information. Note the importance of vertex 15 having degree 2 .

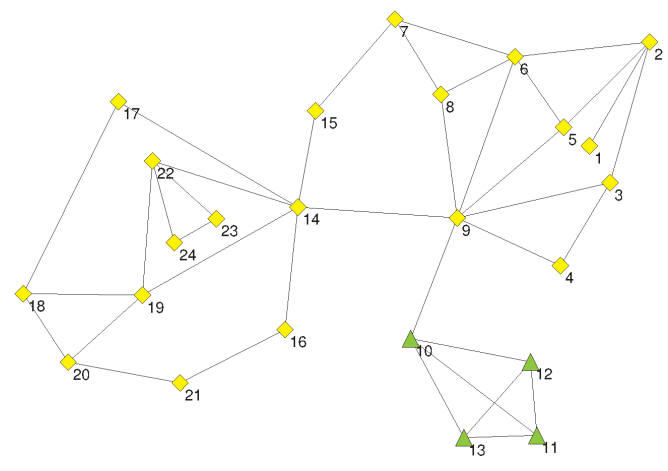

(a)

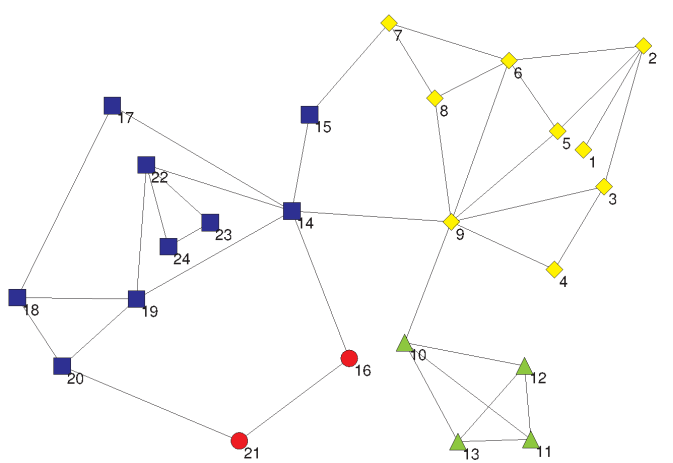

(b)

Figure 2.3: Partitions into strong and almost-strong communities obtained by algorithms SC and ASC respectively for the strike graph.

A next example is a directed graph representing a glossary of graphs and digraphs, which can be found in the Pajek repository [22]. An arc from $v_{i}$ to $v_{j}$ means that the concept associated with $v_{i}$ is used in the definition of $v_{j}$. We neglected orientation of arcs and considered only the largest connected component, which has 60 vertices and 114 edges. Applying algorithm SC only the trivial partition was found. Turning to algorithm ASC, many partitions were obtained, 5 of which have the largest number of communities, i.e., 6. The most intuitively appealing of them is presented on Figure 2.4. We next comment on these communities going from the smallest to the largest. The first community corresponds to two terms, i.e., \{complete, clique\}. They are clearly close, as a complete graph is a clique. The second community also has two terms, pertained to computer search, i.e., \{child, ordered tree\}. These two communities appeared unchanged in all 5 partitions into 6 communities. A third community contains 7 terms, i.e., \{decision tree, binary search tree, m-ary tree, rooted tree, offspring, level, height\}. All those terms correspond, as did those of community 2, to computer search. Community three is similar in the 4 other partitions into 6 communities except for that the term decision tree is assigned to another community. A fourth community contains 8 terms, i.e., \{diameter, distance, hamiltonian, walk, trail, path, acyclic graph, cycle\}. These terms correspond to concepts related to paths and cycles. A fifth community contains 17 terms, i.e., \{strongly connected, tournament, digraph, orientation, arc list, 


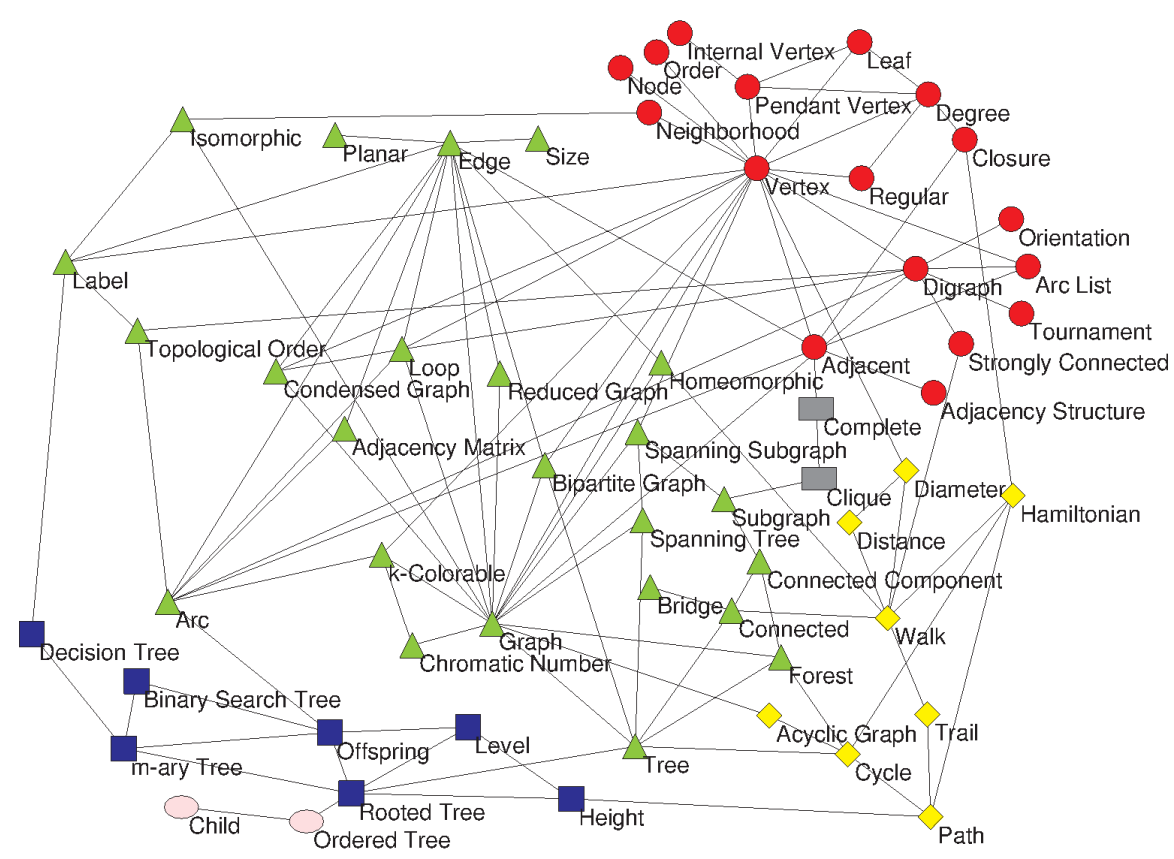

Figure 2.4: Partition into almost-strong communities obtained by algorithm ASC for the graph and digraph glossary graph.

neighborhood, node, order, internal vertex, vertex, pendant vertex, leaf, degree, regular, adjacency structure, adjacent, closure\}. It seems difficult to find a concept encompassing all of these terms. The five first, i.e., \{strongly connected, tournament, digraph, orientation, arc list\} correspond to oriented graphs. The remainder corresponds to vertices and adjacency. Note that this community contains several pairs of synonyms, i.e., $\{$ node $\}$ and $\{$ vertex $\}$, and $\{$ pendant vertex $\}$ and $\{$ leaf $\}$. The sixth community contains 24 terms, i.e., \{label, isomorphic, planar, edge, size, topological order, adjacency matrix, loop, reduced graph, condensed graph, homeomorphic, bipartite graph, spanning subgraph, subgraph, spanning tree, connected component, bridge, connected, forest, tree, graph, cromatic number, k-colorable, arc\}. This community appears to be less homogeneous than the others. Some concepts are related to edges, i.e., \{edge, loop, size, label\}. Others correspond to properties or families of graphs: \{isomorphic, homeomorphic, condensed graph, reduced graph, bipartite graph, spanning subgraph, spanning tree, subgraph, connected component, connected, bridge, tree, k-colorable, cromatic number, graph\}. Although this partition appears to be quite informative, it is not perfect, e.g., because \{forest\} and \{acyclic graph\} are synonyms but attributed to different communities. Or yet close terms such as $\{$ adjacency matrix $\}$ and adjacency structure $\}$ are also attributed to different communities.

A fourth example comes from the well-known paper on dolphins due to Lusseau et al. [175]. However, it does not concern the set of all 62 dolphins, but another graph giving the sociogram of the community for groups followed between 1995 and 2001. 
This graph has 40 vertices and 70 edges. When trying to find communities in the strong sense four partitions were obtained, one with three communities, represented on Figure 2.5(a), two obtained by merging pairs of adjacent communities, and the trivial partition.

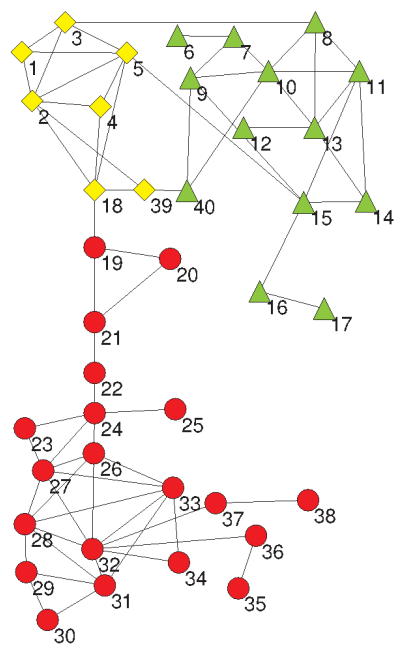

(a)

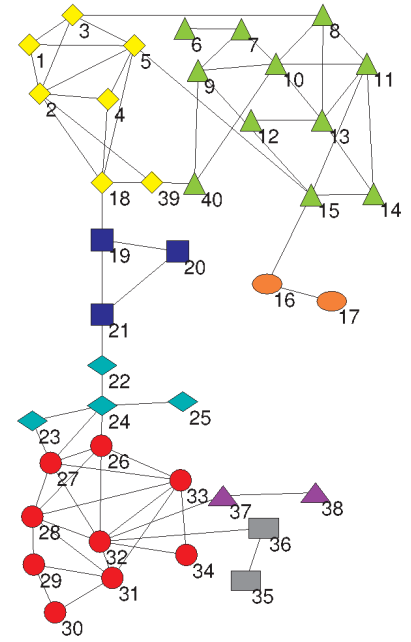

(b)

Figure 2.5: Partitions into strong and almost-strong communities obtained by algorithms $\mathrm{SC}$ and ASC respectively for the small dolphin graph.

Finding communities in the almost-strong sense gives a partition in eight communities, which is represented in Figure 2.5(b). It refines one of the communities by isolating a small two vertices community with one vertex of degree two. It also refines more drastically the largest community by isolating four subgraphs with two, two, three, and four entities. Each of these subgraphs contains a vertex of degree two. It appears clearly that this almost-strong partition is more informative than any other strong one.

\subsection{Conclusions}

In this chapter the problem of clustering in general and bipartite graphs is studied, and some techniques for finding good quality partitions are proposed. More precisely, we focus on modularity, and on some conditions which must be respected by each cluster.

In the fist part, we proposed some reformulations for the MP model used by a hierarchical divisive heuristic. Tests showed that the best results are obtained by means of a cMIQP model together with a SBC. As a matter of fact, we can employ CPLEX as solver, since the objective function is convex (the square variable has a negative coefficient in the objective function, and it is a maximization problem). We also presented some reformulations based on binary decomposition techniques for 
integer variables, which are not very efficient. However, this method has been introduced because it is the best choice for bipartite modularity maximization problem. Using general MINLP solvers, as well as applying directly Fortet inequalities was not considered, because too much time consuming.

We then considered the problem of maximizing bipartite modularity. First, we adapt the divisive heuristic for bipartite graphs. The original formulation used by the heuristic cannot be solved by CPLEX. Thus, starting from our knowledge on the previous case, we proposed some reformulations, i.e., a model presenting a convex objective function with squares, another one based on Fortet linearization (and starting from it, a more compact version), and a model which employs binary decompositions. In this case, the formulation with squares was the worst. This difference with respect to the unipartite case is due to the fact that for the bipartite case there are several squares in the objective function, whereas in the unipartite case there is only one square. For small instances the compact version of the model based on Fortet inequalities is the best, while the model based on the binary decomposition outperforms the former for larger instances. Indeed, the techniques employed in the unipartite case to obtain a more compact formulation and the SBC were employed as well.

Hence, for similar problems the best MP formulation can be different, and based on techniques which do not perform well in the other case. It is interesting to notice that the proposed reformulations are exact formulations of the original problem, because Fortet-based linearization for binary problem are exact. This is also showed in Chapter 4. Actually, we introduce a simple but effective SBC, thus obtaining a narrowing. However, the study of narrowings will be the subject of Chapter 3 .

In the last part of the thesis, we analyzed clustering from another point of view, that is by means of some rules defined for each cluster. Starting from the existing strong conditions, we relax them obtaining the almost-strong conditions, which give more informative partitions. Even if this part is not strictly related with reformulations, it represents another interesting approach for clustering problems. We first proposed MP formulations for the problems of finding partitions in the strong and almost-strong sense. Since the corresponding formulations are too large (in terms of binary variables) we proposed two enumerative algorithms to find these partitions. Adapting the terminology used for reformulations, we could say that the MP formulation associated to the almost-strong conditions is a relaxation of that one associated to the strong conditions, as it provides better results in terms of number of communities found, that is the objective function to maximize (as a relaxation provides usually an equal or better optimal solution with respect to the original problem) and the partitions obtained with the strong conditions are a subset of the partitions in the almost-strong sense (as a relaxation can have a larger feasible region with respect to the original problem). The study of relaxations is the topic of Chapter 4 . 


\section{Part II}

\section{An application of narrowings}



Circle packing in a square is a well-known problem in mathematics, with several applications. It is possible to describe it by means of MP, obtaining a NLP problem. However, due to its complexity, many approaches presented in the literature are heuristics. An interesting feature of the problem of packing equal circles in a square (PECS) is that it involves a high degree of symmetry, making it a good candidate for the application of narrowing reformulations. In this part of the thesis we first present the problem and some MP formulations to describe it. Then we characterize its symmetries, and we introduce some Symmetry Breaking Constraints (SBCs), which lead to narrowings, to break these symmetries, as well as some other constraints that improve the formulations. We compare the narrowings to the original formulation, showing that the former outperform the latter in terms of both computational time and size of the BB tree. We also propose a conjecture about the reduction of the range for some of the variables of the problem. This chapter is based on the papers [59-61,63,64]. The full proof of Theorem 3.4.2 is unpublished. 


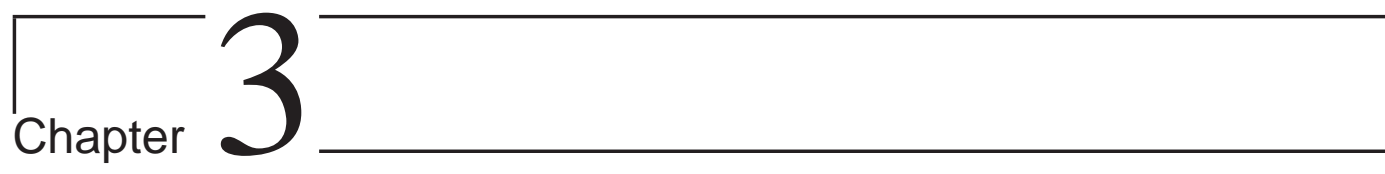

\section{Circle packing in a square}

Circle packing is a classical problem in mathematics [233,237]. Applications include cutting problems (cut out as many identical disks as possible from a piece of material) $[66,126,128]$, container loading (place as many identical cylindrical objects as possible into a container) $[99,103]$, and tree reforestation, where the aim is to plant trees (which grow approximately at the same speed) in a given region maximizing both their density and size. In this chapter we consider the problem of packing equal circles in a square having side-length 1. For an application-oriented survey see [52].

Circle packing in a square can be casted in form of optimization or decision problem. Moreover, there exist different but equivalent formulations for the problem itself: if an optimum for one of these is known, then we can easily find the optimal solutions for the others. Among the most known settings for the optimization version of this problem, we have the following:

Packing Equal Circles in a Square (PECS). Given an integer $n>0$, find the maximum common radius $r$ for $n$ non-overlapping circles arranged in the unit square.

Point Packing in a Square (PPS). Given an integer $n>0$, place $n$ points in the unit square such that their minimum pairwise distance $m$ is maximized.

Usually the PPS problem is stated in an alternative but equivalent way (details are provided in Section 3.1):

Point Packing in a Square (PPS). Given an integer $n>0$, place $n$ points in the unit square such that their squared minimum pairwise distance $\alpha$ is maximized.

The corresponding decision formulations of these problems are: 
DECISION VERSION OF PECS. Given an integer $n>0$ and a radius $r>0$, can $n$ circles of radius $r$ be packed in a unit square in such a way that the interiors of the circles have pairwise empty intersection?

DECISION VERSION OF PPS. Given an integer $n>0$ and a rational $\alpha \geq 0$, can $n$ points be determined in the unit square in such a way that their squared minimum pairwise distance is greater than or equal to $\alpha$ ?

In order to show the correspondence between PPS and PECS, here is a reduction from PPS to PECS: (a) every NO instance of the PPS problem is a NO instance of the PECS problem; (b) if a YES instance of the PPS problem is such that $r \geq \frac{\sqrt{\alpha}}{2+2 \sqrt{\alpha}}$ then it is also a YES instance of the PECS problem (the inequality can be verified easily by scaling the PPS configuration down so that it allows enough space to arrange circles wholly contained within the square); (c) otherwise, it is a NO instance of the PECS problem (Chapter 2 in [237]). Thus, given an instance of the PPS problem with its YES/NO decision, a YES/NO decision can be taken in constant time for the PECS problem. A similar transformation from PECS to PPS also holds. A graphical representation of the relationship between PECS and PPS is given in Figure 3.1.
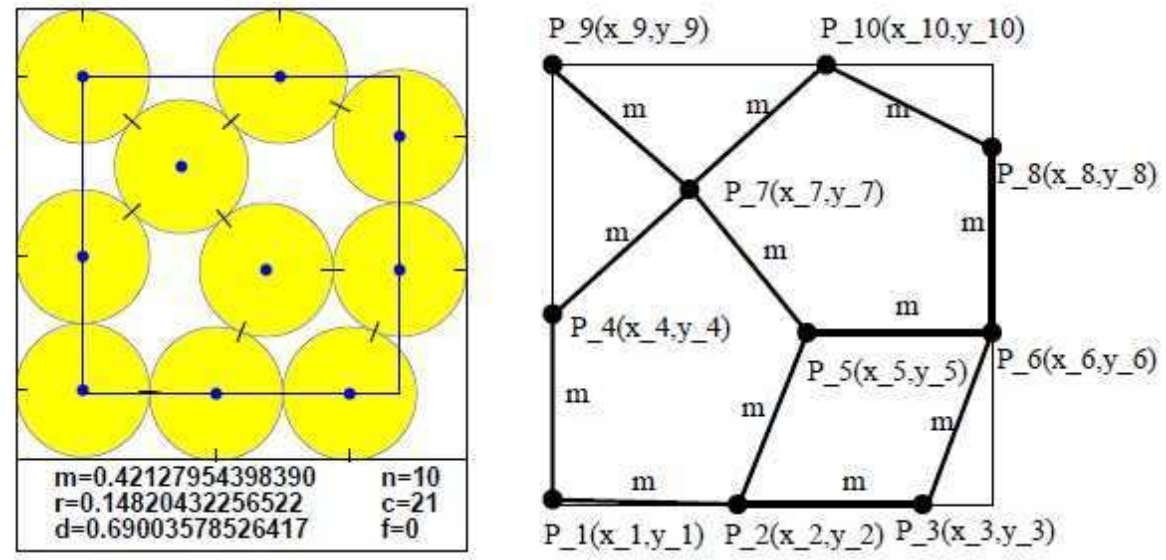

Figure 3.1: Optimal solutions of PECS and PPS for the instance where $n=10$. The picture is taken from [235].

The exact formula expressing the relationship existing between the radius $r_{n}$ of PECS and the distance $m_{n}$ of PPS when the number of circles (points) is $n$ is the following [237]:

$$
m_{n}=\frac{2 r_{n}}{1-2 r_{n}} .
$$

There exist also theoretical bounds on the optimal radius $r_{n}^{*}$ of PECS and the optimal distance $m_{n}^{*}$ of PPS, as reported in [237]:

Definition 3.0.1 (Bounds on the distance). For each $n \geq 2$ integer, it holds that:

$$
\sqrt{\frac{2}{\sqrt{3} n}} \leq m_{n}^{*} \leq \frac{1}{n-1}+\sqrt{\frac{1}{(n-1)^{2}}+\frac{2}{\sqrt{3}(n-1)}} .
$$


Definition 3.0.2 (Bound on the radius). For each $n \geq 2$ integer, it holds that:

$$
r_{n}^{*} \leq \min \left(\frac{1}{\sqrt{2 \sqrt{3} n+4(\lfloor\sqrt{n}\rfloor-2)(2-\sqrt{3})}}, \frac{1+\sqrt{1+\frac{2}{\sqrt{3}}(n-1)}}{2 n+2 \sqrt{1+\frac{2}{\sqrt{3}}(n-1)}}\right) .
$$

A different decision version of the problem, where radius is fixed and the side of the square is a parameter, is the following:

Given a rational $S>2$ and an integer $n>0$, can $n$ non-overlapping circles of radius 1 be arranged in a square of side $S$ ?

In this thesis we consider PECS and PPS (their optimization version). Their MP formulations are presented in details in Section 3.1. For more details about the existing formulations for this problem, see [237].

Complexity The PECS problem belongs to at least two classes of NP-hard problems: the Quadratically Constrained Quadratic Problem (QCQP) [243] and the Circle Packing Problem (CPP), where one is given a sequence of $n$ radii $r_{1}, \ldots, r_{n}$ and must decide whether $n$ circles with respective radii can fit in a unit square; the CPP was recently shown to be NP-hard [75]. The proof employs different radii and therefore does not seem applicable to PECS. Because the YES-certificates of PECS instances might involve irrational numbers, it is unclear whether PECS is in NP.

Let $\left(r_{1}, \ldots, r_{n}\right)$ be a YES instance of the CPP, and $\mathcal{C}=\left(\left(x_{i}, y_{i}\right) \mid i \leq n\right)$ be a certificate (i.e., the sequence of circle centers). The coin graph of $\mathcal{C}$ is an undirected graph $G=(V, E)$ such that $V=\{1, \ldots, n\}$ and for all $u, v \in V$ we have $(u, v) \in E$ if $\sqrt{\left(x_{u}-x_{v}\right)^{2}+\left(y_{u}-y_{v}\right)^{2}}=r_{u}+r_{v}$. It is known that a graph is a coin graph if and only if it is finite, simple, and planar [219]. Determining whether a given graph is a coin graph with unit edge lengths is NP-hard [40,82], but this does not take into account the PECS constraint that all circles should be contained in a square; furthermore, the instance for the PECS is simply a pair of numbers rather than a whole graph.

Many papers simply declare circle packing problems to be NP-hard (sometimes without stating any reference). As an example, [247] presents a heuristic for packing equal circles in an equilateral triangles: the authors state that the problem is NPhard and refer to $[101,129,130]$. The authors of [130] state in their introduction that:

For larger combinatorial [packing] problems these [simple] techniques become inefficient due to the vast number of possible solutions and the computation time grows exponentially. These problems are said to be NP-complete, 
a definitely questionable definition of NP-completeness; in the conclusion they also mention that "most packing problems are NP-complete". Garey and Johnson [101] only discuss set and bin packing problems, but not circle packing in the plane. The authors of [129] present polynomial-time approximation schemes for square covering, disc covering and square packing in a rectilinear region, but not disc packing; they cite $[98,138]$ for NP-completeness of the square packing problem. The authors of [98] exhibit a proof that packing equal boxes in a given region $R$ of the plane is NPcomplete (and they say that the proof can be extended to the case of equal discs). However, they work under the hypothesis that in $R$ there is only a finite number of box (disc) positions which might be required by an optimal packing. More precisely, they consider the graph $\mathcal{R}$ whose vertex set is $R$ and whose edge set includes pairs of points in $R$ which are closer than $2 r$, so that equal disc packings then correspond to stable sets in $\mathcal{R}$; but they assume $\mathcal{R}$ to be finite, which does not seem to be the case if $R$ is the unit square as in the PECS. In his NP-completeness column [138], Johnson reports the results of [98] as packing equal squares in a rectilinear polygon such that the squares are parallel to the axes, but omits to mention the disc packing result. In summary, to the best of our knowledge, there is no proof in the literature that offers a polynomial reduction from an NP-hard problem to PECS.

Related work Many different approaches were proposed to solve the PECS problem (or its equivalent formulation PPS), stemming from global optimization and geometry. The classical formulation of the PECS problem is as a QCQP $[173,178$, $209,236]$, but it can also be formulated as a d.c. (i.e., difference of convex functions) program [131]. A geometric BB method is introduced in [173], together with some characterizations of optimal solutions which are recalled later. An interval BB described in [237] is used to find guaranteed optimal packings whilst verifying floating point computations.

However, it should be remarked that most of the existing approaches are heuristics. A method coming from a physical interpretation of the problem is the minimization of energy function, where the circle centers are considered as electrical charges repulsing each other: if the distance between two points increases, the energy decreases $[201,237]$. In the billiard simulation method each circle is a ball with radius, speed, and direction; then the radius is increased until the structure of the packing becomes fixed [110]. A similar idea is used in the Pulsating Disk Shaking (PSD) algorithm [237]. The perturbation method tries to find good solutions for the PPS problem by moving the points in the square up, down, left or right; how much the points can be moved is determined by a parameter, and its value decreases during the process. After that, the position of a point is updated if the distance between the point and the neighbors increases [32]. TAMSASS-PECS algorithm combines both the Threshold Accepting method (TA) (where, as in the Simulated Annealing, a new solution is accepted if it decreases the quality of the current solution 
less than a given threshold), and a Modified version of the Single Agent Stochastic Search (MSASS) for the PECS problem [51,237]. Another approach based on a physical interpretation consists in the simulation of the movement of smooth elastic discs in a container [247]. In [133], a formulation-based multi-start heuristic with a combinatorial element (circles get moved to the largest vacant area of the current configuration before calling a local optimization procedure) is proposed for the PECS problem. Monotonic basin hopping heuristics have been proposed for packing equal and unequal circles in a square [3] and in a containing circle [112].

Another approach consists in finding a relationship between the number of circles and the structure of the packings (patterns): if these patterns can be found, it is easy to divide some packings into classes and thus to determine the coordinates of the centers of the circles; some experiments in this direction were performed in [110,201].

It is also possible to describe the structure of the optimal packing by means of a quadratical system of equations. After some manipulation, the problem can be reformulated as the solution of a polynomial, where the smallest positive root is the optimal solution for the PPS problem [235,237].

For more details, we refer to the book [237] and the surveys [127,236]. A very interesting work for a related problem, namely packing circles in a circle, where some valid inequalities involving the radius of circles and the coordinates of their centers are derived from real variable theory, complex variable theory and functional analysis is presented in [74].

The rest of the chapter is organized as follows: in Section 3.1 the MP formulations of PECS and PPS are presented. In Section 3.2 the symmetry structure of the problem is analyzed, and the resulting information is employed in Section 3.3 to derive some SBCs thus obtaining narrowing reformulations. After that, in Section 3.4 additional constraints which help to tighten the formulation are derived. Then, in Section 3.5 a conjecture about the reduction of the range for some of the variables of the problem is proposed. Finally, Section 3.6 presents the conclusions.

\subsection{Mathematical programming formulations}

We employ the following MP formulation for PECS:

$$
\begin{array}{cl}
\max & r \\
\text { s.t. } & \forall i<j \leq n \quad\left(x_{i}-x_{j}\right)^{2}+\left(y_{i}-y_{j}\right)^{2} \geq 4 r^{2} \\
& \forall i \leq n \quad x_{i} \in[r, 1-r] \\
& \forall i \leq n \quad y_{i} \in[r, 1-r] \\
& r \in \mathbb{R}_{0}^{+} .
\end{array}
$$


The objective function (3.1) aims to maximize the radius $r$; the distance constraints (3.2) make sure the circle interiors are pairwise disjoint; the constraints (3.3)-(3.4) make sure the circles are within the square.

The PECS formulation given above is a nonconvex NLP problem. The only nonconvexities are given by the reverse convex constraints (3.2). A simple multistart approach where a local NLP solver (such as SNOPT [104]) is deployed from a variety of randomly chosen starting points can convince that the PECS formulation has several different local optima.

Concerning PPS, it can be formulated as follows:

$$
\begin{aligned}
\max & \alpha \\
\text { s.t. } & \forall i<j \leq n \quad\left(x_{i}-x_{j}\right)^{2}+\left(y_{i}-y_{j}\right)^{2} \geq \alpha \\
& \forall i \leq n \quad x_{i} \in[0,1] \\
& \forall i \leq n \quad y_{i} \in[0,1] \\
& \alpha \in \mathbb{R}_{0}^{+},
\end{aligned}
$$

where, with respect to the PECS formulation, $\alpha=4 r^{2}$. Note that the same model having the distance $m$ as variable, and not its square $\alpha$ (i.e., the objective function is $m$ and the right hand side of constraint (3.7) is $m^{2}$ ) represents the first model of the PPS problem as defined in the beginning of the chapter. Since $\alpha=m^{2}$, we are actually maximizing $m^{2}$ in the PPS formulation (3.6)-(3.10). However, due to the nonnegativity of the distance $m$, this is equivalent to maximize $m$.

Although PECS and PPS are equivalent, the corresponding formulations are not. Specifically, the PECS formulation involves both $r$ and $r^{2}$, whereas the PPS formulation only involves a linear term $\alpha$ which replaces $4 r^{2}$ (given an optimal $\alpha$, the corresponding $r$ can be recovered in constant time). This formulation difference has an impact on sBB performance with implementations such as Couenne [26]: Table 3.1 shows that there is no clear efficiency domination on a per-instance basis. The cumulative CPU time and node count of the PECS formulation, however, are lower than their PPS counterparts. In the rest of the chapter, we shall employ the PECS formulation (3.1)-(3.5). PPS is employed in Section 3.5 when introducing a conjecture, for which the PECS formulation would provide a more complicated explanation.

\subsection{Detection of symmetries for circle packing}

The PECS problem has solution symmetries that stem from the geometry of the configurations (rotations and reflections of the square), as well as from the formulation itself (permutations of axes labels and point indices). The sBB tree is a rooted plane binary tree whose leaves contain globally optimal solutions (or rather, 


\begin{tabular}{rrrrr}
\hline & \multicolumn{2}{c}{ PECS } & \multicolumn{2}{c}{ PPS } \\
$n$ & CPU & nodes & CPU & nodes \\
\hline 2 & 0.03 & 0 & 0.04 & 0 \\
3 & 0.06 & 0 & 0.07 & 0 \\
4 & 0.12 & 0 & 0.10 & 0 \\
5 & 0.19 & 2 & 0.20 & 2 \\
6 & 14.30 & 94 & 3.18 & 220 \\
7 & 17.11 & 614 & 9.77 & 2360 \\
8 & 57.25 & 6952 & 41.94 & 9160 \\
9 & 553.62 & 69172 & 1334.82 & 339804 \\
\hline
\end{tabular}

Table 3.1: Comparing sBB on PECS and on PPS.

$\varepsilon$-approximations thereof). Intuitively, a formulation with fewer optimal solutions yield fewer leaves, smaller sBB trees, and faster convergence. If a set of different global optima can be obtained by symmetry from just one global optimum, we should aim to only keep one sBB branch leading to a single optimum, whilst discarding the other (symmetric) branches. One way to do this, that is the way we shall follow in this chapter, consists in reformulating the PECS formulation so that some symmetric solutions become infeasible. In other words, we adjoin some constraints to the formulation which are feasible with at least one global optimum, but might make several symmetric optima infeasible. Such constraints are called Symmetry Breaking Constraints (SBC) [158] (also called Static Symmetry Breaking Inequalities (SSBI) $[59,179]$ ), and the corresponding reformulation is a narrowing. An intuitive idea about the effect of SBCs on the sBB tree is provided in Figure 3.2.

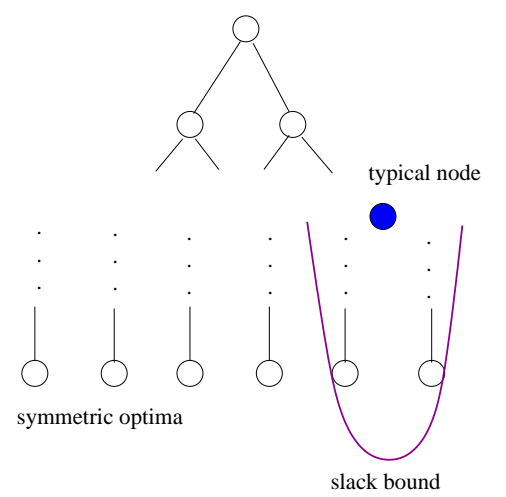

(a)

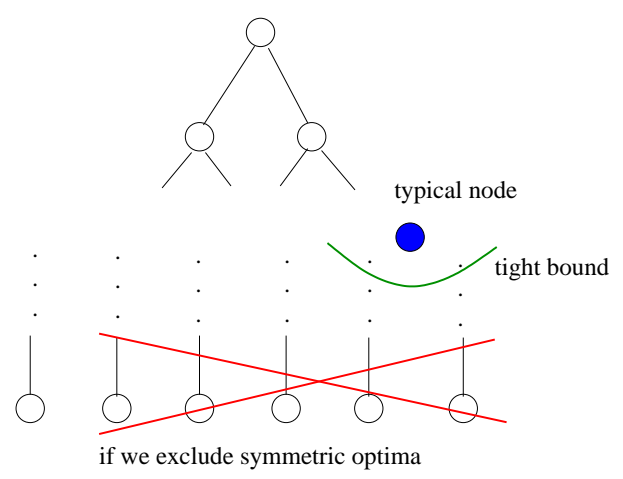

(b)

Figure 3.2: Trees of sBB associated to a formulation with (a) and without (b) SBCs.

Another motivation for studying SBCs is based on the empirical observation that good solutions for the PECS problem are found earlier when using the SBC-based narrowing. As a matter of fact, considering the description of sBB presented in Section 1.2.2.5, the incumbent is found by the local NLP solver in Step 4, and this means that the narrowing somehow "eases" local ascent towards good optima. More- 
over, the sBB applied to the proposed narrowing tightens the bound in Step 2 more effectively and thus solves the problem in less CPU time. In order to understand the reasons for this behavior, we shall introduce the linear relaxation employed by most sBB solvers, and constructed automatically from the problem formulation. The main steps are the following:

- replace all nonlinear terms $T(x, y)$ by an added variable $w_{T}$;

- compute lower and upper linear bounding functions $\breve{T}(x, y), \hat{T}(x, y)$ to $T(x, y)$ on the node box $B^{a}$;

- adjoin constraints $\check{T}(x, y) \leq w_{T} \leq \hat{T}(x, y)$ to the formulation.

In the case of the PECS formulation, the distance constraints (3.2) are the only ones that need to be relaxed, as they are the only nonconvex ones. The relaxation we obtain for the PECS formulation at the root node (where $B=[0,1]^{2 n+1}$ ) is:

$$
\begin{array}{cl}
\max & r \\
\text { s.t. } & \forall i<j \leq n \quad\left(X_{i}+X_{j}-2 W_{i j}\right)+\left(Y_{i}+Y_{j}-2 Z_{i j}\right) \geq 4 R \\
& \forall i<j \leq n \quad W_{i j} \leq \min \left\{x_{i}, x_{j}\right\} \\
& \forall i<j \leq n \quad W_{i j} \geq \max \left\{0, x_{i}+x_{j}-1\right\} \\
& \forall i<j \leq n \quad Z_{i j} \leq \min \left\{y_{i}, y_{j}\right\} \\
& \forall i<j \leq n \quad Z_{i j} \geq \max \left\{0, y_{i}+y_{j}-1\right\} \\
& \forall i \leq n \quad x_{i} \in[r, 1-r] \\
& \forall i \leq n \quad y_{i} \in[r, 1-r] \\
& \forall i \leq n \quad X_{i} \in\left[0, x_{i}\right] \\
& \forall i \leq n \quad Y_{i} \in\left[0, y_{i}\right] \\
& R \in[0, r] \\
& \forall i<j \leq n \quad W_{i j} \in \mathbb{R} \\
& \forall i<j \leq n \quad Z_{i j} \in \mathbb{R} \\
& r \in \mathbb{R}_{0}^{+},
\end{array}
$$

where, for each $i \leq n, X_{i} \in\left[0, x_{i}\right]$ are lower/upper bounding relaxations of $X_{i}=x_{i}^{2}$ on $x_{i} \in[0,1]$ (the same holds for $Y_{i}$ and $R$ ), and for all $i<j \leq n$ constraints (3.13)-(3.14) are lower and upper bounding relaxations for $x_{i} x_{j}$ on $[0,1] \times[0,1]$ (the same holds for $y_{i} y_{j}$ in constraints (3.15)-(3.16)).

Proposition 3.2.1. All optimal solutions of the PECS relaxation (3.11)-(3.24) have $\forall i \leq n \quad x_{i}=y_{i}=r=\frac{1}{2}$.

Proof. First, $r=\frac{1}{2}$ is the globally maximal value of the PECS relaxation, as any larger value would make (3.17)-(3.18) infeasible. Secondly, by (3.17)-(3.18), $r=\frac{1}{2}$ 
implies $x_{i}=y_{i}=\frac{1}{2}$ for all $i \leq n$. Any value of $W, Z, R$ in $\left[0, \frac{1}{2}\right]$ consistent with (3.12) (e.g., $W=Z=R=0$ ) yields a feasible solution with maximum objective function value.

Although the situation changes at lower level nodes, relaxations yielding $x_{i}=y_{i}$ for several values of $i$ is typical for several high-level nodes. We also remark that Proposition 3.2.1 also holds for the problem of packing equal hyperspheres in a unit hyperbox in $\mathbb{R}^{K}$.

Consider now the PECS instance with $n=2$ : since the root node relaxation solution has all components set to $\frac{1}{2}$, at Step 4 of the sBB procedure described in Section 1.2.2.5 the local NLP solver will use the central point of the square as a starting point to perform local descent from. Since there are four symmetric optima at exactly the same distance from the starting point, the local solution algorithm will have to consider four different ascent vectors (shown as the arrows in Figure 3.3) whose sum is the zero vector, making the starting point either a local maximum or a saddle. Adjoining the SBC $x_{1} \leq x_{2}$, for example, and assuming circle 1 is filled in Figure 3.3, would make the two leftmost configurations infeasible. This will make the sum of the ascent vectors nonzero, thereby easing the task of the local NLP solver. The benefits brought by SBCs to local NLP solvers will be further discussed
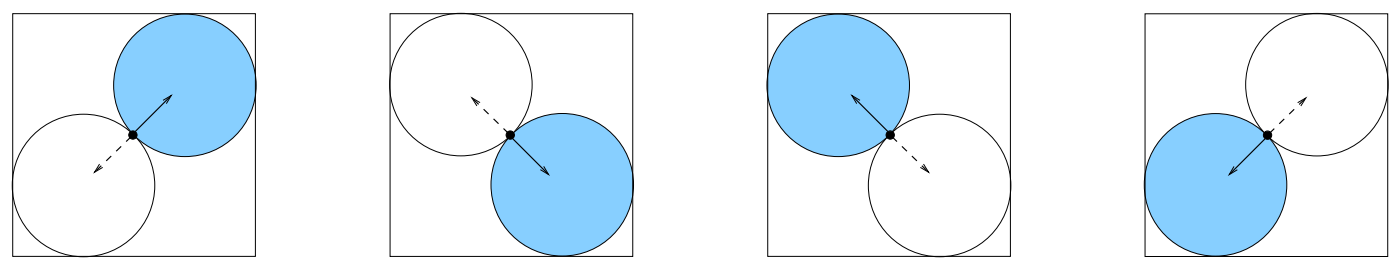

Figure 3.3: Four symmetric optima with $n=2$ : the sum of the four ascent directions from the central starting point towards the four optima is zero, both for solid and for dashed coordinates. If the two leftmost optima are infeasible (e.g., by means of the constraint $x_{1} \leq x_{2}$ ) the sum of the ascent directions becomes nonzero: positive (for the dashed coordinates) and negative (for the solid coordinates).

in Section 3.3.2.

From these considerations it appears that symmetries play an important role in the solution process of the PECS problem by means of sBB. Hence, it is crucial to characterize the symmetries of the PECS problem. To this aim, we introduce in the following a method to obtain automatically informations about some of the symmetries of a general MINLP problem. Using this method, we conjecture the symmetric structure of PECS, and then we prove this conjecture. This makes possible to derive some SBCs to adjoin to the MP model, obtaining narrowings. These constraints are presented in Section 3.3. Since the automatic symmetries detection method is based on concepts arising in group theory, some basic definitions and notation are provided in the next section. 


\subsubsection{Definitions and notation}

For $n \in \mathbb{N}$ we let $S_{n}$ be the symmetric group of order $n$ (i.e., the group of all permutations of $n$ symbols) and $C_{n}$ be the cyclic group of order $n$ (i.e., the group of rotations of a regular $n$-polygon). For a subset $N \subseteq\{1, \ldots, n\}$ we let $\operatorname{Sym}(N)$ be the symmetric group on the symbols of $N$. Consider a group $G$ and a set $X$. The action of $G$ on $X$ corresponds to the application of a permutation $g \in G$ to an element $x \in X$, and the result is indicated as $g x$. For $g \in G, x \in X$, we let $G x=\{g x \mid g \in G\}$ be the orbit of $x$ in $G$. For a subset $Y \subseteq X$ we let $\operatorname{stab}(Y, G)$, the setwise stabilizer of $Y$ in $G$, be the largest subgroup $H \leq G$ such that $H Y=Y$ (i.e., $h y \in Y$ for all $h \in H, y \in Y)$. In other words $\operatorname{stab}(Y, G)$ is the largest subgroup of $Y$ which maps an element of $X$ into another element of $X$. Let $D=(V, A)$ be a directed graph. An automorphism of $D$ is a permutation $\pi \in \operatorname{Sym}(V)$ such that $\forall(u, v) \in A(\pi(u), \pi(v)) \in A$. If $D$ has no cycles then it is a Directed Acyclic Graph (DAG).

\subsubsection{Automatic symmetry detection}

In this section we briefly present a method for computing MP symmetries automatically; conceptually, it is the same as in [158] and similar to [212] but the formal presentation is different. Consider a MINLP $P$ defined as:

$$
\begin{array}{cl}
\min & f(x) \\
\text { s.t. } & g(x) \leq 0 \\
& x \in X,
\end{array}
$$

where $f: \mathbb{R}^{n} \rightarrow \mathbb{R}, g: \mathbb{R}^{n} \rightarrow \mathbb{R}^{m}, x \in \mathbb{R}^{n}$, and $X \subseteq \mathbb{R}^{n}$ is a set which might include variable ranges $x^{L} \leq x \leq x^{U}$ as well as integrality constraints on a subset of variables $\left\{x_{i} \mid i \in I\right\}$ for some $I \subseteq\{1, \ldots, n\}$. Let $\mathcal{G}(P)$ be the set of global optima of $P$ and $\mathcal{F}(P)$ be its feasible region. We define the action of $S_{n}$ on $\mathbb{R}^{n}$ as follows: $\forall \pi \in S_{n}, x \in \mathbb{R}^{n}$ let $\pi\left(x_{1}, \ldots, x_{n}\right)=\left(x_{\pi^{-1}(1)}, \ldots, x_{\pi^{-1}(n)}\right)$ so that, for example, $(1,2,3)\left(x_{1}, x_{2}, x_{3}\right)=\left(x_{3}, x_{1}, x_{2}\right)$. The group $G_{P}^{*}=\operatorname{stab}\left(\mathcal{G}(P), S_{n}\right)$ is called the solution group of $P$. The solution group is the largest subgroup of $S_{n}$ which maps every global optimum into another global optimum. Since $G_{P}^{*}$ depends on $\mathcal{G}(P)$ it cannot, in general, be found before the solution process. We therefore try to find subgroups of $G_{P}^{*}$. In particular, we consider the subgroup of $G_{P}^{*}$ consisting of all variable permutations which "fix the formulation" of $P$. For $\pi \in S_{n}$ and $\sigma \in S_{m}$ we 
define $\sigma P \pi$ to be the following MINLP problem:

$$
\begin{array}{cl}
\min & f(\pi x) \\
\text { s.t. } & \sigma g(\pi x) \leq 0 \\
& \pi x \in X,
\end{array}
$$

where $\sigma$ acts on $g=\left(g_{1}, \ldots, g_{m}\right)$ by $\sigma g=\left(g_{\sigma^{-1}(1)}, \ldots, g_{\sigma^{-1}(m)}\right)$. Consider the group $\bar{G}_{P}=\left\{\pi \in S_{n} \mid \exists \sigma \in S_{m}(\sigma P \pi)=P\right\}$, that is the group of permutations $\pi$ on the variables of the problem for which it exists a permutation $\sigma$ on the constraints such that the problems (3.25)-(3.27) is the same as (3.28)-(3.30). Whenever $P$ is a MILP problem, $\bar{G}_{P}$ is called the LP relaxation group [179]. Unfortunately, for general MINLPs, determining whether $\forall x \in \operatorname{dom}(f) f(\pi x)=f(x)$ and $\forall x \in$ $\operatorname{dom}(g) \sigma g(\pi x)=g(x)$ is an undecidable problem [253] (as notation, $\operatorname{dom}(f)$ is the domain of the function $f(x)$ ). Hence, we should try to find subgroups of $\bar{G}_{P}$ which can be computed automatically. Suppose there is an oracle that takes two functions as input and give an answer "yes" or "not". If the answer of the oracle is yes, the corresponding functions are equal, but the converse may not hold. This oracle is based on a representation of the functions using DAGs, thus two functions $a$ and $b$ are recognized to be equal only if their corresponding DAGs $T_{a}$ and $T_{b}$ are equal. However, only a subset of all the functions that are actually equal are recognized to be equal by the oracle. More precisely, the oracle can correctly establish for the equivalence of two functions only if they are strings of a formal language $\mathscr{L}$ on an alphabet consisting on the operators $\{+,-, \times, \div, \uparrow, \log , \exp ,()$,$\} (where a \uparrow b=a^{b}$ ), the variable symbols of the problem, and the constant symbols in $\mathbb{R}$. For example, the functions $x_{1}+x_{2}$ and $x_{2}+x_{1}$ produce an answer yes by the oracle, unlike the functions $\sin (x)$ and $\sqrt{1-\cos ^{2}(x)}$. For more details, see [63].

For $a, b \in \mathscr{L}$ we define $a \cong b$ if and only if $T_{a}=T_{b}$ : this can be established in linear time in $|a|,|b|$ by simply recursing on the respective DAGs. It is easy to show that if $a \cong b$ then $\operatorname{dom}(a)=\operatorname{dom}(b) \wedge \forall x \in \operatorname{dom}(a) a(x)=b(x)$ (thus the functions represented by the strings $a$ and $b$ are equal), but the converse may not hold. For a MP $P^{\prime}$ defined as:

$$
\begin{array}{cl}
\min & f^{\prime}(x) \\
\text { s.t. } & g^{\prime}(x) \leq 0 \\
& x \in X^{\prime},
\end{array}
$$

we write $P \cong P^{\prime}$ if: (a) $P, P^{\prime}$ have the same number of variables and constraints; (b) $X=X^{\prime}$; (c) $f \cong f^{\prime}$ and $\forall i \leq m\left(g_{i} \cong g_{i}^{\prime}\right)$. We are finally in a position to define the formulation group $G_{P}=\left\{\pi \in S_{n} \mid \exists \sigma \in S_{m}(\sigma P \pi \cong P)\right\}$ of $P$. It is easy to show that $G_{P} \leq \bar{G}_{P} \leq G_{P}^{*}[155]$. For MILPs, $G_{P}=\bar{G}_{P}[158]$. 
Example 3.2.2. Consider the following MILP problem:

$$
\begin{array}{cl}
\min & x_{1}+x_{2}+x_{3} \\
\text { s.t. } & x_{1}+x_{2} \geq 1 \\
& x_{1}+x_{3} \geq 1 \\
& x_{2}+x_{3} \geq 1 \\
& \forall j \in\{1,2,3\} \quad x_{j} \in\{0,1\} .
\end{array}
$$

The problem can be casted in the form

$$
\begin{array}{cl}
\min & c^{T} x \\
\text { s.t. } & A x \geq b \\
& x \in\{0,1\}^{3},
\end{array}
$$

where

$$
c^{T}=[111], \quad x=\left[\begin{array}{l}
x_{1} \\
x_{2} \\
x_{3}
\end{array}\right], \quad b=\left[\begin{array}{l}
1 \\
1 \\
1
\end{array}\right], \quad A=\left[\begin{array}{lll}
1 & 1 & 0 \\
1 & 0 & 1 \\
0 & 1 & 1
\end{array}\right]
$$

Consider the column permutation $\pi=(2,3)$, which swap variable $x_{2}$ with variable $x_{3}$. There exists a row permutation $\sigma=(1,2)$, which swaps the first and second constraints, such that $\sigma P \pi \cong P$. Actually $\sigma P \pi=P$ since the matrix constraints remains the same after swapping columns 2 and 3 (due to the permutation $\pi$ ), and the rows 1 and 2 (due to the permutation $\sigma$ ). The objective function does not change after applying the permutation $\pi$ on the variables, since all the coefficients are equal to 1. Thus, the permutation $\pi \in G_{P}$ (in this case, as the problem is a MILP, then it also holds that $\pi \in \bar{G}_{P}$ ).

\subsubsection{Symmetric structure of circle packing}

As described in the previous section (and in $[63,158]$ ) symmetries of MINLPs can be automatically detected by encoding the MINLP instance as a DAG and then finding the graph automorphisms group of this DAG. The group generators can then be "projected" on the set of variable indices, thus obtaining a set of generators for the group $G_{P}$ of variable permutations which keep the formulation of the MINLP $P$ invariant.

The results presented on Table 3.2 were obtained using a software system deployed on the PECS: (the experiments were conducted on many more instances). This allowed us to conjecture that the formulation group of the PECS formulation is $C_{2} \times S_{n}$. Intuitively, this is reasonable: $C_{2}$ corresponds to permuting the symbols $x$ with the symbols $y$ (that is, swapping $x$ and $y$ axes), and $S_{n}$ corresponds to 


\begin{tabular}{cl}
\hline$n$ & $G_{\mathrm{PECS}}$ \\
\hline 2 & $C_{2} \times S_{2}$ \\
3 & $C_{2} \times S_{3}$ \\
4 & $C_{2} \times S_{4}$ \\
5 & $C_{2} \times S_{5}$ \\
\hline
\end{tabular}

Table 3.2: Formulation group of PECS for some instances.

permuting the variable indices (that is, swapping some circles). The hardest part of proving the conjecture, of course, is showing that there are no other formulation symmetries for a generic $n$.

The proof structure is similar to the proof given in [159] for the Kissing Number Problem [143].

Theorem 3.2.3. The formulation group of the PECS problem is isomorphic to $C_{2} \times S_{n}$.

Proof. Let $G_{\mathrm{PECS}}$ be the formulation group of PECS. For all $i<j \leq n$ call the constraints $\left(x_{i}-x_{j}\right)^{2}+\left(y_{i}-y_{j}\right)^{2} \geq 4 r^{2}$ the distance constraints (3.2). Let $(x, y, r) \in$ $\mathcal{G}$ (PECS); the following claims are easy to establish.

1. The permutation $\tau=\prod_{i \leq n}\left(x_{i}, y_{i}\right)$ is in $G_{\mathrm{PECS}} ;\left(\langle\tau\rangle \cong C_{2}\right)$.

2. For any $i<n$, the permutation $\sigma_{i}=\left(x_{i}, x_{i+1}\right)\left(y_{i}, y_{i+1}\right)$ is in $G_{\text {PECS }}$; notice that $\left\langle\sigma_{i} \mid i<n\right\rangle \cong S_{n}$.

3. Any permutation moving $r$ to one of the variables $\notin G_{\text {PECS }}$.

4. If $\pi \in G_{\mathrm{PECS}}$ such that $\pi\left(x_{i}\right)=y_{i}$ for some $i \leq n$ then $\pi\left(x_{i}\right)=y_{i}$ for all $i \leq n$, as otherwise the term $x_{i} x_{j}+y_{i} y_{j}$ (appearing in the distance constraints) would be mapped to a term not appearing in the problem.

5. For any $i<n$, if $\pi \in G_{\text {PECS }}$ such that $\pi\left(z_{i}\right)=z_{i+1}$ for some $z \in\{x, y\}$, then $\pi\left(z_{i}\right)=z_{i+1}, \forall z \in\{x, y\}$; if not the term $x_{i} x_{i+1}+y_{i} y_{i+1}$ (appearing in some of the distance constraints) would be mapped to a term not appearing in the problem.

Let $K=\langle\tau\rangle$ and $H_{n}=\left\langle\sigma_{i} \mid i \leq n-1\right\rangle$. Claims (1)-(2) imply that $K, H_{n} \leq$ $G_{\text {PECS }}$. It is tedious but not too hard to check that $K H_{n}=H_{n} K$; it follows that $K H_{n} \leq G_{\text {PECS }}$ and hence $K, H_{n}$ are normal subgroups of $K H_{n}$. Since $K \cap H_{n}=\{e\}$, we have $K H_{n} \cong K \times H_{n} \cong C_{2} \times S_{n} \leq G_{\text {PECS }}$.

Now suppose $\pi \in G_{\mathrm{PECS}}$ with $\pi \neq e$. By Claim (3), $\pi$ cannot move $r$ so it must map $x_{i}$ to $y_{j}$ for some $i<j \leq n$; the action $i \rightarrow j$ on the circles indices can be decomposed into a product of transpositions $i \rightarrow i+1, \ldots, j-1 \rightarrow j$. Thus, by Claim (5) (resp. 4), $\pi$ involves a certain product $\gamma$ of $\tau$ and $\sigma_{i}$ 's; furthermore, 
since by definition $\gamma$ maps $x_{i}$ to $y_{j}$, any permutation in $G_{\text {PECS }}$ (including $\pi$ ) can be obtained as a product of these elements $\gamma$; hence $\pi$ is an element of $K H_{n}$, which shows $G_{\mathrm{PECS}} \leq K H_{n}$, implying $G_{\mathrm{PECS}} \cong C_{2} \times S_{n}$.

\subsection{Order symmetry breaking constraints}

Once $G_{P}$ is known, we aim to find a reformulation $Q$ of $P$ which ensures that at least one symmetric optimum of $P$ is in $\mathcal{G}(Q)$. Adjoining SBCs to $P$ yields a narrowing $Q$ of $P$ [158]. The formal definition of SBC is the following.

Definition 3.3.1. Symmetry Breaking Constraints (SBCs) A set of constraints $h(x) \leq 0$ are SBCs with respect to $\pi \in G_{P}$ if there is $y \in \mathcal{G}(P)$ such that $h(\pi y) \leq 0$.

Since Theorem 3.2.3 states that the formulation group of the PECS problem $G_{P E C S}$ is isomorphic to $C_{2} \times S_{n}$, we propose in the following some SBCs to break these symmetries.

\subsubsection{Weak constraints}

The first set of SBCs are obtained from the following consideration: let $\Omega$ be the set of nontrivial orbits of the action of $G_{P}$ on the set of variable symbols of a problem, and let $\omega \in \Omega$. Then $\forall j \in \omega x_{\min \omega} \leq x_{j}$ are SBCs with respect to $G_{P}$ [158].

Applying this to the PECS problem, we can define the following weak constraints (the name comes from the fact that they provide the smallest improvement among the SBCs proposed in this chapter):

$$
\forall 2 \leq j \leq n \quad x_{1} \leq x_{j}
$$

These SBCs are based on the fact that we can always choose a an arbitrary index (for example 1) such that the circle corresponding to that index is leftmost. One might alternatively choose to employ $\forall 2 \leq j \leq n y_{1} \leq y_{j}$. These SBCs were discussed in $[63]$.

\subsubsection{Strong constraints}

By Theorem 3.2.3, $G_{P E C S}=\left\langle\tau, \sigma_{i} \mid i \leq n-1\right\rangle$. It is easy to show that there is just one orbit in the natural action of $G_{P E C S}$ on the set $A=\{1, \ldots, n\} \times\{1,2\}$, and that the action of $G_{P E C S}$ on $A$ is not symmetric (otherwise $G_{P E C S}$ would be isomorphic to $S_{2 n}$, contradicting Theorem 3.2.3).

Proposition 3.3.2.

$$
\forall i<n \quad x_{i} \leq x_{i+1}
$$

are $S B C s$ with respect to any $\pi \in G_{P E C S}$. 
Proof. Let $\left(x^{*}, y^{*}, r^{*}\right) \in \mathcal{G}(P E C S)$; since the $\sigma_{i}$ generate the symmetric group acting on the $n$ circles, there exists a permutation $\pi \in G_{P E C S}$ such that $\left(x^{*}{ }_{\pi(i)} \mid i \leq n\right)$ are ordered as in (3.32).

We call (3.32) strong constraints, since they are more effective than weak constraints for removing symmetries of PECS, as showed by experiments carried out in [63]. These SBCs are based on the fact that the circles can be ordered on the horizontal axis. Again, one can alternatively employ $\forall i<n \quad y_{i} \leq y_{i+1}$.

It is interesting to see experimentally the effect of these SBCs on the solution process of the local solver employed by the sBB algorithm. As mentioned in Section 3.2 , we observe that good feasible solutions were found earlier in the search with SBCs rather than without.

All our experiments in this chapter are conducted using the Couenne sBB solver (trunk version dated November 2010) with the default configuration, which employs the IPOpT [244] subsolver as the local NLP solver used to find incumbents in Step 4 of the sBB algorithm given in Section 1.2.2.5. IPOPT actually solves the following PECS reformulation:

$$
\begin{aligned}
-\min & -r \\
\text { s.t. } & \forall i<j \leq n \quad\left(x_{i}-x_{j}\right)^{2}+\left(y_{i}-y_{j}\right)^{2}-4 r^{2}-s_{i, j}=0 \\
& \forall i \leq n \quad x_{i}-r-L_{i}^{x}=0 \\
& \forall i \leq n \quad y_{i}-r-L_{i}^{y}=0 \\
& \forall i \leq n \quad x_{i}+r-1+U_{i}^{x}=0 \\
& \forall i \leq n \quad y_{i}+r-1+U_{i}^{y}=0 \\
& \forall i<j \leq n \quad L_{i}^{x} \in \mathbb{R}_{0}^{+} \\
& \forall i<j \leq n \quad L_{i}^{y} \in \mathbb{R}_{0}^{+} \\
& \forall i<j \leq n \quad U_{i}^{x} \in \mathbb{R}_{0}^{+} \\
& \forall i<j \leq n \quad U_{i}^{y} \in \mathbb{R}_{0}^{+} \\
& \forall i<j \leq n \quad s_{i, j} \in \mathbb{R}_{0}^{+} \\
& \forall i \leq n \quad x_{i} \in \mathbb{R}_{0}^{+} \\
& \forall i \leq n \quad y_{i} \in \mathbb{R}_{0}^{+} \\
& r \in \mathbb{R}_{0}^{+},
\end{aligned}
$$

obtained by introducing slack variables for each inequality. The natural starting point for solving (3.33)-(3.46) in Step 4 is the solution of the relaxation in Step 2, which is $\forall i \leq n x_{i}=y_{i}=r=\frac{1}{2}$ at the root node by Proposition 3.2.1. Since this is infeasible with respect to (3.34), IPOPT starts with a feasibility restoration phase, converging to the starting point $\forall i \leq n x_{i}=y_{i}=\frac{1}{2}, r=0$. It is long and tedious, but easy, to check that Linear Independence Constraints Qualification 
(LICQ) conditions hold at this starting point, and which is therefore a KKT point. Thus, IPOpt simply confirms it as a local optimum, and this is consistent with the results in Table 3.3 (first column).

\begin{tabular}{ccccc}
\hline & \multicolumn{2}{c}{ no SBCs } & \multicolumn{2}{c}{ strong } \\
$n$ & $r$ & CPU & $r$ & CPU \\
\hline 4 & $4.5 \mathrm{e}-5$ & 1.9 & 0.25 & 0.07 \\
5 & $4.5 \mathrm{e}-5$ & 2.1 & 0.196 & 0.02 \\
6 & $5 \mathrm{e}-5$ & 0.05 & 0.187 & 0.04 \\
7 & $5 \mathrm{e}-5$ & 0.06 & 0.174 & 0.04 \\
8 & $5 \mathrm{e}-5$ & 0.05 & 0.169 & 0.06 \\
9 & $5 \mathrm{e}-5$ & 0.06 & 0.166 & 0.04 \\
10 & $5 \mathrm{e}-5$ & 0.06 & 0.148 & 0.06 \\
20 & $4.95 \mathrm{e}-5$ & 0.24 & 0.109 & 0.27 \\
50 & $4.89 \mathrm{e}-5$ & 48.91 & 0.068 & 4.82 \\
\hline
\end{tabular}

Table 3.3: IPOpT with starting point $\forall i \leq n x_{i}=y_{i}=0.5, r=0$ with and without strong SBCs.

If, on the other hand, we adjoin SBCs to the formulation, positive ascent directions are found using IPOPT's Second Order Corrections [244], as shown by the locally optimal $r$ values in the third column of Table 3.3. This is consistent with the intuitive explanation given in Section 3.2. Another interesting phenomenon occurs: the CPU time taken by IPOpT is reduced for the PECS formulation with SBCs (Table 3.3, second and fourth column). This is due to the fact that interior point methods require primal variables to have strictly positive values at each iteration [244], and $r=0$ obviously fails to satisfy this requirement. A different local NLP solver, snopt, which is based on a SQP method, converges a local optimum in roughly the same CPU time both with and without SBCs, but fails to find ascent directions for $r$, because it is a first-order method and does not exploit Second Order Corrections.

Although the above discussion only holds at the root node, further experiments with random variable bounds have shown that SBCs yield better values for $r$ at lower nodes too (although the marked difference in CPU time disappears).

\subsubsection{Mixed constraints}

In order to improve the strong SBCs (i.e., to make more symmetric optima infeasible), we propose the mixed SBCs, which contain constraint on the $x$ variables as well as constraint on the $y$ variables. To do that, we remove some of the strong SBCs in $x$ and replace them with compatible SBCs in $y$. Given any $L \in\left\{1, \ldots,\left\lfloor\frac{n}{2}\right\rfloor\right\}$, consider the strong SBCs. For each $i \in\left\{1,2, \ldots,\left\lceil\frac{n}{L}\right\rceil-1\right\}$ we replace the constraints $x_{i L} \leq x_{i L+1}$ with $y_{1+(i-1) L} \leq y_{1+i L}$.

In order to show that the mixed constraints are SBCs, we prove that the PECS 
formulation with the mixed constraints adjoined is a narrowing of the PECS formulation. We define the following index sets:

- $\mathcal{N}=\{1, \ldots, n\}$

- $\mathcal{N}^{\prime}=\{1, \ldots, n-1\}$

- $\mathcal{N}^{\prime \prime}=\{1, L+1,2 L+1, \ldots,(\lceil n / L\rceil-2) L+1\}$,

the following sets of constraints (intended as list of symbolic expressions representing the constraints, rather than sets of real vectors feasible with the constraints):

- $\mathscr{S}=\left\{x_{i} \leq x_{i+1} \mid i \in \mathcal{N}^{\prime}\right\}$

- $\forall i \in \mathcal{N}^{\prime \prime} \quad \mathscr{A}_{i}=\left\{x_{h} \leq x_{h+1} \mid h \in \mathcal{N}^{\prime} \backslash\{i+L-1\}\right\}$

- $\forall i \in \mathcal{N}^{\prime \prime} \quad \mathscr{C}_{i}=\left\{y_{i} \leq y_{i+L}\right\}$

and the following formulations:

- PECS $^{\prime} \equiv$ PECS $\cup \mathscr{S}$ (i.e., the PECS formulation with strong constraints)

- $\forall i \in \mathcal{N}^{\prime \prime} \quad \operatorname{PECS}_{i} \equiv \operatorname{PECS} \cup \mathscr{A}_{i} \cup \mathscr{C}_{i}$,

- $\operatorname{PECS}^{\prime \prime} \equiv \operatorname{PECS} \cup \bigcup_{i \in \mathcal{N}^{\prime \prime}}\left(\mathscr{A}_{i} \cup \mathscr{C}_{i}\right)$.

Proposition 3.3.3. For all $i \in \mathcal{N}^{\prime \prime}, P E C S_{i}$ is a narrowing of PECS.

Proof. Let $i \in \mathcal{N}^{\prime \prime}$ and $\left(x^{*}, y^{*}, r^{*}\right) \in \mathcal{G}$ (PECS). For a permutation $\pi \in S_{n}$ we assume $\pi\left(x^{*}, y^{*}, r^{*}\right)=\left(\pi x^{*}, \pi y^{*}, \pi r^{*}\right)$ where $\pi$ acts on a vector in $\mathbb{R}^{n}$ by permuting the indices of its components; notice that since $\pi$ is simply a reindexing of the circles, $\pi\left(x^{*}, y^{*}, r^{*}\right) \in \mathcal{G}(\mathrm{PECS})$. Furthermore, since PECS ${ }^{\prime}$ is known to be a narrowing of PECS, we can assume WLOG that $\left(x^{*}, y^{*}, r^{*}\right)$ satisfies $\mathscr{S}$. If $y^{*}{ }_{i} \leq y^{*}{ }_{i+L}$ the result holds, otherwise assume $y^{*}{ }_{i}>y^{*}{ }_{i+L}$. Consider the permutation $\sigma_{i}=\prod_{\ell=0}^{L-1}(i+$ $\ell, i+L+\ell)$ in $S_{n} ; \sigma_{i}\left(x^{*}, y^{*}, r^{*}\right)$ has the following properties: (a) by the action of the 2-cycle $(i, i+L)$ (appearing in $\sigma_{i}$ when $\ell=0$ ) we have $y^{*}{ }_{i}<y^{*}{ }_{i+L}$; (b) $\forall \ell \in\{0, \ldots, L-2\}$ we have $\sigma_{i} x^{*}{ }_{i+\ell}=x^{*}{ }_{i+L+\ell} \leq x^{*}{ }_{i+L+\ell+1}=\sigma_{i} x^{*}{ }_{i+\ell+1}$ and $\sigma_{i} x^{*}{ }_{i+L+\ell}=x^{*}{ }_{i+\ell} \leq x^{*}{ }_{i+\ell+1}=\sigma_{i} x^{*}{ }_{i+L+\ell+1}$; (c) $\forall h \in \mathcal{N}^{\prime}$ such that $h \notin H_{i}=$ $\{i, \ldots, i+2 L-1\}$ we have $\sigma_{i} x^{*}{ }_{h}=x^{*}{ }_{h} \leq x^{*}{ }_{h+1}=\sigma_{i} x^{*}{ }_{h+1}$ because $\sigma_{i}$ fixes all $h \notin H_{i}$. Thus $\sigma_{i}\left(x^{*}, y^{*}, r^{*}\right) \in \mathcal{G}(\mathrm{PECS})$ and satisfies the constraints of $\operatorname{PECS}_{i}$.

Lemma 3.3.4. Let $t=\lceil n / L\rceil-1$ and $\Sigma=\left\{\sigma_{i} \mid i \in \mathcal{N}^{\prime \prime}\right\}$. Then $\langle\Sigma\rangle \cong S_{t}$.

Proof. Notice $\mathcal{N}^{\prime \prime}=\{(j-1) L+1 \mid 1 \leq j \leq t\}$, and define a map $\varphi((j-1) / L+1)=$ $j$, under which $\varphi(\Sigma)=\{(1,2),(2,3), \ldots,(t-1, t)\}$. This map induces a group homomorphism $\bar{\varphi}:\langle\Sigma\rangle \rightarrow S_{t}$ given by $\bar{\varphi}\left(\sigma_{i}\right)=(\varphi(i), \varphi(i)+1)$, which can be verified to be injective and surjective. 
Similarly, for all $h<k \in \mathcal{N}^{\prime \prime}$ we have $\left\langle\Sigma^{h k}\right\rangle=\left\langle\left\{\sigma_{i} \mid h \leq i<k\right\}\right\rangle \cong \operatorname{Sym}\left(I^{h k}\right)$, the symmetric group on the set $I^{h k}=\{\varphi(h), \ldots, \varphi(k)\}$. Thus, for all $h, k \in \mathcal{N}^{\prime \prime}$, the permutation $\tau_{h k}=\prod_{\ell=0}^{L-1}(h+\ell, k+\ell)$ can be obtained as a certain product of the $\sigma_{i}$ 's for $i \in \varphi^{-1}\left(I^{h k}\right)$. More precisely, we have $\tau_{h k}=(\varphi(k)-1, \varphi(k))(\varphi(k)-$ $2, \varphi(k)-1) \cdots(\varphi(h), \varphi(h)+1)(\varphi(h)+1, \varphi(h)+2) \cdots(\varphi(k)-1, \varphi(k))$.

Theorem 3.3.5. PECS" is a narrowing of PECS.

Proof. Let $\left(x^{*}, y^{*}, r^{*}\right) \in \mathcal{G}$ (PECS), and consider the set $\mathscr{V}$ of all constraints $\mathscr{C}_{i} \equiv$ $\left\{y_{i} \leq y_{i+L}\right\}$ violated by $\left(x^{*}, y^{*}, r^{*}\right)$. Let $\psi$ be the (invertible) map given by $\psi\left(\mathscr{C}_{i}\right)=$ $(\varphi(i), \varphi(i)+1)$; then $\psi(\mathscr{V})$ is a set of transpositions that can be partitioned into maximal non-disjoint subsets $S^{h k}=\{(\varphi(h), \varphi(h)+1), \ldots,(\varphi(k)-1, \varphi(k))\}$; let $\mathscr{T}$ be the set of pairs $(h, k)$ for which $S^{h k}$ is in the partition of $\psi(\mathscr{V})$. It is easy to verify that if $\pi_{h k}=\prod_{\substack{\ell \in I^{h k} \\ h+\ell L<k-\ell L}} \tau_{h+\ell L, k-\ell L}$ then $\pi_{h k} y^{*}$ satisfies the constraints in $\psi^{-1}\left(S^{h k}\right)$. Furthermore, by maximality of the $S^{h k}$, the permutations $\pi_{h k}$ are disjoint. Now, if $\pi=\prod_{(h, k) \in \mathscr{T}} \pi_{h k}, \pi\left(x^{*}, y^{*}, r^{*}\right)$ is such that $\pi y^{*}$ satisfies all constraints in $\mathscr{V}$ and $\pi x^{*}$ satisfies all constraints in $\bigcup_{i \in \mathcal{N}^{\prime \prime}} \mathscr{A}_{i}$ by Proposition 3.3.3. Thus $\pi\left(x^{*}, y^{*}, r^{*}\right) \in$ $\mathcal{G}\left(\mathrm{PECS}^{\prime \prime}\right)$.

\subsubsection{Numerical results}

It has been shown in [59] that mixed constraints are more effective to remove symmetries than strong constraints, so we consider now the PECS model with mixed SBCs.

First, from previous section it appears that mixed SBCs rely on an arbitrary choice for the integer $L$. Figure 3.4 shows the number of sBB tree nodes in function of $L$ for the instances from $n=4$ to $n=9$. These experiments indicate that $L=2$ is the best choice (Note that the choice $L=1$ is not considered since it corresponds to removing all the strong constraints on the $x$ variables and to adjoin the corresponding constraints on the $y$ variables).

In order to test the mixed constraints, we first provide empirical evidence that the proposed SBCs tighten the upper bound in Step 2 of Section 1.2.2.5 by solving a set of small PECS instances to global optimality using the CoUENNE solver on a $2.4 \mathrm{GHz}$ Intel Xeon CPU with 24 GB RAM running Linux. Table 3.4 reports the instance $(n)$, the globally maximum possible radius $r^{*}$ allowing a packing of $n$ circles in the unit square, the number of sBB nodes, and the seconds of user CPU time taken by CouENNE running to termination on the original formulation and on the narrowing.

A second set of tests concerns the performance of Couenne on the mixed SBC based narrowing with early termination based on two hours of user CPU time. In Table 3.5 we report the number of circles, the best known solution $\bar{r}$ (taken from http://www . packomania.com; a proof of optimality is only given for instances where 

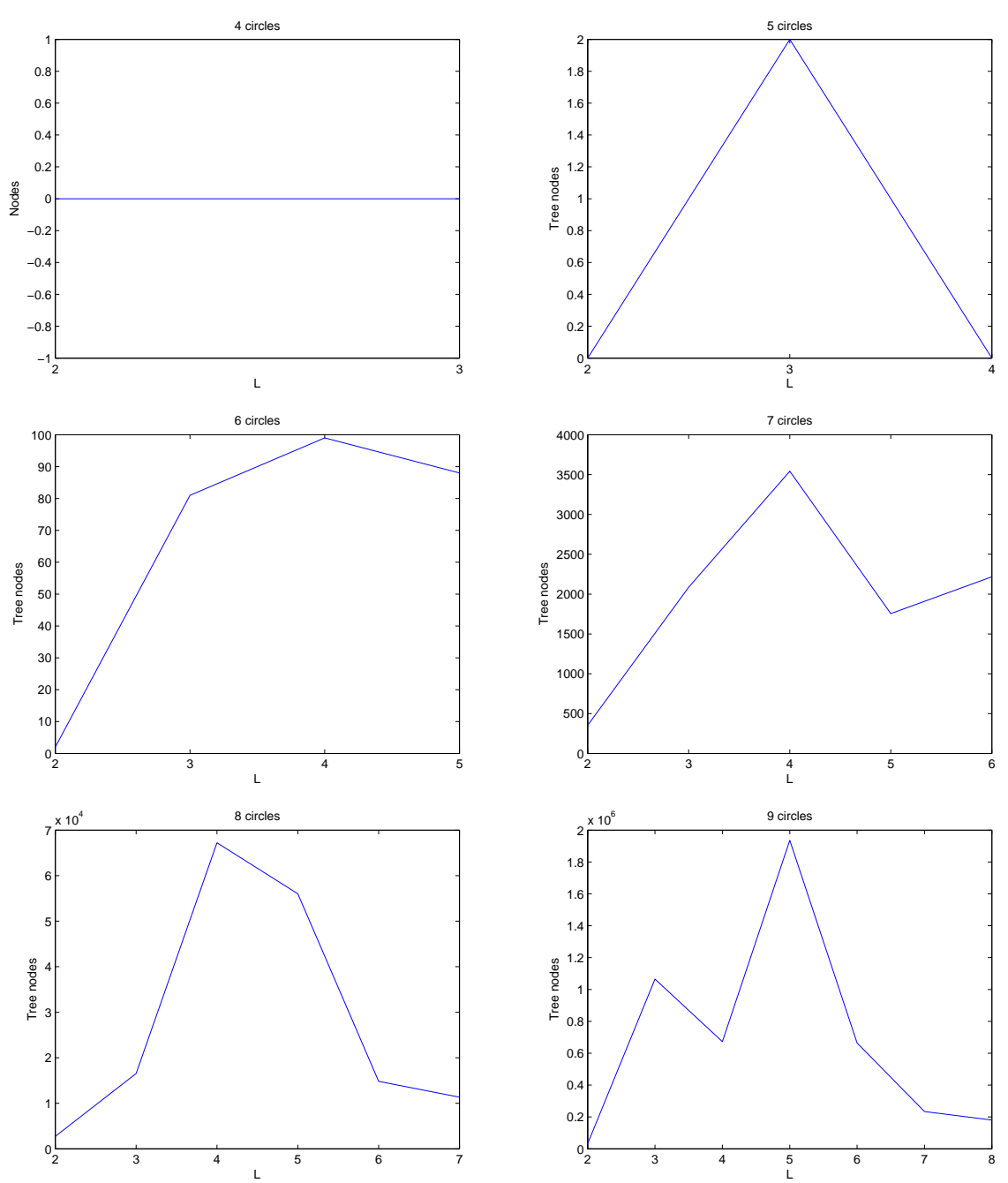

Figure 3.4: sBB tree nodes in function of $L$.

\begin{tabular}{llrrrr}
\hline & & \multicolumn{2}{c}{ Original formulation } & \multicolumn{2}{c}{ Mixed SBC Narrowing } \\
$n$ & $r^{*}$ & sBB nodes & CPU time & sBB nodes & CPU time \\
\hline 2 & 0.292893 & 2 & 0.04 & $\mathbf{0}$ & $\mathbf{0 . 0 2}$ \\
3 & 0.254333 & 2 & 0.15 & $\mathbf{0}$ & $\mathbf{0 . 0 8}$ \\
4 & 0.25 & 282 & 1.85 & $\mathbf{0}$ & $\mathbf{0 . 0 8}$ \\
5 & 0.207113 & 68710 & 69.24 & $\mathbf{5 4 1}$ & $\mathbf{2 . 0 2}$ \\
6 & 0.187707 & 3087798 & 6176.05 & $\mathbf{4 2 8 5 0}$ & $\mathbf{9 0 . 8 4}$ \\
\hline
\end{tabular}

Table 3.4: sBB running to termination on small PECS instances.

$n \leq 30$ and $n=36$ ), the solution found at the root node $r_{r}$, the largest radius $\check{r}$ found by our method within the time limit, the tightest upper bound $\hat{r}$ (which gives an idea of the optimality gap), the time $t(\check{r})$ at which the solution $\check{r}$ was found, and the number of nodes explored within the time limit.

The mixed SBCs both tighten the problem relaxation and, rather unexpectedly, 


\begin{tabular}{lllllrr}
\hline$n$ & $\bar{r}$ & $r_{r}$ & $\check{r}$ & $\hat{r}$ & $t(\check{r})$ & sBB nodes \\
\hline 20 & 0.111382 & 0.111382 & 0.111382 & 0.322063 & 16.45 & 441828 \\
25 & 0.1 & 0.096852 & 0.1 & 0.250133 & 553.68 & 125632 \\
30 & 0.091671 & 0.091671 & 0.091671 & 0.316273 & 86.24 & 90230 \\
35 & 0.084290 & 0.082786 & 0.083766 & 0.351545 & 1495.31 & 46162 \\
40 & 0.079186 & 0.078913 & 0.078913 & 0.2501 & 19.68 & 17116 \\
45 & 0.074727 & 0.07444 & 0.07444 & 0.353325 & 357.90 & 12915 \\
50 & 0.071377 & 0.070539 & 0.070539 & 0.250121 & 5429.88 & 2 \\
\hline
\end{tabular}

Table 3.5: sBB running on large PECS instances.

ease the work of the local solver deployed at each node. An interesting fact is that the formulation with mixed SBCs leads to solutions very close to the best known already at the root node of the sBB tree, as shown in Table 3.5.

\subsection{Other constraints}

In this section we present other constraints which are useful to tighten the PECS formulation. We consider as starting model the PECS formulation (3.1)-(3.5) with the strong SBCs (3.32) adjoined. We use the strong constraints because they are necessary to derive some of the inequalities presented in the following.

\subsubsection{Fixing points symmetry breaking constraints}

In [173], the authors present the following theorem for PPS (a proof can be found in $[172,209])$ :

Theorem 3.4.1. There always exists an optimal solution of the PPS problem such that at each vertex $v$ of the unit square, incident to the sides $e_{1}$ and $e_{2}$, one and only one of the following statements holds:

- a point of the optimal solution is in the vertex $v$;

- two points of the optimal solution belong to the sides $e_{1}$ and $e_{2}$ and have distance equal to the optimal one.

Starting from this theorem we can prove the following:

Theorem 3.4.2. Consider the PPS problem with $n \geq 4$. There is always an optimal solution where at least two points are on the left side of the square, and at least two points are on the right side of the square.

Proof. Consider the left side of the square, and call $v_{1}$ the bottom-left vertex, while $v_{2}$ is the top-left one; by Theorem 3.4.1, we can have four different situations:

(a) we have a point $\left(p_{1}\right)$ in $v_{1}$ and one $\left(p_{2}\right)$ in $v_{2}$; 
(b) we have a point $\left(p_{1}\right)$ in $v_{1}$, and we have 2 other points: one on the left side of the square $\left(p_{2}\right)$, one on the top side $\left(p_{3}\right)$ whose distance is the optimal one $m^{*}$;

(c) we have a point $\left(p_{2}\right)$ in $v_{2}$, and we have 2 other points: one on the left side of the square $\left(p_{1}\right)$, one on the bottom side $\left(p_{4}\right)$ whose distance is the optimal one $m^{*}$;

(d) we have one point on the left side of the square $\left(p_{2}\right)$ and one on the top side $\left(p_{3}\right)$ whose distance is the optimal one $m^{*}$; furthermore, we have another point on the left side $\left(p_{1}\right)$ and one on the bottom side $\left(p_{4}\right)$ whose distance is the optimal one $m^{*}$.

In all these cases, that are presented in Figure 3.5, we have at least two points on the left side of the square.

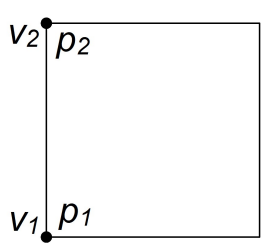

(a)

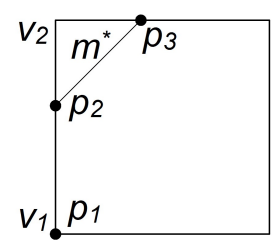

(b)

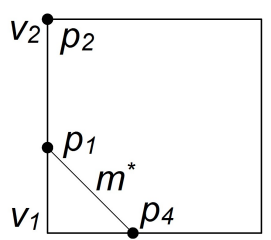

(c)

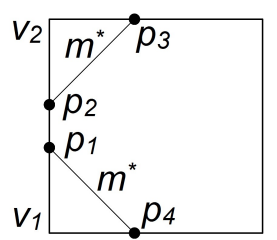

(d)

Figure 3.5: Possible configurations of points in the optimal solution of PPS according to Theorem 3.4.1.

All that remains to be shown is that in cases $3.5(\mathrm{~b}), 3.5(\mathrm{c}), 3.5(\mathrm{~d})$, the points $p_{1}$ and $p_{2}$ cannot coincide. For cases (b) and (c) if $p_{1}=p_{2}$ then $m^{*}>1$. This is not possible since the optimal distance when $n=4$ is equal to 1 , and for larger instances the distance decreases. Consider now the case (d). Suppose that $p_{1}=p_{2}$, that $v_{1}$ has coordinates $(0,0)$ and call $y_{a}$ the distance between $v_{1}$ and $p_{1}$ (hence the distance between $p_{1}$ and $v_{2}$ is equal to $\left.1-y_{a}\right)$. The distance $m^{*}$ is equal to the distance between $p_{1}$ and $p_{4}$, and the coordinate $x$ of $p_{4}$ is in $(0,1)$. Similarly, $m^{*}$ is equal to the distance between $p_{1}$ and $p_{3}$, and the coordinate $x$ of $p_{3}$ is in $(0,1)$. Hence the following inequalities hold:

$$
\begin{aligned}
& 1-y_{a}<m^{*}<\sqrt{1+\left(1-y_{a}\right)^{2}} \\
& y_{a}<m^{*}<\sqrt{y_{a}^{2}+1}
\end{aligned}
$$

where $y_{a} \in(0,1)$. Comparing these inequalities, it turns out that in order to have a valid value of $m^{*}$ the intervals $\left[1-y_{a}, \sqrt{1+\left(1-y_{a}\right)^{2}}\right]$ and $\left[y_{a}, \sqrt{y_{a}^{2}+1}\right]$ must have a nonempty intersection. To ease the following steps we can apply the square 
operator to the previous inequalities (since all the terms are positive), thus obtaining:

$$
\begin{aligned}
& \left(1-y_{a}\right)^{2}<m^{* 2}<1+\left(1-y_{a}\right)^{2} \\
& y_{a}{ }^{2}<m^{* 2}<y_{a}{ }^{2}+1
\end{aligned}
$$

In order to check that the intersection is nonempty, one should derive an order relationship between the left and right-hand sides of these inequalities. Since $y_{a} \in$ $(0,1)$, we have actually 3 cases to consider:

- $y_{a} \in\left(0, \frac{1}{2}\right)$. In this case the order relationship is the following: $y_{a}{ }^{2}<(1-$ $\left.y_{a}\right)^{2}<y_{a}^{2}+1<1+\left(1-y_{a}\right)^{2}$. Thus, $m^{* 2}$ must be between $\left(1-y_{a}\right)^{2}$ and $y_{a}^{2}+1$. In other words, we have that $1-y_{a}<m^{*}<\sqrt{y_{a}^{2}+1}, y_{a} \in\left(0, \frac{1}{2}\right)$;

- $y_{a} \in\left(\frac{1}{2}, 1\right)$. In this case the order relationship is the following: $\left(1-y_{a}\right)^{2}<$ $y_{a}{ }^{2}<1+\left(1-y_{a}\right)^{2}<y_{a}{ }^{2}+1$. Thus, $m^{* 2}$ must be between $y_{a}{ }^{2}$ and $1+\left(1-y_{a}\right)^{2}$. In other words, we have that $y_{a}<m^{*}<\sqrt{1+\left(1-y_{a}\right)^{2}}, y_{a} \in\left(\frac{1}{2}, 1\right)$;

- $y_{a}=\frac{1}{2}$. In this case we have $\left(1-y_{a}\right)^{2}=y_{a}{ }^{2}=\frac{1}{4}$ and $1+\left(1-y_{a}\right)^{2}=y_{a}{ }^{2}+1=\frac{5}{4}$. Hence, $\frac{1}{2}<m^{*}<\frac{\sqrt{5}}{2}$

In all these cases, the resulting inequality is the following:

$$
\frac{1}{2}<m^{*}<\frac{\sqrt{5}}{2}
$$

This means that we can have $p_{1}=p_{2}$ only if the optimal distance satisfies the inequality (3.47). We are considering the instances having $n \geq 4$. When $n=4$, the optimal distance is 1 , that is less than $\frac{\sqrt{5}}{2}$. The optimal distance when $n=9$ is equal to $\frac{1}{2}$, and for larger instances the optimal distance decreases. This means that the only possibilities for having $p_{1}=p_{2}$ are the instances where $4 \leq n \leq 8$. However, in these cases the optimal solutions are known, and there are always at least 2 points on a side of the square, as can be checked in [237] or in http://www.packomania.com. Hence, it is not possible that $p_{1}$ and $p_{2}$ coincide.

A similar idea can be used to prove the same for the right side of the square. Moreover, it is true even if we consider the other pair of opposite sides (that is top/bottom) in place of the left/right ones.

This result can be extended to the PECS problem in order to obtain some constraints, as proved by the following corollary.

Corollary 3.4.3. Consider the PECS problem with $n \geq 4$, where the strong SBCs (3.32) hold. The following constraints are valid:

$$
\begin{aligned}
& \forall i \in\{1,2\} \quad x_{i}=r \\
& \forall i \in\{n-1, n\} \quad x_{i}=1-r .
\end{aligned}
$$


Proof. Using the result of Theorem 3.4.2 and looking at Figure 3.1 it is obvious that for PECS problem there is always an optimal solution where at least two points are at distance $r$ from the left side of the square, and at least two points are at distance $r$ from the right side of the square. Thus, we can fix 2 points at distance $r$ to the left side of the square, and other 2 points at distance $r$ from the right side of the square. Since we want to respect also the strong SBCs (3.32), we can express that by means of the constraints (3.48) and (3.49).

\subsubsection{Bounds symmetry breaking constraints}

As remarked in [11], the following statements hold WLOG: ${ }^{1}$

- at least $n_{x}=\left\lceil\frac{n}{2}\right\rceil$ points are on the left half of the square ( $x$ bound constraints);

- among the previous $n_{x}$ points, at least $n_{y}=\left\lceil\frac{n_{x}}{2}\right\rceil$ are on the bottom half $(y$ bound constraints).

Unfortunately, this is not true if we have also the strong SBCs: for example, the optimal solution of the PECS problem when $n=8$ does not respect all these constraints together. In fact, as can be seen in Figure 3.6, if the solution respects both the strong SBCs and the $x$ bound constraints we cannot have the circles 1 and 2 in the bottom half of the square (that is $y_{1} \leq \frac{1}{2}$ and $y_{2} \leq \frac{1}{2}$, since $n_{y}=2$ ), so the $y$ bound constraints do not hold.

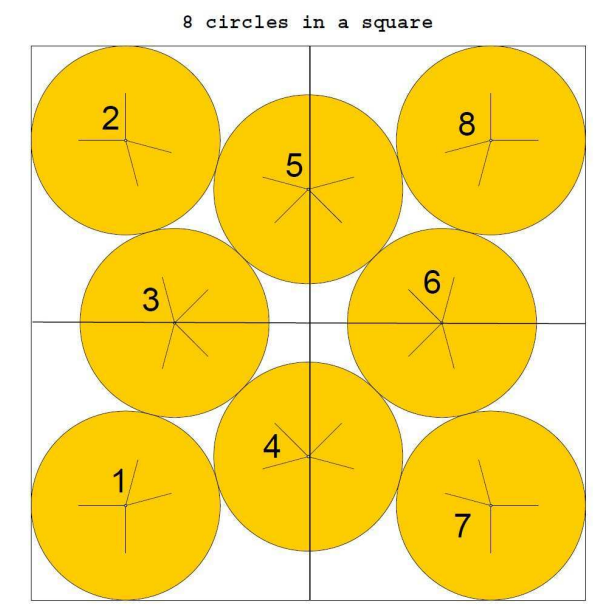

Figure 3.6: Optimal solution of PECS for $n=8$ (this figure is taken from http://www.packomania.com).

We can conclude that the $x$ bound constraints can be adjoined to the PECS model with strong SBCs, but not together with the $y$ bound constraints. Actually,

\footnotetext{
${ }^{1}$ The symmetric condition consisting of placing the first $n_{x}$ points on the right half of the square and then the first $n_{y}$ on the top half, considered in [11], is equivalent to the one presented here. This is because in that paper the strong constraints were considered with opposite sign, that is $x_{i} \geq x_{i+1}$ instead of $x_{i} \leq x_{i+1}$ as defined in (3.32).
} 
as claimed in [11], it is possible to have together the strong SBCs, the $x$ bound constraints, and the $y$ bound constraints if we drop the strong SBC $x_{n_{y}} \leq x_{n_{y}+1}$. However, we need to preserve all the strong SBCs to derive the "triangular inequality constraints" presented in Section 3.4.3.

Hence, we show how to formulate in another way the $y$ bound constraints, in order to add them to the model, and how to add the $x$ bound constraints using a single inequality.

The latter can be done this way: since the strong SBCs hold, it is sufficient to add the following inequality:

$$
x_{n_{x}} \leq \frac{1}{2}
$$

Thus, the inequalities $\forall i \leq n_{x} \quad x_{i} \leq \frac{1}{2}$ are automatically satisfied.

The former problem is basically the following: among the $n_{x}$ points that are on the left half of the square, at least $n_{y}$ are on the bottom half, but we cannot know which points are on the bottom half; nevertheless, we can obtain an inequality on the sum of the $y$ components of the first $n_{x}$ points.

More precisely, $n_{y}$ points have the coordinates $y$ which are smaller than or equal to $\frac{1}{2}$. For the others $n_{x}-n_{y}$ the $y$ coordinates are smaller than or equal to $1-r$. Hence, we can write the following inequality:

$$
\sum_{i=1}^{n_{x}} y_{i} \leq \frac{n_{y}}{2}+\left(n_{x}-n_{y}\right)(1-r)
$$

Using the same idea, we can obtain something similar for the sum of the $x$ components of all the points.

Basically, $n_{x}$ points have the coordinates $x$ that are smaller than or equal to $\frac{1}{2}$; among them, two have coordinates fixed to $r$, as shown by (3.48). For the others $n-n_{x}$ the $x$ coordinates are smaller than or equal to $1-r$. So, we can write this inequality:

$$
\sum_{i=1}^{n} x_{i} \leq \frac{1}{2}\left(n_{x}-2\right)+2 r+\left(n-n_{x}\right)(1-r) .
$$

It is interesting to notice that the constraint (3.52) might seem redundant if we have the constraints (3.3), (3.32), (3.48), and (3.50). Actually, some tests show that this inequality helps to obtain better upper bounds, above all with big instances of PECS. The reason for this behavior could be that Couenne uses this constraint to derive some cuts, which are automatically adjoined to the MP model.

\subsubsection{Triangular inequality constraints}

From the triangular inequality, we can write:

$$
\forall i<j \leq n \quad\left|x_{j}-x_{i}\right|+\left|y_{j}-y_{i}\right| \geq d_{i j} \geq 2 r
$$


where $d_{i j}$ represents the distance between the centers of the circles $i$ and $j$.

The strong SBCs imply that $\forall i<j \leq n x_{j}-x_{i} \geq 0$. Hence, we can remove the absolute value on the $x$ variables from (3.53) obtaining:

$$
\forall i<j \leq n \quad x_{j}-x_{i}+\left|y_{j}-y_{i}\right| \geq 2 r
$$

Our aim is to remove the absolute value from the $y$ variables, since it is a source of nonlinearity and makes the inequality difficult to solve. In order to get the final set of constraints, we should prove the following proposition:

Proposition 3.4.4. Given the constraints (3.2)-(3.5) of the PECS formulation and the strong SBCs, the following inequalities hold:

$$
\forall i<j \leq n \quad y_{j}+y_{i} \geq\left|y_{j}-y_{i}\right|+2 r .
$$

Proof. We can suppose WLOG that $y_{j} \geq y_{i}$ (if not the proof produces a similar result). Hence $\forall i<j \leq n y_{j}+y_{i} \geq y_{j}-y_{i}+2 r$. This is equivalent to $\forall i<j \leq$ $n y_{i} \geq r$, that is obviously true, since these inequalities are implied by (3.4).

At this point, we can remove the absolute value on the $y$ variables from (3.54) by replacing $\left|y_{j}-y_{i}\right|$ with $y_{j}+y_{i}-2 r$ :

$$
\forall i<j \leq n \quad x_{j}-x_{i}+y_{j}+y_{i}-2 r \geq x_{j}-x_{i}+\left|y_{j}-y_{i}\right| \geq 2 r .
$$

Finally we obtain the constraints:

$$
\forall i<j \leq n \quad x_{j}-x_{i}+y_{j}+y_{i} \geq 4 r
$$

\subsubsection{Numerical results}

In this section we compare two formulations of PECS for the instances where $4 \leq$ $n \leq 20$ : the original formulation (3.2)-(3.5) with the strong SBCs (PECS + strong), and the same formulation with all the new constraints proposed in Section 3.4, i.e., (3.48)-(3.52) and (3.55) (PECS + all). Our comparative results, shown in Table 3.6, have been obtained on a $2.4 \mathrm{GHz}$ Intel Xeon CPU with 24 GB RAM running Linux and the solver Couenne; the table displays the following statistics for the two formulations: objective function value $f^{*}$ of the incumbent, gap still open (we use the CPLEX definition [135]: $\left(\frac{100 \cdot\left|f^{*}-f_{U B}\right|}{\left|f *+10^{-10}\right|}\right) \%$, where $f_{U B}$ is the best upper bound found in the case of maximization problems), number of BB nodes closed, number of $\mathrm{BB}$ nodes still on the tree, and the CPU time (in seconds) taken, with a time limit of $2 \mathrm{~h}$. Moreover, we show also the optimal solutions $r^{*}$ for the instances, which can be found in [237] or in http://www.packomania.com.

The new constraints proposed in this section increase significantly the performance of COUEnNe with respect to the PECS formulation with strong SBCs, as 


\begin{tabular}{|c|c|c|c|c|c|c|c|c|c|}
\hline$n$ & $r^{*}$ & $f *$ & $\begin{array}{r}\text { PECS } \\
\text { gap }\end{array}$ & $\begin{array}{l}\text { strong } \\
\text { n. closed } \\
\text { n. on tree }\end{array}$ & CPU time & $f *$ & $\begin{array}{l}\text { PEC } \\
\text { gap }\end{array}$ & $\begin{array}{l}+ \text { all } \\
\text { n. closed } \\
\text { n. on tree }\end{array}$ & CPU time \\
\hline 4 & 0.25 & 0.25 & $0 \%$ & $\begin{array}{l}0 \\
0\end{array}$ & 0.12 & 0.25 & $0 \%$ & $\begin{array}{l}0 \\
0\end{array}$ & 0.13 \\
\hline 5 & 0.207107 & 0.207107 & $0 \%$ & $\begin{array}{r}2 \\
0 \\
\end{array}$ & 0.44 & 0.207107 & $0 \%$ & $\begin{array}{r}2 \\
0\end{array}$ & 0.19 \\
\hline 6 & 0.187681 & 0.187703 & $0 \%$ & $\begin{array}{c}8456 \\
0 \\
245102\end{array}$ & 17.90 & 0.187713 & $0 \%$ & $\begin{array}{c}110 \\
0 \\
564\end{array}$ & 7.25 \\
\hline 7 & 0.174458 & 0.174458 & $0 \%$ & $\begin{array}{c}0 \\
1853359\end{array}$ & 728.69 & 0.174458 & $0 \%$ & $\begin{array}{c}0 \\
7822\end{array}$ & 17.11 \\
\hline 8 & 0.170541 & 0.170541 & $17.71 \%$ & $\begin{array}{c}117869 \\
1365445\end{array}$ & 7200 & 0.170541 & $0 \%$ & $\begin{array}{c}0 \\
66070\end{array}$ & 65.78 \\
\hline 9 & 0.166667 & 0.166667 & $30.55 \%$ & $\begin{array}{c}279773 \\
1230472\end{array}$ & 7200 & 0.166667 & $0 \%$ & $\begin{array}{c}0 \\
611560\end{array}$ & 525.75 \\
\hline 10 & 0.148204 & 0.148201 & $65.10 \%$ & $\begin{array}{c}334114 \\
1068775\end{array}$ & 7200 & 0.148204 & $32.22 \%$ & $\begin{array}{l}201488 \\
498050\end{array}$ & 7200 \\
\hline 11 & 0.142399 & 0.142399 & $75.62 \%$ & 290037 & 7200 & 0.142339 & $39.61 \%$ & 179367 & 7200 \\
\hline 12 & 0.139959 & 0.139959 & $78.64 \%$ & 273315 & 7200 & 0.139959 & $59 \%$ & 136656 & 7200 \\
\hline 13 & 0.133994 & 0.133993 & $110.67 \%$ & $\begin{array}{l}816573 \\
232735 \\
615348\end{array}$ & 7200 & 0.133993 & $53.57 \%$ & $\begin{array}{l}337112 \\
133403 \\
250406\end{array}$ & 7200 \\
\hline 14 & 0.129332 & 0.129332 & $119.10 \%$ & $\begin{array}{l}182939 \\
853025\end{array}$ & 7200 & 0.129332 & $74.04 \%$ & $\begin{array}{c}97740 \\
204853\end{array}$ & 7200 \\
\hline 15 & 0.127167 & 0.126478 & $124.75 \%$ & $\begin{array}{l}245904 \\
382247\end{array}$ & 7200 & 0.127167 & $77.19 \%$ & $\begin{array}{c}81901 \\
173767\end{array}$ & 7200 \\
\hline 16 & 0.125 & 0.125 & $100.38 \%$ & $\begin{array}{l}121598 \\
275094\end{array}$ & 7200 & 0.125 & $77.40 \%$ & $\begin{array}{c}70580 \\
148004\end{array}$ & 7200 \\
\hline 17 & 0.117197 & 0.116293 & $115.19 \%$ & $\begin{array}{c}98707 \\
433224\end{array}$ & 7200 & 0.117111 & $91.16 \%$ & $\begin{array}{c}61668 \\
129641\end{array}$ & 7200 \\
\hline 18 & 0.115521 & 0.113218 & $175.46 \%$ & $\begin{array}{l}140861 \\
454058\end{array}$ & 7200 & 0.115521 & $101.74 \%$ & $\begin{array}{c}53367 \\
111486\end{array}$ & 7200 \\
\hline 19 & 0.112265 & 0.11174 & $179.20 \%$ & $\begin{array}{l}158505 \\
342260\end{array}$ & 7200 & 0.111911 & $104.83 \%$ & $\begin{array}{c}44392 \\
90274\end{array}$ & 7200 \\
\hline 20 & 0.111382 & 0.111382 & $210.63 \%$ & 116599 & 7200 & 0.111382 & $108.65 \%$ & 35542 & 7200 \\
\hline
\end{tabular}

Table 3.6: Results obtained by running Couenne on some PECS instances.

shown in Table 3.6. As a matter of fact, the time to obtain the optimal solution is lower, and when the time limit is reached for both formulations, the gap is smaller. This means that the formulation "PECS + all" leads to a lower value of the upper bound for $r$ (since the value of the best solution found by "PECS + all" is always greater than or equal to that of the "PECS + strong" formulation).

Looking at the number of nodes on the sBB tree, we can see that the trees associated to the "PECS + all" formulation are smaller than the trees obtained with "PECS + strong", as expected. Furthermore, in four cases the incumbent found with the "PECS + all" formulation is better than the one found with the "PECS + strong" formulation (in two cases, $n=15$ and $n=18$, the value is equal to the optimum). Hence, even if we test these formulations on a small number of instances, it is quite evident that "PECS + all" outperforms "PECS + strong".

Looking at the $n=6$ case in the table, we see that the incumbent values found are higher than the optima, but this is due to the numerical approximation of Couenne.

\subsection{A conjecture about the reduction of the search space}

In this section we present a conjecture about the tightening of some of the bound of the variables of PPS. The extension to the PECS problem is presented at the end. When we try to solve the PPS problem by means of sBB, usually the root node 
corresponds to a linear relaxation of the problem, whose optimal solution represents an upper bound for the original problem. For the PPS formulation (3.6)-(3.10) the relaxation is the following (as explained in $[173,209]$ ):

$$
\begin{aligned}
\max & \alpha \\
\text { s.t. } & \forall i<j \leq n \quad-l(i, j) \geq \alpha \\
& \forall i \leq n \quad x_{i} \in[0,1] \\
& \forall i \leq n \quad y_{i} \in[0,1] \\
& \alpha \in \mathbb{R}_{0}^{+},
\end{aligned}
$$

where $l(i, j)=-\left(L_{x_{i}}-U_{x_{j}}+U_{x_{i}}-L_{x_{j}}\right)\left(x_{i}-x_{j}\right)-\left(L_{y_{i}}-U_{y_{j}}+U_{y_{i}}-L_{y_{j}}\right)\left(y_{i}-\right.$ $\left.y_{j}\right)+\left(L_{x_{i}}-U_{x_{j}}\right)\left(U_{x_{i}}-L_{x_{j}}\right)+\left(L_{y_{i}}-U_{y_{j}}\right)\left(U_{y_{i}}-L_{y_{j}}\right)$ represents the convex envelope of the nonlinear part of constraint (3.2), while $L_{x_{i}}, L_{y_{i}}, U_{x_{i}}$, and $U_{y_{i}}$ represent the lower and upper bounds on the $x$ and $y$ variables (in this case, the lower bounds are equal to 0 and the upper bounds are equal to 1 for all the variables).

Proposition 3.5.1. The optimal solution of the problem (3.56)-(3.60) is $\alpha^{*}=2$.

Proof. It is easy to see that when all the lower bounds have the same value $L$, and the upper bounds have the same value $U$, then $-l(i, j)=2(U-L)^{2}$. Considering the problem of maximizing $\alpha$, with the constraints $\forall i<j \leq n \quad \alpha \leq 2(U-L)^{2}$, the objective function value of the optimal solution is $\alpha^{*}=2(U-L)^{2}$. Since $L=0$ and $U=1$, then $\alpha^{*}=2$.

The bound provided by the previous relaxation is roughly loose: since $\alpha$ is the square of the minimum distance between the points, the upper bound on the distance is $\sqrt{2}$, that is the cost of the optimal solution obtained when there are only 2 points in the square, placed in two opposite vertices. Furthermore, this bound does not depend on the number of points $n$, nor on the value of the variables $x$ and $y$ : due to the fact that all the lower and upper bounds have the same value, in the linear relaxation $l(i, j)$ all the coefficients of the terms containing $x$ and $y$ become 0 .

In order to improve the value of $\alpha^{*}$, we should change the value of lower and upper bounds for some variables; thus, the corresponding terms containing $x$ and $y$ in the linear relaxation do not disappear. The following conjecture refers to that idea.

Conjecture 3.5.2. Consider an instance of PPS with $n$ points. Divide the unit square in $k^{2}$ equal subsquares, with $k=\arg \min \left|\frac{n}{2}-s^{2}\right|, s \in\left\{\left\lceil\sqrt{\frac{n}{2}}\right\rceil,\left\lfloor\sqrt{\frac{n}{2}}\right\rfloor\right\}$. There is at least one point of the optimal solution in each subsquare.

The meaning of this conjecture is that we can change the value of the bounds for $k^{2}$ points. For example, consider the case with $n=9$ : here, $k=2$, so there are 4 


\begin{tabular}{|c|c|c|c|c|c|}
\hline \multirow[b]{2}{*}{$n$} & \multirow[b]{2}{*}{$m^{*}$} & \multicolumn{2}{|c|}{ Original formulation } & \multicolumn{2}{|c|}{ bound constraints formulation } \\
\hline & & LB & UB & LB & UB \\
\hline 9 & 0.5 & 0.000098 & 1.414213 & 0.300463 & 0.707107 \\
\hline 10 & 0.421279 & 0.000098 & 1.414213 & 0.396156 & 0.707107 \\
\hline 11 & 0.398207 & 0.000099 & 1.414213 & 0.000099 & 0.707107 \\
\hline 12 & 0.388730 & 0.000099 & 1.414213 & 0.360065 & 0.707107 \\
\hline 13 & 0.366096 & 0.000098 & 1.414213 & 0.339654 & 0.502948 \\
\hline 14 & 0.348915 & 0.000098 & 1.414213 & 0.340830 & 0.502874 \\
\hline 15 & 0.341081 & 0.000098 & 1.414213 & 0.334524 & 0.502793 \\
\hline 16 & 0.333333 & 0 & 1.414213 & 0.290033 & 0.502793 \\
\hline 17 & 0.306153 & 0 & 1.414213 & 0.000099 & 0.502793 \\
\hline 18 & 0.300462 & 0 & 1.414213 & 0.252819 & 0.502793 \\
\hline 19 & 0.289541 & 0.000047 & 1.414213 & 0.252337 & 0.502793 \\
\hline 20 & 0.286611 & 0 & 1.414213 & 0.276468 & 0.502793 \\
\hline
\end{tabular}

Table 3.7: Results obtained at the root node of the sBB tree by COUEnNE for some instances of PPS with and without range tightening of the variables.

subsquares. According to the conjecture, we can place one point in each subsquare; for instance, if we put the point $i$ is in the bottom left subsquare, we can modify the bounds provided by (3.8) obtaining $x_{i} \leq 0.5$ and $y_{i} \leq 0.5$.

In order to change other bounds, we can use the $x$ bound constraints and the $y$ bound constraints presented in Section 3.4.2. After dividing the square in $k^{2}$ subsquares, we have placed in the left half of the square $\eta<n_{x}$ points, so for other $n_{x}-\eta$ points we can change the upper bounds on the coordinates $x$ from 1 to 0.5 , according to the $x$ bound constraints. A similar idea can be used for the $y$ bound constraints.

Table 3.7 presents the values of the upper and lower bounds for some instances of the PPS problem obtained at the root node of the sBB of CouEnne, with and without the constraints derived from Conjecture 3.5.2. The value of the upper bound is obtained by solving a linear relaxation of the problem, whereas the lower bound is the best solution found so far. The values of the optimal distance $d^{*}=\sqrt{\alpha^{*}}$ are also reported (they can be found in http://www.packomania.com and in [237]).

The results presented in Table 3.7 show that using the range tightening constraints the upper bounds are better, as well as the lower bounds which in some cases provide solutions not far from the optimal ones. Moreover, we can see an improvement of the upper bounds from the instance $n=12$ (where $k=3$ ) to the instance $n=13$ (where $k=4$ ). The fact that without these constraints the quality of the solution at the root node is poor can be seen as another side effect of symmetries, since having the upper (lower) bounds equal for all the variables yields a bad linear relaxation of the problem, as show by Proposition 3.5.1.

In order to extend this result to the PECS problem, one should divide the square $[r, 1-r]^{2}$ in $k^{2}$ subsquares, instead of dividing the $[0,1]^{2}$ square as done for PPS (it is more clear if looking at Figure 3.1). For example, if $k=3$ we obtain 9 subsquares of 
side $\frac{1-2 r}{3}$. Then, the center of the circle $i$ which is inside the left bottom subsquare has $x_{i} \in\left[r, r+\frac{1-2 r}{3}\right]$ and $y_{i} \in\left[r, r+\frac{1-2 r}{3}\right]$.

\subsection{Conclusions}

In this chapter we presented the problem of packing equal circles in a square as example of optimization problem involving a high degree of symmetries. The presence of symmetric optima is a problem for sBB algorithms, since the BB tree becomes large and the time to reach the leaves, i.e., the optimal solutions, increases. In order to make some of the symmetric optima infeasible we proposed some reformulations of the original model. In the first part of the chapter we presented different formulations for the problem of packing equal circles in a square (i.e., PECS and PPS), and we show some numerical results which support our decision to employ the PECS formulation. Then, we introduced three classes of SBCs, called weak, strong, and mixed, which yield narrowing reformulations. The mixed SBCs based formulation was the most effective to remove symmetries, and it provides good quality solution already at the root node of the BB tree. In the second part of the chapter we proposed some other inequalities to tighten the formulation. Starting from the PECS formulation with strong SBCs, we derived a new formulation which can be solved faster. We used strong SBCs and not mixed SBCs because some of these new inequalities needed the strong SBCs to be valid.

In the last part of the chapter we presented a conjecture about the reduction of the variables range. We used the PPS formulation in this case, since it is easier to describe the conjecture, but we extended also the result for PECS. Basically, we stated that the unit square can be divided in a number of subsquares that is close to half of the number of points $n$ of the PPS instance, and that in the optimal solution each subsquare contains at least a point. Looking at the optimal and best known solutions this conjecture seems always to be valid (furthermore, it seems the number of subsquares can also be increased). The effect of this conjecture is that the new tight ranges of the variables associated to the points which belong to these subsquares yield to both a better upper bound and a better incumbent already at the root node of the sBB tree. The effect of having bad quality solutions at the root node was also observed when introducing SBCs, and the results were better with the SBCs-based formulations.

Even if we did not improve the best known solutions (in terms of value of the objective function), this chapter in interesting to show the effect of symmetries in MP and some techniques to remove them. However, the best results found in the literature for PECS are obtained mostly by heuristics and specifically designed algorithms. Albeit we were able to remove all the symmetries, the problem would still be hard because it is nonlinear and nonconvex, and sBB based algorithms would not be able to solve large instances. 


\section{Part III}

\section{An application of relaxations}



This part of the thesis concerns the comparison of two methods, called respectively primal and dual, to represent the convex relaxations for multilinear terms. More precisely, the primal relaxation consists of replacing each multilinear term with a new variable, and a set of constraints to be adjoined to the model. The dual relaxation is obtained using the dual representation of the convex (lower)/concave (upper) envelopes associated to each multilinear term, i.e., the convex combination of their extreme points using dual variables $\lambda$. The theory underlying these relaxations is well-known, hence the contribution of this chapter is not theoretical. Rather, we present a computational analysis which shows that the dual approach leads to a formulation that is easier to write, is more stable, and that can be solved faster with respect to the model obtained using the primal approach, when the number of multilinear terms increases. Moreover, the primal relaxation can be written in an optimal way (in some sense) only for bilinear and trilinear problems, and partly for quadrilinear problems, whereas the dual approach can be used for any multilinear term. This work has been presented in [62]. 
$\Gamma_{\text {Chapter }}$

\section{Primal and dual convex relaxations for multilinear terms}

Several problems in the literature are described by means of MP models where products of $k$ variables $x_{1} \cdots x_{k}$ can appear in the constraints and in the objective function. The corresponding term is called bilinear if $k=2$, trilinear if $k=3$, quadrilinear if $k=4$, and so on. In general, they are called multilinear terms.

Among the most well-known applications involving multilinear terms, there are pooling and blending problems $[4,14,67,96,124,191]$, where bilinear products occur whenever $x_{1}$ indicates a percentage and $x_{2}$ an oil flow in a pipe. The Hartree-Fock problem [163] minimizes a quartic energy expression (involving quadrilinear terms) subject to some orthogonality constraints (involving bilinear terms). The molecular distance geometry problem [164] involves bilinear or quadrilinear terms depending on which formulation is used. General multilinear terms involving continuous variables occur in multilinear least-squares problems [202]. In general, such products occur over bounded variables: most applications require the variables $x=\left(x_{1}, \ldots, x_{k}\right)$ to be bounded to the hyperrectangle $\left[x^{L}, x^{U}\right]$, where $x^{L}=\left(x_{1}^{L}, \ldots, x_{k}^{L}\right)$ and $x^{U}=$ $\left(x_{1}^{U}, \ldots, x_{k}^{U}\right)$. We remark, however, that there exists an application from code debugging $[109,165]$ exhibiting bilinear terms $x_{1} x_{2}$ where $x_{1} \in\{0,1\}$ and $x_{2}$ must be unbounded for the model to be correct (such variables are used to ensure that loops terminate whenever no upper bound is explicitly known for the loop counter).

Since the models having multilinear terms are nonconvex and nonlinear, one must employ sBB algorithms in order to obtain a guaranteed solution. sBB methods employ a convex relaxation of the problem at each search node. Such relaxations can be obtained in two ways: the traditional method consists of representing the convex hull (defined by convex and concave envelopes) by means of a set of inequalities to adjoin to the original model. One can alternatively use the dual representation of these envelopes, i.e., the convex combinations of the extreme points of the convex hull using dual variables $\lambda$. In the remainder, the former method is called primal 
relaxation, while the latter is referred as dual relaxation. The inequalities needed for the primal relaxation are known explicitly for the bilinear case, the trilinear case, and some cases of the quadrilinear case. On the other hand, the dual relaxation needs more variables, fewer constraints, and no special case-by-case treatment. Moreover, we show that the relaxation obtained using duality performs more efficiently than the traditional (primal) method. Note that the optimal solution provided by the primal and dual formulations are the same.

The rest of this chapter is organized as follows: Section 4.1 reports definitions and notation useful to understand the following sections. In Section 4.2 the primal relaxation method is presented. More precisely, the inequalities for the bilinear case (and partly for the trilinear case) are reported. For the quadrilinear case the number of constraints is too large, and for higher dimensions the constraints are not known explicitly. In Section 4.3 the dual relaxation method is introduced. Then, in Section 4.4 a comparison between the two relaxation methods on some test instances is presented. Finally, in Section 4.5 conclusions are drawn.

\subsection{Definitions and notation}

Let $S \subseteq \mathbb{R}^{n}$ be non-empty. Any convex set containing $S$ is a convex relaxation of $S$. The convex hull of $S$ is the intersection of all convex relaxations of $S$. A graphical representation in given in Figure 4.1.
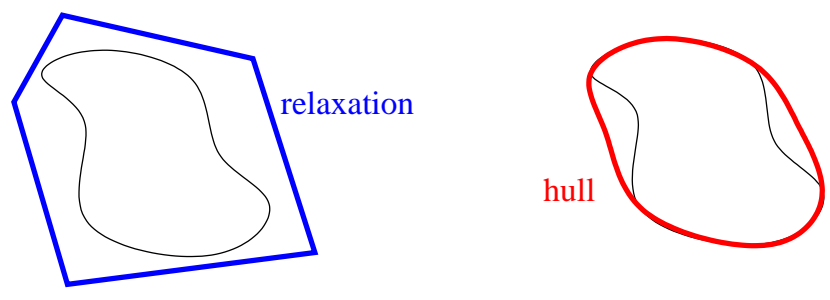

Figure 4.1: Convex relaxation and convex hull of the set $S$.

Let $C \subseteq \mathbb{R}^{n}$ be compact (i.e., closed and bounded) and convex, and $f: C \rightarrow \mathbb{R}$ be lower semicontinuous (i.e., there is a point $x_{d}$ such that in the neighborhood of $x_{d}$ the function value is either close to $f\left(x_{d}\right)$ or greater than $\left.f\left(x_{d}\right)\right)$. Any convex function underestimating $f$ is a convex relaxation of $f$. The convex envelope of $f$ is the pointwise supremum of all convex underestimators of $f$. This is shown in Figure 4.2 .

The general multilinear term is given by:

$$
w(x)=x_{1} \cdots x_{k}
$$

for some $k \in \mathbb{N}$, and is possibly the most common nonlinear term occurring naturally in MP applications. If the $k$ indices are taken from a larger set, we might also write 

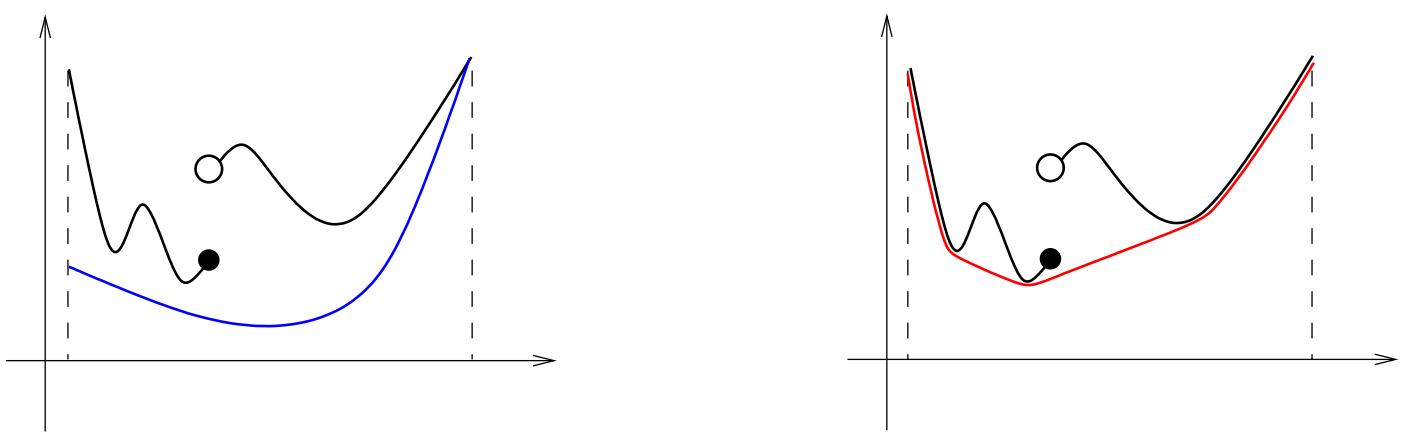

Figure 4.2: Convex relaxation and convex envelope of the function $f$.

(4.1) as $w(x)=x_{j_{1}} \cdots x_{j_{k}}$ with $J=\left\{j_{1}, \ldots, j_{k}\right\}$. We define the set $W_{J}$ as:

$$
W_{J}=\left\{\left(x, w_{J}\right) \mid w_{J}=\prod_{j \in J} x_{j} \wedge x \in\left[x^{L}, x^{U}\right]\right\}
$$

We let $P$ be the set of vertices of the hyperrectangle $\left[x^{L}, x^{U}\right]$ and $P_{W}$ be the lifting of $P$ in the space spanned by $\left(x, w_{J}\right)$, where, for each point $\bar{x} \in P$, the corresponding point in $P_{W}$ is obtained by setting $w_{J}=w(\bar{x})$. The convex hull of the set $W_{J}$ is defined as:

$$
\breve{W}_{J}=\left\{\left(x, w_{J}\right) \mid w_{J} \geq \hat{w}(x) \wedge w_{J} \leq \check{w}(x) \wedge x \in\left[x^{L}, x^{U}\right]\right\}
$$

where $\hat{w}(x)$ and $\breve{w}(x)$ are respectively the convex and concave envelopes of the multilinear term. With a slight abuse of notation, the constraints on $w_{J}$ appearing in the definition of $\breve{W}_{J}$ are also called convex envelopes of the multilinear terms. However, in problems involving several multilinear terms, the set of all the convex envelopes associated to each multilinear term does not represent the convex hull associated to the feasible region of the problem, but only a convex relaxation.

It was shown in [215] that the convex envelopes of multilinear terms are vertex polyhedral [238], i.e., $\breve{W}_{J}$ is a polyhedron having $P_{W}$ as vertex set. This makes it possible to write the convex envelopes of multilinear terms by means of linear constraints, yielding the primal relaxation method, presented in the next section. As recalled in Section 1.2.1.1, linear inequalities define a convex set, thus the corresponding relaxation is a convex problem.

\subsection{Primal relaxation}

For the general case, convex envelopes for multilinear terms are available explicitly in function of $x^{L}, x^{U}$ for $k \in\{2,3\}$ and partly for $k=4$. As stated earlier, such envelopes consist of sets of constraints to be adjoined to the MP formulation.

Whenever $x \in\left[x^{L}, x^{U}\right]$ and at least $k-1$ variables out of $k$ are constrained 
to be integer, the corresponding multilinear term can be linearized exactly. Each general integer variable is replaced by an aggregation of binary variables (for example choosing the value taken by the original integer variable), and the original multilinear term $w(x)$ is replaced by a sum of multilinear terms with at least $k-1$ binary variables. A sequence of $k-1$ Fortet's linearizations (see Section 4.2.1.2) will then yield a MILP formulation of the original multilinear term.

Whenever at least two variables in a multilinear term are continuous, exact linearizations are in general no longer possible, and one must resort to solution techniques for nonconvex programs, such as the sBB algorithm presented in Section 1.2.2.5. This involves repeatedly solving the original problem and a convex relaxation thereof over appropriate sets of ranges $\left[x^{L}, x^{U}\right]$. The relaxation is obtained by replacing each multilinear term with an added variable $w_{J}$ and adjoining some constraints to the formulation which define a convex relaxation of $W_{J}$. In general, the tighter these relaxations are, the more efficient the sBB will be.

\subsubsection{Bilinear terms}

Consider now a problem having a bilinear term. For the sake of clarity, we suppose that the two variables involved in the product are $x_{1}$ and $x_{2}$, and the product is $w\left(x_{1}, x_{2}\right)=x_{1} x_{2}$. A graphical representation of the surface $w\left(x_{1}, x_{2}\right)$ is shown in Figure 4.3.

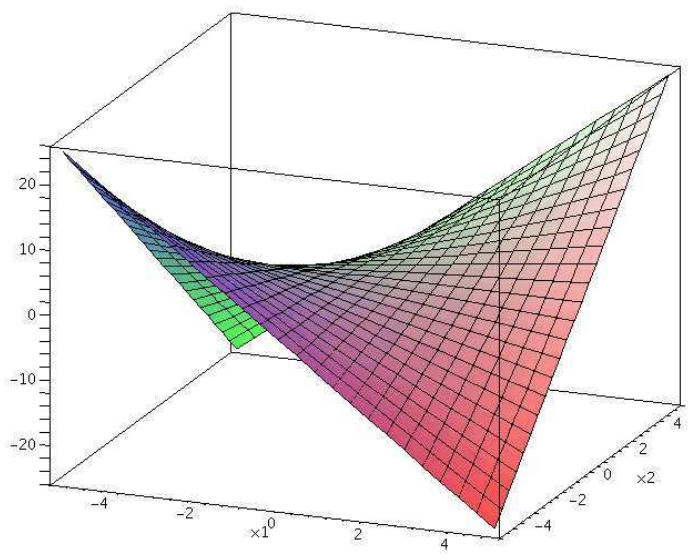

Figure 4.3: The bilinear surface $w\left(x_{1}, x_{2}\right)=x_{1} x_{2}$. 


\subsubsection{McCormick's inqualities}

The constraints used to define the convex envelopes for the term $x_{1} x_{2}$ are the following:

$$
\begin{aligned}
& w_{1,2} \geq x_{1}^{L} x_{2}+x_{2}^{L} x_{1}-x_{1}^{L} x_{2}^{L} \\
& w_{1,2} \geq x_{1}^{U} x_{2}+x_{2}^{U} x_{1}-x_{1}^{U} x_{2}^{U} \\
& w_{1,2} \leq x_{1}^{L} x_{2}+x_{2}^{U} x_{1}-x_{1}^{L} x_{2}^{U} \\
& w_{1,2} \leq x_{1}^{U} x_{2}+x_{2}^{L} x_{1}-x_{1}^{U} x_{2}^{L},
\end{aligned}
$$

where $w_{1,2}$ is the new variable replacing the product $x_{1} x_{2}$. These constraints, called McCormick inequalities, were first described in [183] and later shown to be envelopes in [9]. Figure 4.4 shows the lower convex and upper concave envelopes for the bilinear term $x_{1} x_{2}$. The former is defined by constraints (4.2) and (4.3), while the latter is defined by (4.4) and (4.5).
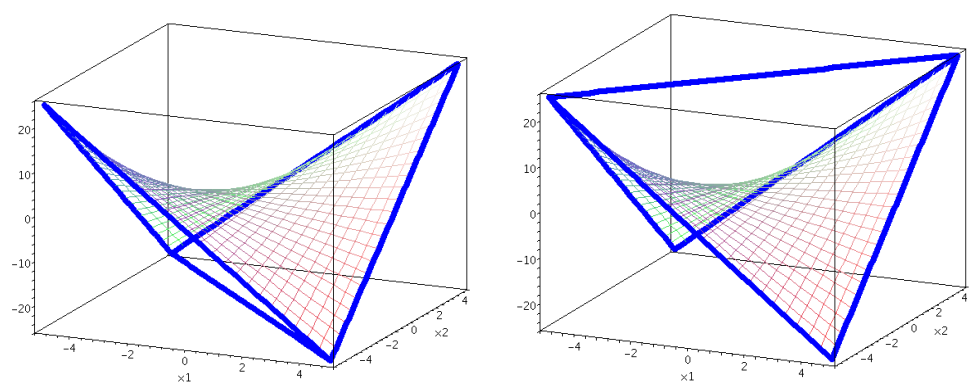

Figure 4.4: Lower convex (left) and upper concave (right) envelopes for the bilinear term.

\subsubsection{Fortet inequalities}

It was observed in $[93,119]$ that if $k=2$ and $x_{1}, x_{2} \in\{0,1\}$, then $w(x)$ can be replaced by an added variable $w_{1,2} \in[0,1]$ whilst the Fortet inequalities are adjoined to the model:

$$
\begin{aligned}
& w_{1,2} \geq 0 \\
& w_{1,2} \geq x_{1}+x_{2}-1 \\
& w_{1,2} \leq x_{1} \\
& w_{1,2} \leq x_{2},
\end{aligned}
$$

which can be obtained by the McCormick inequalities where $x_{1}^{L}=x_{2}^{L}=0$ and $x_{1}^{U}=x_{2}^{U}=1$. This reformulation is an exact linearization of the original bilinear program $[157,160]$ (i.e., $w_{1,2}=1$ if and only if $x_{1}=1$ and $x_{2}=1$ ). 


\subsubsection{Trilinear terms: Meyer-Floudas inequalities}

Significant progress was made by Meyer and Floudas [187, 188], who were able to write the explicit envelopes for the trilinear term $w(x)=x_{1} x_{2} x_{3}$. Their exact form depends on the relative sign of the variable bounds $x^{L}, x^{U}$. The cases where the bound signs are equal are discussed in [187] (each case giving rise to 12 inequalities), whereas the cases where the bounds have opposite signs for at least one variable are discussed in [188]. Several of these cases also involve checking nontrivial bound relations. Although Meyer and Floudas' results are conceptually simple to apply (it suffices to establish which is the case at hand, and adjoin the corresponding inequalities to the MP), the inequalities themselves are much more involved than McCormick's, and it is very easy to make mistakes when integrating them in a computer program. Worst of all, however, is the fact that some coefficients appearing in Meyer-Floudas inequalities involve nontrivial floating point operations. For example, see the coefficients $c_{1}$ and $c_{2}$ of $x_{1}$ in (4.6) and (4.7). As is well-known, floating point additions and subtractions are error-prone [141, 4.2.1]. This will yield an inaccurate constraint representation of $\breve{W}_{J}$; to make things worse, as the simplex method will identify optimal solutions at the vertices of the polyhedron, then this inaccuracy will impact the optimal solution. In particular, if variables are constrained to be integer, a feasible integer solution on or near the vertex of the polyhedron might be deemed infeasible. By contrast, each coefficient of the the McCormick inequalities $(k=2)$ only involves floating point multiplication, which is a much safer operation.

As example, we report the Meyer-Floudas inequalities for the case where the three variables involved in the product have nonnegative lower (and upper) bounds [187]. To write the convex envelope, first the three variables must be mapped onto the variables $x_{1}, x_{2}$, and $x_{3}$ such that the following relationships hold:

$$
\begin{aligned}
& x_{1}^{U} x_{2}^{L} x_{3}^{L}+x_{1}^{L} x_{2}^{U} x_{3}^{U} \leq x_{1}^{L} x_{2}^{U} x_{3}^{L}+x_{1}^{U} x_{2}^{L} x_{3}^{U} \\
& x_{1}^{U} x_{2}^{L} x_{3}^{L}+x_{1}^{L} x_{2}^{U} x_{3}^{U} \leq x_{1}^{U} x_{2}^{U} x_{3}^{L}+x_{1}^{L} x_{2}^{L} x_{3}^{U} .
\end{aligned}
$$

Then, the following constraints (defining the convex envelope) have to be adjoined to the model:

$$
\begin{aligned}
& w_{1,2} \geq x_{2}^{L} x_{3}^{L} x_{1}+x_{1}^{L} x_{3}^{L} x_{2}+x_{1}^{L} x_{2}^{L} x_{3}-2 x_{1}^{L} x_{2}^{L} x_{3}^{L} \\
& w_{1,2} \geq x_{2}^{U} x_{3}^{U} x_{1}+x_{1}^{U} x_{3}^{U} x_{2}+x_{1}^{U} x_{2}^{U} x_{3}-2 x_{1}^{U} x_{2}^{U} x_{3}^{U} \\
& w_{1,2} \geq x_{2}^{L} x_{3}^{U} x_{1}+x_{1}^{L} x_{3}^{U} x_{2}+x_{1}^{U} x_{2}^{L} x_{3}-x_{1}^{L} x_{2}^{L} x_{3}^{U}-x_{1}^{U} x_{2}^{L} x_{3}^{U} \\
& w_{1,2} \geq x_{2}^{U} x_{3}^{L} x_{1}+x_{1}^{U} x_{3}^{L} x_{2}+x_{1}^{L} x_{2}^{U} x_{3}-x_{1}^{U} x_{2}^{U} x_{3}^{L}-x_{1}^{L} x_{2}^{U} x_{3}^{L} \\
& w_{1,2} \geq c_{1} x_{1}+x_{1}^{U} x_{3}^{L} x_{2}+x_{1}^{U} x_{2}^{L} x_{3}+x_{1}^{L} x_{2}^{U} x_{3}^{U}-c_{1} x_{1}^{L}-x_{1}^{U} x_{2}^{U} x_{3}^{L}-x_{1}^{U} x_{2}^{L} x_{3}^{U} \\
& w_{1,2} \geq c_{2} x_{1}+x_{1}^{L} x_{3}^{U} x_{2}+x_{1}^{L} x_{2}^{U} x_{3}+x_{1}^{U} x_{2}^{L} x_{3}^{L}-c_{2} x_{1}^{U}-x_{1}^{L} x_{2}^{L} x_{3}^{U}-x_{1}^{L} x_{2}^{U} x_{3}^{L},
\end{aligned}
$$


where

$$
\begin{aligned}
c_{1} & =\frac{x_{1}^{U} x_{2}^{U} x_{3}^{L}-x_{1}^{L} x_{2}^{U} x_{3}^{U}-x_{1}^{U} x_{2}^{L} x_{3}^{L}+x_{1}^{U} x_{2}^{L} x_{3}^{U}}{x_{1}^{U}-x_{1}^{L}} \\
c_{2} & =\frac{x_{1}^{L} x_{2}^{L} x_{3}^{U}-x_{1}^{U} x_{2}^{L} x_{3}^{L}-x_{1}^{L} x_{2}^{U} x_{3}^{U}+x_{1}^{L} x_{2}^{U} x_{3}^{L}}{x_{1}^{L}-x_{1}^{U}} .
\end{aligned}
$$

The concave envelope is defined by these constraints:

$$
\begin{aligned}
& w_{1,2} \leq x_{2}^{L} x_{3}^{L} x_{1}+x_{1}^{U} x_{3}^{L} x_{2}+x_{1}^{U} x_{2}^{U} x_{3}-x_{1}^{U} x_{2}^{U} x_{3}^{L}-x_{1}^{U} x_{2}^{L} x_{3}^{L} \\
& w_{1,2} \leq x_{2}^{U} x_{3}^{L} x_{1}+x_{1}^{L} x_{3}^{L} x_{2}+x_{1}^{U} x_{2}^{U} x_{3}-x_{1}^{U} x_{2}^{U} x_{3}^{L}-x_{1}^{L} x_{2}^{U} x_{3}^{L} \\
& w_{1,2} \leq x_{2}^{L} x_{3}^{L} x_{1}+x_{1}^{U} x_{3}^{U} x_{2}+x_{1}^{U} x_{2}^{L} x_{3}-x_{1}^{U} x_{2}^{L} x_{3}^{U}-x_{1}^{U} x_{2}^{L} x_{3}^{L} \\
& w_{1,2} \leq x_{2}^{U} x_{3}^{U} x_{1}+x_{1}^{L} x_{3}^{L} x_{2}+x_{1}^{L} x_{2}^{U} x_{3}-x_{1}^{L} x_{2}^{U} x_{3}^{U}-x_{1}^{L} x_{2}^{U} x_{3}^{L} \\
& w_{1,2} \leq x_{2}^{L} x_{3}^{U} x_{1}+x_{1}^{U} x_{3}^{U} x_{2}+x_{1}^{L} x_{2}^{L} x_{3}-x_{1}^{U} x_{2}^{L} x_{3}^{U}-x_{1}^{L} x_{2}^{L} x_{3}^{U} \\
& w_{1,2} \leq x_{2}^{U} x_{3}^{U} x_{1}+x_{1}^{L} x_{3}^{U} x_{2}+x_{1}^{L} x_{2}^{L} x_{3}-x_{1}^{L} x_{2}^{U} x_{3}^{U}-x_{1}^{L} x_{2}^{L} x_{3}^{U} .
\end{aligned}
$$

\subsubsection{Quadrilinear terms}

For quadrilinear terms, the explicit envelopes have not been found yet. A first attempt in this direction is presented in the M.Sc. thesis of S. Balram [18], where 44 inequalities for the simplest of the quadrilinear cases (all bounds in the nonnegative orthant) are presented. The thesis does not mention how many cases there will be in total for $k=4$, but several coefficients of this simplest case involve even more floating point additions and subtractions than the Meyer-Floudas' inequalities, and are therefore expected to yield inaccurate formulations. As for the trilinear case, when integer variables are involved, some feasible solutions might be incorrectly deemed infeasible.

Another way to relax quadrilinear terms is to employ McCormick and MeyerFloudas relaxations. The associative expression for $x_{1} x_{2} x_{3} x_{4}$ yielding the tightest convex relaxation is obtained by combining the convex envelope of trilinear terms and that of bilinear terms $[25,49]$.

The state-of-the-art in computing envelopes for multilinear terms with $k \geq 3$ involves the use of PORTA [55] (which implements the Fourier-Motzkin elimination algorithm [70], and given specific values for $x^{L}, x^{U}$, is able to write the corresponding constraints for the envelopes of the points in $P_{W}$ ) or cdd [100]. Since the resulting inequalities change in function of the bounds, the use of this software within the sBB algorithm, where the bounds change at each node, would be prohibitive.

\subsection{Dual relaxation}

The fact that the envelopes of multilinear terms are vertex polyhedral immediately suggests the following dual approach: express a point in $\breve{W}_{J}$ as the convex combina- 
tion of the set $P_{W}$ of extreme points of $\breve{W}_{J}$. We look for a vector $\lambda$ of $2^{k}$ nonnegative Lagrange multipliers such that:

$$
[x, w]=\sum_{i \leq 2^{k}} \lambda_{i} p_{i} \wedge \sum_{i \leq 2^{k}} \lambda_{i}=1
$$

where $P_{W}=\left\{p_{1}, \ldots, p_{2^{k}}\right\} \subseteq \mathbb{R}^{k+1}$. Now all that remains to do, in order to make (4.3) explicit envelopes, is to express the $p_{i}$ 's in function of $x^{L}, x^{U}$. To this aim, we define two elements: the binary $2^{k} \times k$ matrix $D=\left(d_{i, j}\right)$, and the function $b$ which maps a binary vector of dimension $k$ to a vector of the same dimension such that a value of 0 (1) in position $j$ of the input vector corresponds to the lower (upper) bound of the variable $x_{j}$ in the position $j$ of the output vector. Each row $i$ of $D$ is the binary representation of the integer number $i-1$ (since $i$ starts from 1 ) using $k$ digits. In this way $d_{i j}$ is either 0 or 1 according as to whether the $j$-th component of $p_{i}$ is a lower or upper bound, and $b_{j}\left(d_{i j}\right)$ returns the correct component:

$$
\forall j \leq k \quad b_{j}(0)=x_{j}^{L} \wedge b_{j}(1)=x_{j}^{U}
$$

We relax the $k$-linear term $w(x)=x_{1} \cdots x_{k}$ as follows. We add $2^{k}$ new nonnegatively constrained variables $\lambda_{i} \geq 0$ (for $i \leq 2^{k}$ ) and $k+2$ new constraints:

$$
\begin{aligned}
& \forall j \leq k \quad x_{j}=\sum_{i \leq 2^{k}} \lambda_{i} b_{j}\left(d_{i j}\right) \\
& w=\sum_{i \leq 2^{k}} \lambda_{i} \prod_{j \leq k} b_{j}\left(d_{i j}\right) \\
& \sum_{i \leq 2^{k}} \lambda_{i}=1 .
\end{aligned}
$$

Let $\bar{W}_{J}=\{(x, w, \lambda) \mid(4.8)-(4.10) \wedge \lambda \geq 0\}$. By geometry and duality in LP, the projection of $\bar{W}_{J}$ on the $(x, w)$ variables is precisely $\breve{W}_{J}$.

\subsubsection{Example}

To better explain the dual method, suppose we want to relax the bilinear term $x_{1} x_{2}$ by replacing it with the variable $w_{1,2}$, where $x_{1} \in\left[x_{1}^{L}, x_{1}^{U}\right]$ and $x_{2} \in\left[x_{2}^{L}, x_{2}^{U}\right]$.

The matrix $D$ is the following:

$$
\left[\begin{array}{ll}
0 & 0 \\
0 & 1 \\
1 & 0 \\
1 & 1
\end{array}\right]
$$


Applying the function $b$ we obtain:

$$
\left[\begin{array}{cc}
x_{1}^{L} & x_{2}^{L} \\
x_{1}^{L} & x_{2}^{U} \\
x_{1}^{U} & x_{2}^{L} \\
x_{1}^{U} & x_{2}^{U}
\end{array}\right]
$$

Hence, equations (4.8)-(4.10) become:

$$
\begin{aligned}
& x_{1}=\lambda_{1} x_{1}^{L}+\lambda_{2} x_{1}^{L}+\lambda_{3} x_{1}^{U}+\lambda_{4} x_{1}^{U} \\
& x_{2}=\lambda_{1} x_{2}^{L}+\lambda_{2} x_{2}^{U}+\lambda_{3} x_{2}^{L}+\lambda_{4} x_{2}^{U} \\
& w_{1,2}=\lambda_{1} x_{1}^{L} x_{2}^{L}+\lambda_{2} x_{1}^{L} x_{2}^{U}+\lambda_{3} x_{1}^{U} x_{2}^{L}+\lambda_{4} x_{1}^{U} x_{2}^{U} \\
& \sum_{i \leq 4} \lambda_{i}=1,
\end{aligned}
$$

where $\forall i \in\{1,2,3,4\} \quad \lambda_{i} \geq 0$.

\subsection{Comparison and numerical results}

Our tests, carried out on an Intel Xeon CPU at 2.66GHz with 24GB RAM, show that dual relaxations can be solved faster (as the formulation size increases) than primal relaxations, and are also more stable. We measure speed by simply solving the primal and dual relaxations for the same original problem using CPLEX 12.2 [135], and comparing CPU times. We define a method stable when its CPU time increase looks empirically proportional to the increase in formulation size. Firstly we consider NLP problems, and we solve the corresponding primal LP relaxation and dual LP relaxation. Then we measure stability by enforcing integrality constraints on some of the problem variables, obtaining MINLPs: this yields a primal MILP relaxation and a dual MILP relaxation. Both are solved with CPLEX 12.2, and the CPU times are recorded and compared. This is meant to simulate the behavior of these relaxations in a $\mathrm{BB}$ setting. It turns out that the running times of the MILP solver on the dual MILP relaxation is proportional to the relaxation size, whereas it varies wildly for the primal MILP relaxation.

We generated 2520 random multilinear nonseparable NLPs, involving linear, bilinear, and trilinear terms. For each such NLP $P$, we generated the primal LP relaxation $R_{P}$ and the dual LP relaxation $\Lambda_{P}$. Then we set some variables of the previously generated NLPs to be integer, thus obtaining MINLPs, and for each MINLP $P$, we generated the primal MILP relaxation $R_{P}^{\prime}$ and the dual MILP relaxation $\Lambda_{P}^{\prime}$. We let $n$ (the number of original variables) vary in $\{10,20\}$. For $n=10$ we let the number of bilinear terms $\beta$ vary in $\{0,10,13,17,21,25,29,33\}$ and of trilinear terms $\tau$ in $\{0,10,22,34,36,58,71,83\}$. For $n=20$, we let $\beta$ vary in $\{0,20,38,57,76,95,114,133\}$ and $\tau$ in $\{0,20,144,268,393,517,642,766\}$. Note 
that the total number of combinations of $(n, \beta, \tau)$, given $n$, is 63 , because the case $\beta=\tau=0$ is excluded. For each combination of the triplet $(n, \beta, \tau)$ we generated 20 random instances. In summary, we have 63 combinations for each value of $n$, and we have two values of $n$ (that is, 10 and 20) For each case we generated 20 random instances, and we obtain a total of $63 \cdot 2 \cdot 20=2520$ random instances. The variable bounds, chosen at random, were all of magnitude $\pm 1 \times 20$.

The CPU time results (in seconds) comparing $R_{P}, \Lambda_{P}$ are given in Figures 4.54.6. The horizontal axis is marked by the instance ID. Each recognizable "block" corresponds to a fixed value of $\beta$. Since bilinear terms give rise to fewer relaxation variables/constraints than trilinear ones, the formulation size is strongly proportional to $\tau$ and weakly proportional to $\beta$. The CPU time results (in seconds) comparing $R_{P}^{\prime}, \Lambda_{P}^{\prime}$ are given in Figures 4.7-4.8.

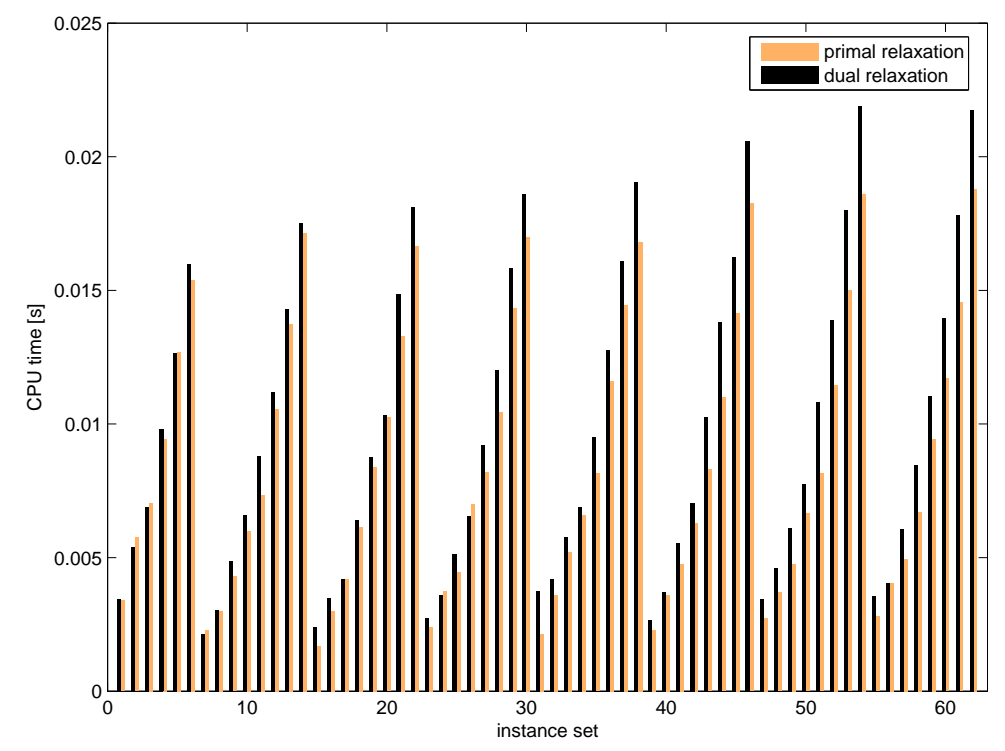

Figure 4.5: CPU time averages (in seconds) over each 20-instance set with given $(n, \beta, \tau)$ with $n=10$ for the LP relaxations.

\subsection{Conclusions}

From the cases $k=3$ and $k=4$ it appears clearly that the explicit form of the inequalities describing $\breve{W}_{J}$, in function of $x^{L}, x^{U}$, considerably increases in complexity (from the point of view of floating point additions and subtractions) as $k$ increases, thereby causing numerical instability. But this is not all: the number of such inequalities, even when they are found explicitly with PORTA, also increases, thereby yielding ever more sizable formulations. While it is known that this number increases as $O\left(2^{k}\right)$, the first column of Table 4.1 suggests that the increase is more 


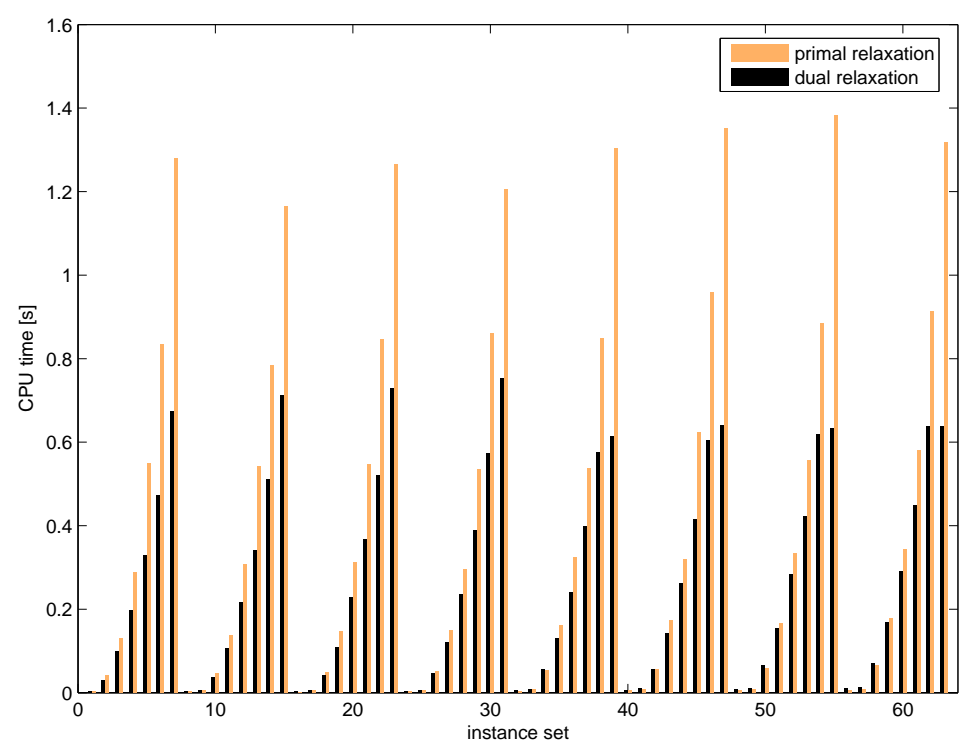

Figure 4.6: CPU time (in seconds) averages over each 20-instance set with given $(n, \beta, \tau)$ with $n=20$ for the LP relaxations.

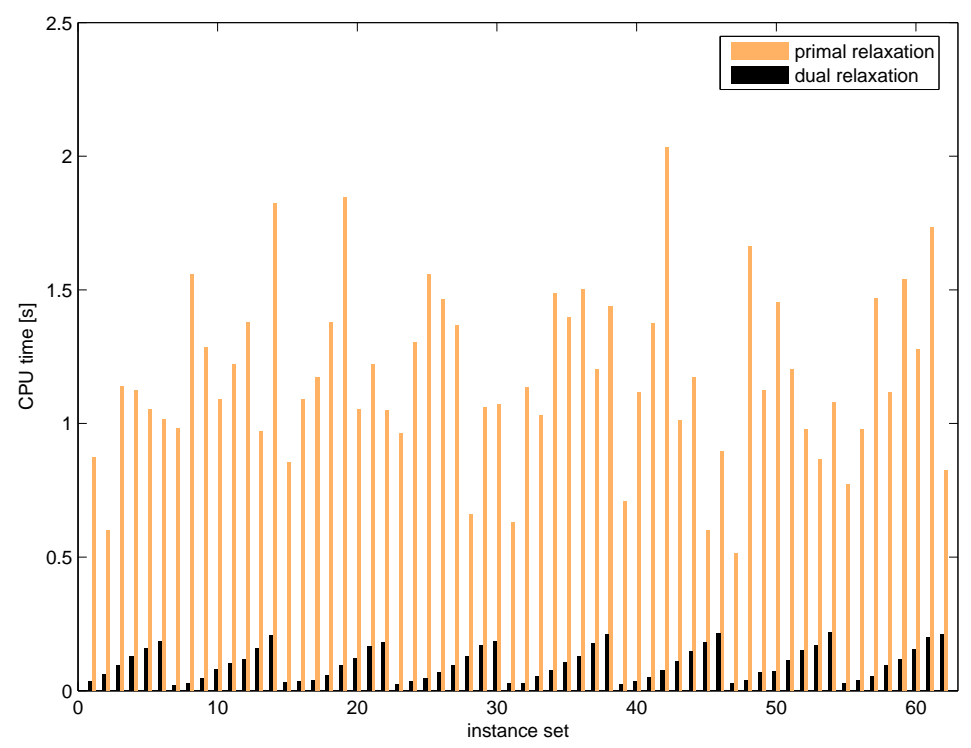

Figure 4.7: CPU time averages (in seconds) over each 20-instance set with given $(n, \beta, \tau)$ with $n=10$ for the MILP relaxations.

like $O\left(k 2^{k}\right)$.

On the other hand, the dual envelope adds exactly $2^{k}$ new nonnegative variables and $k+2$ new constraints to the formulation. Table 4.1 reports the size increases for the cases $k \in\{2,3,4,5\}$. Cases $k \in\{2,3,4\}$ refer to the McCormick, Meyer-Floudas 


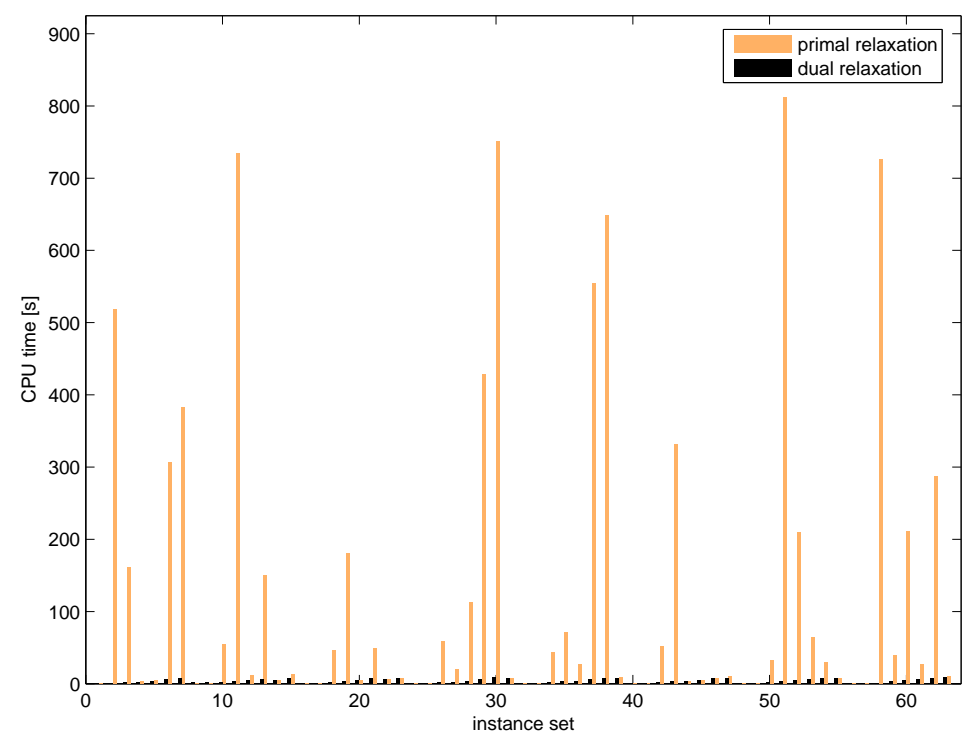

Figure 4.8: CPU time averages (in seconds) over each 20-instance set with given $(n, \beta, \tau)$ with $n=20$ for the MILP relaxations.

and Balram [18] inequalities. The statistic for $k=5$ is taken from [18], but devised computationally using a method similar to PORTA.

\begin{tabular}{rrr}
\hline$k$ & Primal & Dual \\
\hline 2 & 4 & 8 \\
3 & 12 & 13 \\
4 & 44 & 22 \\
5 & 130 & 39 \\
\hline
\end{tabular}

Table 4.1: Per-multilinear-term size increase (new constraints and variables) for primal and dual envelopes.

Numerical results confirm this behavior. Considering LP problems, although for $n=10$ (see Figure 4.5) the CPU time is very slightly in favor of the primal relaxation, the situation changes visibly for $n=20$ (see Figure 4.6). However, even if the CPU times differ, we cannot infer much on the comparative stability of the two methods.

Moving to the MILP problems, the CPU differences are decidedly striking in the case $n=10$ and even excessively so for the case $n=20$, as shown in Figures 4.7 and 4.8. The CPU time taken to solve primal relaxations is far from proportional to formulation size, whereas the stability associated to the dual relaxation is remarkable. 


\section{Part IV}

\section{Conclusions and bibliography}





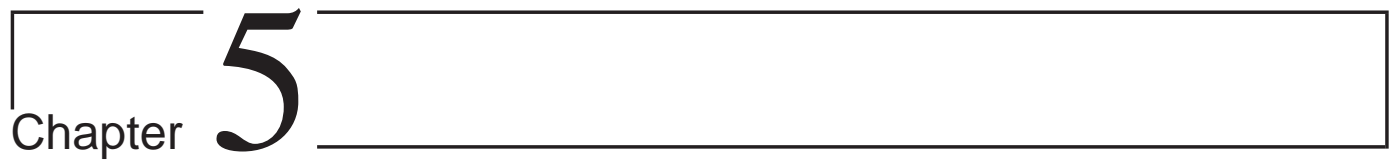

\section{Conclusions}

The main aim of this thesis is to convince the reader about the importance of reformulations in MP, both from a theoretical and a practical point of view. As a matter of fact, very often the most natural formulation to describe a problem is not the most efficient to be solved, and better results (for example in terms of computational time to get the optimal solution) can be achieved by reformulating the original model. It turns out that to perform this reformulation step in a profitable way one should know how the solvers work and what is the relationship (even approximately) between the kind of problem to solve (e.g., LP, MILP, NLP) and its complexity. This is very important to have somehow an "intuition" toward the best formulation, and to avoid reformulations that are harder to solve than the original formulation. For example, if one artificially reformulates a LP problem to a NLP problem, it is very likely that finding an optimal solution will take longer. This is the reason why in Chapter 1 we reported a short description of the different categories of MP problems (with some order relationships on their complexities) and solvers. However, there can be cases where it is not clear, before performing some numerical experiments, which, between the original formulation and a reformulation, is best. Consider for example the reformulation proposed in Chapter 3, where we replaced the constraints (2.22)-(2.23) with the constraints (2.34)-(2.35). It is not so obvious that the second set of inequalities is better than the first set, but some tests confirmed this fact. However, we tried to give an explanation of this behavior on the basis of our knowledge about the used solver.

In this thesis we followed the classification proposed by Liberti in [157]: exact reformulations, narrowings, and relaxations. Basically, each case corresponds to a chapter where we presented a problem and its MP formulation. Then we derived reformulations, motivated by a theoretical analysis of the problem. The order of presentation of the reformulations is related to a logical interpretation of the solution process of a difficult problem: starting from the original formulation, one can employ an exact reformulation, that does not alter the set of optimal solutions. If 
the problem is still difficult to solve, and it presents symmetries, narrowings reformulation can be used to make some optimal solutions infeasible with the guarantee that at least one is preserved. Finally, if the other techniques fail, one can relax the problem. This means that the solution of the corresponding relaxation does not provide the optimal solution of the original problem, but a lower (upper) bound in case of an objective function being minimized (maximized).

Chapter 2 deals with exact reformulations, and the problem studied is the clustering by means of modularity maximization. Actually in that chapter we also introduced another problem related to clustering, that can be described by means of MP, but due to the size of the resulting formulation we decided to implement a specific (as opposed to general-purpose) BB algorithm to solve it. However, concerning the modularity maximization problem, we considered a heuristic that solves a cMIQP problem at each step. We proposed some reformulations of this cMIQP formulation, that are basically compact models where we were able to decrease the number of variables and constraints by preserving the optimal solutions set. Moreover, the theoretical analysis underlined a symmetric structure of the problem, thus we also employed a SBC, yielding a narrowing (which is the main subject of Chapter 3). Thanks to these reformulations, the computational time to solve some classical instances by the heuristic was decreased by an order of magnitude. We also considered the extension of this method to bipartite graphs, and the MP model in this case in not a cMIQP but a MINLP, hence it is more difficult to solve. It is interesting to notice that the reformulation techniques yielding the best results for unipartite graphs are not the same as for bipartite graphs, even if the problems are strictly related. By the way, one of the reformulations used for the bipartite case (that is (2.101)-(2.108)) was really close to the original formulation for the general case, thus underlying the strict relationship between these problems. This was not clear by comparing the original formulations.

In Chapter 3 we studied the problem of packing equal circles in a square. As this problem involves a high degree of symmetries, it is a very good candidate for the application of narrowings. We were able to characterize the symmetric structure of the problem, and then to obtain some SBCs in an automatic way. Furthermore we derived some other SBCs that are more effective than the ones obtained automatically, in the sense that they lead to an improvement of the bounds provided by the sBB, giving good quality solutions already at the root of the sBB tree. We also proposed some other valid inequalities and finally we presented a conjecture about the reduction of the range for some variables of the problem. This last point is motivated by the fact that in the original formulation all the variables have the same range, and this causes a very poor relaxation of the problem (as computed at the root node of the sBB tree).

Finally, in Chapter 4 we compared two relaxations for problems involving multilinear terms. The first, called primal, defines the convex relaxation of the problem 
using a set of inequalites. The second one, called dual, represents the convex relaxation as the convex combination of the vertices of the convex hull using dual variables $\lambda$. The optimal solutions of these relaxations is the same for a given problem, thus we could say that one is an exact reformulation of the other. These relaxations are known in the literature, and usually the primal is the most used. We showed that for NLPs, and still more for MINLPs, the dual approach outperforms the primal in terms of computational time and size of the formulation.

It appears that the problems we studied are very different, but in every case we had some advantages by applying reformulation techniques. This is an indication of the fact that given a general problem, it is worth to spend some time to study it and to try to improve the original formulation.

The future work has two main directions. First, to perform an analysis of the different reformulation techniques which can be used fo a general problem. It is not easy, as the efficacy of the reformulations is very often related to the specific problem. However, we have some examples in this thesis, as the constraints (2.34)(2.35) which in case of maximization problems can be used in place of (2.22)-(2.23), or the automatic symmetry detection techniques presented in Chapter 3. Second, once these reformulations techniques are formalized, to integrate them in a solver. Indeed this is a hard task, because the human analysis of the problem can detect some particular features of a problem that are difficult to find automatically, but its importance is crucial, above all in Operations Research where very often a user models a problem having only a limited knowledge about the solution process and without trying to improve the first formulation obtained. 


\section{Bibliography}

[1] K. Abhishek, S. Leyffer, and J. Linderoth. FilMINT: An Outer ApproximationBased Solver for Convex Mixed-Integer Nonlinear Programs. INFORMS Journal on Computing, 22(4):555-567, 2010.

[2] W. P. Adams and P. M. Dearing. On the equivalence between roof duality and Lagrangian duality for unconstrained $0-1$ quadratic programming problems. Discrete Applied Mathematics, 48(1):1-20, 1994.

[3] B. Addis, M. Locatelli, and F. Schoen. Disk Packing in a Square: A New Global Optimization Approach. INFORMS Journal on Computing, 20(4):516524, 2008.

[4] N. Adhya, M. Tawarmalani, and N. V. Sahinidis. A Lagrangian Approach to the Pooling Problem. Industrial \& Engineering Chemistry Research, 38(5):1956-1972, 1999.

[5] C. S. Adjiman, S. Dallwig, C. A. Floudas, and A. Neumaier. A global optimization method, $\alpha \mathrm{BB}$, for general twice-differentiable constrained NLPs: I. Theoretical advances. Computers \& Chemical Engineering, 22(9):1137-1158, 1998 .

[6] G. Adomavicius and A. Tuzhilin. Toward the next generation of recommender systems: A survey of the state-of-the-art and possible extensions. IEEE Transactions on Knowledge and Data Engineering, 17(6):734-749, 2005.

[7] A. Agarwal, S. Bhat, A. Gray, and I. E. Grossmann. Automating Mathematical Program Transformations. In M. Carro and R. Peña, editors, Proceedings of the $12^{\text {th }}$ International Symposium on Practical Aspects of Declarative Languages (PADL), volume 5937 of Lecture Notes in Computer Science, pages 134-148. Springer Berlin / Heidelberg, 2010.

[8] G. Agarwal and D. Kempe. Modularity-Maximizing Graph Communities via Mathematical Programming. The European Physical Journal B, 66(3):409418, 2008. 
[9] F. Al-Khayyal and J. Falk. Jointly Constrained Biconvex Programming. Mathematics of Operations Research, 8(2):273-286, 1983.

[10] D. Aloise, S. Cafieri, G. Caporossi, P. Hansen, S. Perron, and L. Liberti. Column generation algorithms for exact modularity maximization in networks. Physical Review E, 82(4):046112, 2010.

[11] K. Anstreicher. Semidefinite programming versus the reformulationlinearization technique for nonconvex quadratically constrained quadratic programming. Journal of Global Optimization, 43(2):471-484, 2009.

[12] D. L. Applegate, R. E. Bixby, V. Chvátal, and W. J. Cook. The Traveling Salesman: a Computational Study. Princeton University Press, Princeton, 2007.

[13] A. Arenas, A. Fernández, and S. Gómez. Analysis of the structure of complex networks at different resolution levels. New Journal of Physics, 10(5):053039, 2008 .

[14] C. Audet, J. Brimberg, P. Hansen, S. Le Digabel, and N. Mladenović. Pooling Problem: Alternate Formulations and Solution Methods. Management Science, 50(6):761-776, 2004.

[15] C. Audet, P. Hansen, B. Jaumard, and G. Savard. Links between linear bilevel and mixed $0-1$ programming problems. Journal of Optimization Theory and Applications, 93(2):273-300, 1997.

[16] M. Avriel and B. Golany. Mathematical Programming for Industrial Engineers. Marcel Dekker, 1996.

[17] B. Ball, B. Karrer, and M. E. J. Newman. Efficient and principled method for detecting communities in networks. Physical Review E, 84(3):036103, 2011.

[18] S. Balram. Crude transshipment via floating, production, storage and offloading platforms. Master's thesis, Deptartment of Chemical and Biomolecular Engineering, National University of Singapore, 2010.

[19] M. J. Barber. Modularity and community detection in bipartite networks. Physical Review E, 76(6):066102, 2007.

[20] M. J. Barber and J. W. Clark. Detecting network communities by propagating labels under constraints. Physical Review E, 80(2):026129, 2009.

[21] C. Barnhart and G. Laporte, editors. Transportation, volume 14 of Handbooks in Operations Research and Management Science. North-Holland, Amsterdam, 2007. 
[22] V. Batagelj and A. Mrvar. Pajek datasets, 2006.

[23] S. Bazaraa, H. D. Sherali, and C. M. Shetty. Nonlinear Programming: Theory and Algorithms. Wiley-Interscience, 2006.

[24] P. Belotti, S. Cafieri, J. Lee, and L. Liberti. Feasibility-based bounds tightening via fixed points. In D.-Z. Du, P. M. Pardalos, and B. Thuraisingham, editors, Proceedings of the $4^{\text {th }}$ International Conference on Combinatorial Optimization and Applications (COCOA), volume 6508 of Lecture Notes in Computer Science, pages 65-76. Springer, New York, 2010.

[25] P. Belotti, S. Cafieri, J. Lee, L. Liberti, and A. Miller. On the composition of convex envelopes for quadrilinear terms. In P. M. Pardalos, editor, Optimization and Optimal Control, Nonconvex Optimization and Its Application. Springer, New York, to appear.

[26] P. Belotti, J. Lee, L. Liberti, F. Margot, and A. Wächter. Branching and bounds tightening techniques for non-convex MINLP. Optimization Methods and Software, 24(4-5):597-634, 2009.

[27] A. Ben-Israel and B. Mond. What is invexity? Journal of Australian Mathematical Society B, 28(1):1-9, 1986.

[28] L. Bertacco, M. Fischetti, and A. Lodi. A feasibility pump heuristic for general mixed-integer problems. Discrete Optimization, 4(1):63-76, 2007.

[29] T. Berthold and A. Gleixner. Undercover - Primal MINLP Heuristic. In P. Bonami, L. Liberti, A. Miller, and A. Sartenaer, editors, Proceedings of the European Workshop on Mixed-Integer Nonlinear Programming (EWMINLP), pages 103-113. Université de la Méditerranée, Marseille, 2010.

[30] R. E. Bixby. Solving Real-World Linear Programs: A Decade and More of Progress. Operations Research, 50(1):3-15, 2002.

[31] S. Boccaletti, M. Ivanchenko, V. Latora, A. Pluchino, and A. Rapisarda. Detecting complex network modularity by dynamical clustering. Physical Review E, 75(4):045102, 2007.

[32] D. W. Boll, J. Donovan, R. L. Graham, and B. D. Lubachevsky. Improving Dense Packings of Equal Disks in a Square. Electronic Journal of Combinatorics, 7(1, R46):1-9, 2000.

[33] M. Bolla. Penalized versions of the Newman-Girvan modularity and their relation to normalized cuts and $k$-means clustering. Physical Review E, 84(1):016108, 2011. 
[34] P. Bonami, G. Cornuéjols, A. Lodi, and F. Margot. A Feasibility Pump for mixed integer nonlinear programs. Mathematical Programming, 119(2):331$352,2009$.

[35] P. Bonami and J. P. M. Gonçalves. Primal heuristics for mixed integer nonlinear programs. Technical Report RC24639, IBM, 2008.

[36] P. Bonami and J. Lee. Bonmin user's manual. Technical report, IBM, June 2007.

[37] M. Boulle. Compact Mathematical Formulation for Graph Partitioning. Optimization and Engineering, 5(3):315-333, 2004.

[38] S. Bradley, A. Hax, and T. Magnanti. Applied Mathematical Programming. Addison Wesley, 1977.

[39] U. Brandes, D. Delling, M. Gaertler, R. Görke, M. Hoefer, Z. Nikoloski, and D. Wagner. On Modularity Clustering. IEEE Transactions on Knowledge and Data Engineering, 20(2):172-188, 2008.

[40] H. Breu and D. Kirkpatrick. On the complexity of recognizing intersection and touching graphs of disks. In F. Brandenburg, editor, Graph Drawing, volume 1027 of Lecture Notes in Computer Science, pages 88-98. Springer, Berlin, 1996.

[41] J. Brimberg, P. Hansen, N. Mladenović, and E. Taillard. Improvement and Comparison of Heuristics for Solving the Uncapacitated Multisource Weber Problem. Operations Research, 48(3):444-460, 2000.

[42] A. Brook, D. Kendrick, and A. Meeraus. GAMS, a user's guide. ACM SIGNUM Newsletter, 23(3-4):10-11, 1988.

[43] G. G. Brown and R. F. Dell. Formulating integer linear programs: A rogues' gallery. INFORMS Transactions on Education, 7:1-13, 2007.

[44] S. Cafieri, G. Caporossi, P. Hansen, S. Perron, and A. Costa. Finding communities in networks in the strong and almost-strong sense. Physical Review E, 85(4):046113, 2012.

[45] S. Cafieri, A. Costa, and P. Hansen. Reformulation of a model for hierarchical divisive graph modularity maximization. Annals of Operations Research, in revision.

[46] S. Cafieri, P. Hansen, and L. Liberti. Loops and multiple edges in modularity maximization of networks. Physical Review E, 81(4):046102, 2010.

[47] S. Cafieri, P. Hansen, and L. Liberti. Locally optimal heuristic for modularity maximization of networks. Physical Review E, 83(5):056105, 2011. 
[48] S. Cafieri, P. Hansen, and L. Liberti. Improving heuristics for network modularity maximization using an exact algorithm. Discrete Applied Mathematics, to appear.

[49] S. Cafieri, J. Lee, and L. Liberti. On convex relaxations of quadrilinear terms. Journal of Global Optimization, 47(4):661-685, 2010.

[50] G. Caporossi and P. Hansen. Variable neighborhood search for extremal graphs: 1 The AutoGraphiX system. Discrete Mathematics, 212(1-2):29-44, 2000 .

[51] L. Casado, I. García, P. Szabó, and T. Csendes. Equal circles packing in square II: new results for up to 100 circles using the TAMSASS-PECS algorithm. In F. Giannessi, P. M. Pardalos, and T. Rapcsak, editors, Optimization Theory: Recent Developments from Mátraháza, pages 207-224. Kluwer, Dordrecht, 2001.

[52] I. Castillo, F. Kampas, and J. Pintér. Solving circle packing problems by global optimization: Numerical results and industrial applications. European Journal of Operational Research, 191(3):786-802, 2008.

[53] B. Chachuat. Nonlinear and Dynamic Optimization: From Theory to Practice. Automatic Control Laboratory, EPFL, Switzerland, 2007.

[54] P. K. Chan, M. D. F. Schlag, and J. Y. Zien. Spectral K-way ratio-cut partitioning and clustering. IEEE Transactions on CAD of Integrated Circuits and Systems, 13(9):1088-1096, 1994.

[55] T. Christof and A. Löbel. The porta manual page. Technical Report v. 1.4.0, ZIB, Berlin, 1997.

[56] V. Chvátal. Linear Programming. Series of Books in the Mathematical Sciences. W. H. Freeman, 1983.

[57] A. Clauset, M. E. J. Newman, and C. Moore. Finding community structure in very large networks. Physical Review E, 70(6):066111, 2004.

[58] A. Costa and P. Hansen. Comment on "Evolutionary method for finding communities in bipartite networks". Physical Review E, 84(5):058101, 2011.

[59] A. Costa, P. Hansen, and L. Liberti. Static symmetry breaking in circle packing. In U. Faigle, R. Schrader, and D. Herrmann, editors, Proceedings of the $9^{\text {th }}$ Cologne-Twente Workshop on Graphs and Combinatorial Optimization (CTW), pages 47-50. University of Köln, 2010.

[60] A. Costa, P. Hansen, and L. Liberti. Bound constraints for Point Packing in a Square. In L. Adacher, M. Flamini, G. Leo, G. Nicosia, A. Pacifici, 
and V. Piccialli, editors, Proceedings of the $10^{\text {th }}$ Cologne-Twente Workshop on Graphs and Combinatorial Optimization (CTW), pages 126-129. Università di Roma "Tor Vergata", Villa Mondragone, 2011.

[61] A. Costa, P. Hansen, and L. Liberti. On the impact of symmetry-breaking constraints on spatial Branch-and-Bound for circle packing in a square. Discrete Applied Mathematics, 161(1-2):96-106, 2013.

[62] A. Costa and L. Liberti. Relaxations of Multilinear Convex Envelopes: Dual Is Better Than Primal. In R. Klasing, editor, Proceedings of the $11^{\text {th }}$ International Symposium on Experimental Algorithms (SEA), volume 7276 of Lecture Notes in Computer Science, pages 87-98. Springer, 2012.

[63] A. Costa, L. Liberti, and P. Hansen. Formulation symmetries in circle packing. In A. R. Mahjoub, editor, Proceedings of the $1^{\text {st }}$ International Symposium on Combinatorial Optimization (ISCO), volume 36 of Electronic Notes in Discrete Mathematics, pages 1303-1310. Elsevier, 2010.

[64] A. Costa and I. Tseveendorj. Symmetry breaking constraints for the problem of packing equal circles in a square. In C. J. Luz and F. Valente, editors, Proceedings of the $1^{\text {st }}$ International Conference on Operations Research and Enterprise Systems (ICORES), pages 5-10. SciTePress, 2012.

[65] B. D. Craven and B. M. Glover. Invex functions and duality. Journal of the Australian Mathematical Society A, 39(1):1-20, 1985.

[66] Y. Cui. Generating optimal t-shape cutting patterns for circular blanks. Computers $\& 3$ Operations Research, 32(1):143-152, 2005.

[67] C. D'Ambrosio, J. Linderoth, and J. Luedtke. Valid Inequalities for the Pooling Problem with Binary Variables. In O. Günlük and G. Woeginger, editors, Proceedings of the $15^{\text {th }}$ Integer Programming and Combinatorial Optimization Conference (IPCO), volume 6655 of Lecture Notes in Computer Science, pages 117-129. Springer / Heidelberg, 2011.

[68] E. Danna, E. Rothberg, and C. Le Pape. Exploring relaxation induced neighborhoods to improve MIP solutions. Mathematical Programming, 102(1):7190, 2005.

[69] L. Danon, A. Díaz-Guilera, and A. Arenas. The effect of size heterogeneity on community identification in complex networks. Journal of Statistical Mechanics: Theory and Experiment, 2006(11):P11010, 2006.

[70] G. B. Dantzig. Linear Programming and Extensions. Princeton University Press, Princeton, 1963. 
[71] L. Dartnell, E. Simeonidis, M. Hubank, S. Tsoka, I. D. L. Bogle, and L. G. Papageorgiou. Robustness of the p53 network and biological hackers. FEBS letters, 579(14):3037-3042, 2005.

[72] T. Davidović, L. Liberti, N. Maculan, and N. Mladenović. Towards the optimal solution of the multiprocessor scheduling problem with communication delays. In P. Baptiste, G. Kendall, A. Munier-Kordon, and F. Sourd, editors, Proceedings of the $3^{\text {rd }}$ Multidisciplinary International Conference on Scheduling: Theory and Applications (MISTA), pages 128-135, Paris, 2007.

[73] A. Davis, B. B. Gardner, and M. R. Gardner. Deep South: A Social Anthropological Study of Caste and Class. University of Chicago Press, Chicago, 1941.

[74] P. J. Davis. Packing inequalities for circles. Michigan Mathematical Journal, 10(1):25-31, 1963.

[75] E. Demaine, S. Fekete, and R. Lang. Circle packing for origami design is hard. In A. K. Peters, editor, Proceedings of the 5th International Conference on Origami in Science, Mathematics and Education (OSME), pages 609-626, Singapore, 2010.

[76] T. N. Dinh and M. T. Thai. Finding Community Structure with Performance Guarantees in Complex Networks. Technical report, arXiv:1108.4034, 2011.

[77] H. N. Djidjev. A Scalable Multilevel Algorithm for Graph Clustering and Community Structure Detection. In W. Aiello, A. Broder, J. Janssen, and E. Milios, editors, Proceedings of the $4^{\text {th }}$ Workshop on Algorithms and Models for the Web-Graph (WAW), volume 4936 of Lecture Notes in Computer Science, pages 117-128. Springer Berlin / Heidelberg, 2008.

[78] C. O. Dorso and A. D. Medus. Community Detection in Networks. International Journal of Bifurcation and Chaos, 20(2):361-367, 2010.

[79] O. du Merle, P. Hansen, B. Jaumard, and N. Mladenović. An Interior Point Algorithm for Minimum Sum-of-Squares Clustering. SIAM Journal on Scientific Computing, 21(4):1485-1505, 2000.

[80] J. Duch and A. Arenas. Community detection in complex networks using extremal optimization. Physical Review E, 72(2):027104, 2005.

[81] M. A. Duran and I. E. Grossmann. An outer-approximation algorithm for a class of mixed-integer nonlinear programs. Mathematical Programming, 36(3):307-339, 1986.

[82] P. Eades and N. Wormald. Fixed edge-length graph drawing is NP-hard. Discrete Applied Mathematics, 28(2):111-134, 1990. 
[83] J. E. Falk and R. M. Soland. An Algorithm for Separable Nonconvex Programming Problems. Management Science, 15(9):550-569, 1969.

[84] N. Fan and P. M. Pardalos. Linear and quadratic programming approaches for the general graph partitioning problem. Journal of Global Optimization, 48(1):57-71, 2010.

[85] R. R. Faulkner. Music on Demand: Composers and Careers in the Hollywood Film Industry. Transaction Publishers, 2003.

[86] M. C. Ferris, J. Lim, and D. M. Shepard. An Optimization Approach for Radiosurgery Treatment Planning. SIAM Journal on Optimization, 13(3):921937, 2002.

[87] M. Fischetti. Lezioni di Ricerca Operativa. Edizioni Libreria Progetto Padova, second edition, 1999.

[88] M. Fischetti, F. Glover, and A. Lodi. The feasibility pump. Mathematical Programming, 104(1):91-104, 2005.

[89] M. Fischetti and A. Lodi. Local branching. Mathematical Programming, 98(13):23-37, 2005.

[90] G. W. Flake, S. Lawrence, C. L. Giles, and F. M. Coetzee. Self-Organization and Identification of Web Communities. IEEE Computer, 35(3):66-71, 2002.

[91] C. A. Floudas. Deterministic Global Optimization: Theory, Methods and Applications. Kluwer Academic Publishers, Dordrecht, 2000.

[92] C. A. Floudas and P. M. Pardalos, editors. Encyclopedia of Optimization. Springer, New York, second edition, 2009.

[93] R. Fortet. Applications de l'algèbre de Boole en recherche opérationelle. Revue Française d'Informatique et de Recherche Opérationelle, 4(14):17-26, 1960.

[94] S. Fortunato. Community detection in graphs. Physics Reports, 486(3-5):75$174,2010$.

[95] S. Fortunato and M. Barthélemy. Resolution limit in community detection. Proceedings of the National Academy of Sciences of the U.S.A., 104(1):36-41, 2007.

[96] L. R. Foulds, D. Haughland, and K. Jörnsten. A Bilinear Approach to the Pooling Problem. Optimization, 24(1-2):165-180, 1992.

[97] R. Fourer and D. Gay. The AMPL Book. Duxbury Press, Pacific Grove, 2002. 
[98] R. J. Fowler, M. Paterson, and S. Tanimoto. Optimal packing and covering in the plane are NP-complete. Information Processing Letters, 12(3):133-137, 1981.

[99] H. J. Fraser and J. A. George. Integrated container loading software for pulp and paper industry. European Journal of Operational Research, 77(3):466-474, 1994.

[100] K. Fukuda and A. Prodon. Double description method revisited. In M. Deza, R. Euler, and Y. Manoussakis, editors, proceedings of the 8th Franco-Japanese and 4th Franco-Chinese Conference on Combinatorics and Computer Science (CCS), volume 1120 of Lecture Notes in Computer Science, pages 91-111. Springer, London, 1995.

[101] M. R. Garey and D. S. Johnson. Computers and Intractability: A Guide to the Theory of NP-Completeness. Freeman and Company, New York, 1979.

[102] A. M. Geoffrion. Generalized Benders decomposition. Journal of Optimization Theory and Applications, 10(4):237-260, 1972.

[103] J. A. George, J. M. George, and B. W. Lamar. Packing different-sized circles into a rectangular container. European Journal of Operational Research, 84(3):693-712, 1995.

[104] P. Gill. User's guide for SNOPT version 7. Systems Optimization Laboratory, Stanford University, California, 2006.

[105] M. Girvan and M. E. J. Newman. Community structure in social and biological networks. Proceedings of the National Academy of Sciences of the U.S.A., 99(12):7821-7826, 2002.

[106] F. Glover and G. Kochenberger, editors. Handbook of Metaheuristics. Kluwer, Dordrecht, 2003.

[107] R. E. Gomory. Outline of an algorithm for integer solutions to linear programs. Bulletin of the American Mathematical Society, 64(5):275-278, 1958.

[108] B. H. Good, Y.-A. de Montjoye, and A. Clauset. Performance of modularity maximization in practical contexts. Physical Review E, 81(4):046106, 2010.

[109] E. Goubault, S. Le Roux, J. Leconte, L. Liberti, and F. Marinelli. Static analysis by abstract interpretation: a mathematical programming approach. In A. Miné and E. Rodríguez-Carbonell, editors, Proceeding of the $2^{\text {nd }}$ International Workshop on Numerical and Symbolic Abstract Domains (NSAD), volume 267(1) of Electronic Notes in Theoretical Computer Science, pages 7387. Elsevier, 2010. 
[110] R. L. Graham and B. D. Lubachevsky. Repeated Patterns of Dense Packings of Equal Disks in a Square. Electronic Journal of Combinatorics, 3(1, R16):1-17, 1996.

[111] I. E. Grossmann, J. A. Caballero, and H. Yeomans. Mathematical programming approaches to the synthesis of chemical process systems. Korean Journal of Chemical Engineering, 16(4):407-426, 1999.

[112] A. Grosso, A. R. M. J. U. Jamali, M. Locatelli, and F. Schoen. Solving the problem of packing equal and unequal circles in a circular container. Journal of Global Optimization, 47(1):63-81, 2010.

[113] A. Grosso, M. Locatelli, and F. Schoen. Solving molecular distance geometry problems by global optimization algorithms. Computational Optimization and Applications, 43(1):23-27, 2009.

[114] M. Grötschel and Y. Wakabayashi. A cutting plane algorithm for a clustering problem. Mathematical Programming, 45(1-3):59-96, 1989.

[115] R. Guimerà and L. A. N. Amaral. Functional cartography of complex metabolic networks. Nature, 433(7028):895-900, 2005.

[116] O. K. Gupta and A. Ravindran. Branch and Bound Experiments in Convex Nonlinear Integer Programming. Management Science, 31(12):1533-1546, 1985.

[117] Gurobi Optimization Inc. Gurobi Optimizer Reference Manual, 2012.

[118] L. Hagen and A. B. Kahng. New spectral methods for ratio cut partitioning and clustering. IEEE Transactions on CAD of Integrated Circuits and Systems, 11(9):1074-1085, 1992.

[119] P. Hammer and S. Rudeanu. Boolean Methods in Operations Research and Related Areas. Springer, Berlin, 1968.

[120] P. Hansen, J. Brimberg, N. Mladenović, and D. Urosević. Primal-dual variable neighborhood search for the simple plant-location problem. INFORMS Journal on Computing, 19(4):552-564, 2007.

[121] P. Hansen and B. Jaumard. Cluster analysis and mathematical programming. Mathematical Programming, 79(1-3):191-215, 1997.

[122] M. A. Hanson. On sufficiency of the Kuhn-Tucker conditions. Journal of Mathematical Analysis and Applications, 80(2):545-550, 1981.

[123] M. A. Hanson. Invexity and the Kuhn-Tucker Theorem. Journal of Mathematical Analysis and Applications, 236(2):594-604, 1999. 
[124] C. A. Haverly. Studies of the behaviour of recursion for the pooling problem. ACM SIGMAP Bulletin, 25:19-28, 1978.

[125] J. Herskovits, P. Mappa, E. Goulart, and C. M. Mota Soares. Mathematical programming models and algorithms for engineering design optimization. Computer Methods in Applied Mechanics and Engineering, 194(30-33):3244$3268,2005$.

[126] M. Hifi and R. M'Hallah. Approximate algorithms for constrained circular cutting problems. Computers \& Operations Research, 31(5):675-694, 2004.

[127] M. Hifi and R. M'Hallah. A Literature Review on Circle and Sphere Packing Problems: Models and Methodologies. Advances in Operations Research, 2009:150624, 2009.

[128] M. Hifi, V. Th. Paschos, and V. Zissimopoulos. A simulated annealing approach for the circular cutting problem. European Journal of Operational Research, 159(2):430-448, 2004.

[129] D. S. Hochbaum and W. Maass. Approximation schemes for covering and packing problems in image processing and VLSI. Journal of the ACM, 32(1):130136, 1985.

[130] E. Hopper and B. Turton. Application of genetic algorithms to packing problems - a review. In P. K. Chawdry, R. Roy, and R. K. Kant, editors, Proceedings of the $2^{\text {nd }}$ On-line World Conference of Soft Computing in Engineering Design and Manufacturing, pages 279-288. Springer, Berlin, 1997.

[131] R. Horst and N. V. Thoai. Dc programming: Overview. Journal of Optimization Theory and Applications, 103(1):1-43, 1999.

[132] Y. Hu, H. Chen, P. Zhang, M. Li, Z. Di, and Y. Fan. Comparative definition of community and corresponding identifying algorithm. Physical Review E, 78(2):026121, 2008.

[133] W. Huang and T. Ye. Greedy vacancy search algorithm for packing equal circles in a square. Operations Research Letters, 38(5):378-382, 2010.

[134] V. Hugo. Les Misérables. Gallimard, Bibliothèque de la Pléiade, Paris, 1951.

[135] IBM. ILOG CPLEX 12.2 User's Manual. IBM, 2010.

[136] W. Imrich and S. Klavžar. Products Graphs, Structure and Recognition. John Wiley and Sons, New York, 2000.

[137] B. Jaumard, P. Hansen, and M. P. de Aragão. Column generation methods for probabilistic logic. In R. Kannan and W. R. Pulleyblank, editors, Proceedings 
of the $1^{\text {st }}$ Integer Programming and Combinatorial Optimization Conference (IPCO), pages 313-331. University of Waterloo Press, 1990.

[138] D. S. Johnson. The NP-completeness column: An ongoing guide. Journal of Algorithms, 3(2):182-195, 1982.

[139] N. Karmarkar. A new polynomial time algorithm for linear programming. Combinatorica, 4(4):373-395, 1984.

[140] W. Karush. Minima of Functions of Several Variables with Inequalities as Side Constraints. Master's thesis, Department of Mathematics, University of Chicago, 1939.

[141] D. E. Knuth. The Art of Computer Programming, Part II: Seminumerical Algorithms. Addison-Wesley, Reading, MA, 1981.

[142] D. E. Knuth. The Stanford GraphBase: a platform for combinatorial computing. Addison-Wesley, 1993.

[143] S. Kucherenko, P. Belotti, L. Liberti, and N. Maculan. New formulations for the kissing number problem. Discrete Applied Mathematics, 155(14):18371841, 2007.

[144] H. W. Kuhn and A. W. Tucker. Nonlinear Programming. In J. Neyman, editor, Proceedings of the 2nd Berkeley Symposium on Mathematical Statistics and Probability, pages 481-492. University of California Press, Berkeley, CA, USA, 1950.

[145] J. M. Kumpula, J. Saramäki, K. Kaski, and J. Kertész. Limited resolution and multiresolution methods in complex network community detection. Fluctuations and Noise Letters, 7(3):209-214, 2007.

[146] M. Labbé, D. Peeters, and J.-F. Thisse. Location on networks. In M. Ball, T. Magnanti, C. Monma, and G. Nemhauser, editors, Network Routing, volume 8 of Handbooks in Operations Research and Management Science. NorthHolland, Amsterdam, 1995.

[147] G. Lancia. Mathematical Programming in Computational Biology: an Annotated Bibliography. Algorithms, 1(2):100-129, 2008.

[148] A. H. Land and A. G. Doig. An Automatic Method of Solving Discrete Programming Problems. Econometrica, 28(3):497-520, 1960.

[149] C. Lavor, L. Liberti, N. Maculan, and M. A. C. Nascimento. Solving Hartree-Fock systems with global optimization methods. Europhysics Letters, 77(5):50006, 2007. 
[150] C. Lavor, L. Liberti, A. Mucherino, and N. Maculan. On a discretizable subclass of instances of the molecular distance geometry problem. In D. Shin, editor, Proceedings of the 24th Annual ACM Symposium on Applied Computing (SAC), pages 804-805. ACM, 2009.

[151] E. A. Leicht and M. E. J. Newman. Community Structure in Directed Networks. Physical Review Letters, 100(11):118703, 2008.

[152] Z. Li, S. Zhang, R.-S. Wang, X.-S. Zhang, and L. Chen. Quantitative function for community detection. Physical Review E, 77(3):036109, 2008.

[153] L. Liberti. Reformulation and Convex Relaxation Techniques for Global Optimization. PhD thesis, Imperial College London, UK, 2004.

[154] L. Liberti. Writing Global Optimization Software. In L. Liberti and N. Maculan, editors, Global Optimization: from Theory to Implementation, volume 84, pages 211-262. Springer, Berlin, 2006.

[155] L. Liberti. Automatic Generation of Symmetry-Breaking Constraints. In B. Yang, D.-Z. Du, and C. Wang, editors, Proceedings of the $2^{\text {nd }}$ Annual International Conference on Combinatorial Optimization and Applications (COCOA), volume 5165 of Lecture Notes in Computer Science, pages 328-338. Springer, Berlin, 2008.

[156] L. Liberti. Introduction to Global Optimization. Technical report, LIX, École Polytechnique, 2008.

[157] L. Liberti. Reformulations in Mathematical Programming: Definitions and Systematics. RAIRO-OR, 43(1):55-86, 2009.

[158] L. Liberti. Reformulations in mathematical programming: automatic symmetry detection and exploitation. Mathematical Programming, 131(1-2):273-304, 2012 .

[159] L. Liberti. Symmetry in Mathematical Programming. In J. Lee and S. Leyffer, editors, Mixed Integer Nonlinear Programming, volume 154 of The IMA Volumes in Mathematics and its Application, pages 263-286. Springer, New York, 2012.

[160] L. Liberti, S. Cafieri, and F. Tarissan. Reformulations in Mathematical Programming: A Computational Approach. In A. Abraham, A.-E. Hassanien, P. Siarry, and A. Engelbrecht, editors, Foundations of Computational Intelligence Volume 3, number 203 in Studies in Computational Intelligence, pages 153-234. Springer, Berlin, 2009. 
[161] L. Liberti, C. Lavor, and N. Maculan. A Branch-and-Prune algorithm for the Molecular Distance Geometry Problem. International Transactions in Operational Research, 15(1):1-17, 2008.

[162] L. Liberti, C. Lavor, N. Maculan, and F. Marinelli. Double variable neighbourhood search with smoothing for the molecular distance geometry problem. Journal of Global Optimization, 43(2-3):207-218, 2009.

[163] L. Liberti, C. Lavor, N. Maculan, and M. A. C. Nascimento. Reformulation in mathematical programming: An application to quantum chemistry. Discrete Applied Mathematics, 157(6):1309-1318, 2009.

[164] L. Liberti, C. Lavor, A. Mucherino, and N. Maculan. Molecular distance geometry methods: from continuous to discrete. International Transactions in Operational Research, 18(1):33-51, 2010.

[165] L. Liberti, S. Le Roux, J. Leconte, and F. Marinelli. Mathematical programming based debugging. In A. R. Mahjoub, editor, Proceedings of the $1^{\text {st }}$ International Symposium on Combinatorial Optimization (ISCO), volume 36 of Electronic Notes in Discrete Mathematics, pages 1311-1318. Elsevier, 2010.

[166] L. Liberti, N. Maculan, and Y. Zhang. Optimal configuration of gamma ray machine radiosurgery units: the sphere covering subproblem. Optimization Letters, 3(1):109-121, 2009.

[167] L. Liberti and C. C. Pantelides. An Exact Reformulation Algorithm for Large Nonconvex NLPs Involving Bilinear Terms. Journal of Global Optimization, 36(2):161-189, 2006.

[168] G. J. Lim, M. C. Ferris, S. J. Wright, D. M. Shepard, and M. A. Earl. An Optimization Framework for Conformal Radiation Treatment Planning. INFORMS Journal on Computing, 19(3):366-380, 2007.

[169] X. Liu and T. Murata. Community Detection in Large-Scale Bipartite Networks. In Proceedings of the IEEE/WIC/ACM International Conference on Web Intelligence and Intelligent Agent Technologies, pages 50-57. IEEE, 2009.

[170] X. Liu and T. Murata. Advanced modularity-specialized label propagation algorithm for detecting communities in networks. Physica A, 389(7):1493$1500,2010$.

[171] X. Liu and T. Murata. An Efficient Algorithm for Optimizing Bipartite Modularity in Bipartite Networks. Journal of Advanced Computational Intelligence and Intelligent Informatics, 14(4):408-415, 2010. 
[172] M. Locatelli and U. Raber. Packing Equal Circles in a Square: I. Theoretical Results. Technical Report 08-99, Dipartimento Sistemi e Informatica, Università di Firenze, 1999.

[173] M. Locatelli and U. Raber. Packing equal circles in a square: a deterministic global optimization approach. Discrete Applied Mathematics, 122(1-3):139$166,2002$.

[174] F. Luccio and M. Sami. On the Decomposition of Networks in Minimally Interconnected Subnetworks. IEEE Transactions on Circuit Theory, 16(2):184-188, 1969.

[175] D. Lusseau, K. Schneider, O. J. Boisseau, P. Haase, E. Slooten, and S. M. Dawson. The bottlenose dolphin community of Doubtful Sound features a large proportion of long-lasting associations. Behavioral Ecology and Sociobiology, 54(4):396-405, 2003.

[176] O. L. Mangasarian, W. N. Street, and W. H. Wolberg. Breast Cancer Diagnosis and Prognosis Via Linear Programming. Operations Research, 43(4):570-577, 1995.

[177] C. D. Maranas and C. A. Floudas. Global Optimization in Generalized Geometric Programming. Computers \& Chemical Engineering, 21(4):351-369, 1997.

[178] C. D. Maranas, C. A. Floudas, and P. M. Pardalos. New results in the packing of equal circles in a square. Discrete Mathematics, 142(1-3):287-293, 1995.

[179] F. Margot. Symmetry in Integer Linear Programming. In M. Jünger, T. M. Liebling, D. Naddef, G. L. Nemhauser, W. R. Pulleyblank, G. Reinelt, G. Rinaldi, and L. A. Wolsey, editors, 50 Years of Integer Programming, pages 647-681. Springer, Berlin, 2010.

[180] D. H. Martin. The essence of invexity. Journal of Optimization Theory and Applications, 47(1):65-76, 1985.

[181] Y. Masmoudi, H. Chabchoub, S. Hanafi, and A. Rebaï. A Mathematical Programming Based Procedure for Breast Cancer Classification. Journal of Mathematical Modelling and Algorithms, 9(3):247-255, 2010.

[182] C. P. Massen and J. P. K. Doye. Identifying communities within energy landscapes. Physical Review E, 71(4):046101, 2005.

[183] G. McCormick. Computability of global solutions to factorable nonconvex programs: Part I - Convex underestimating problems. Mathematical Programming, 10(1):146-175, 1976. 
[184] A. D. Medus, G. Acuña, and C. O. Dorso. Detection of community structures in networks via global optimization. Physica A, 358(2-4):593-604, 2005.

[185] A. D. Medus and C. O. Dorso. Alternative approach to community detection in networks. Physical Review E, 79(6):066111, 2009.

[186] J. Mei, S. He, G. Shi, Z. Wang, and W. Li. Revealing network communities through modularity maximization by a contraction-dilation method. New Journal of Physics, 11(4):043025, 2009.

[187] C. A. Meyer and C. A. Floudas. Trilinear Monomials with Positive or Negative Domains: Facets of the Convex and Concave Envelopes. In C. A. Floudas and P. M. Pardalos, editors, Frontiers in Global Optimization, pages 327-352. Kluwer Academic Publishers, Amsterdam, 2003.

[188] C. A. Meyer and C. A. Floudas. Trilinear Monomials with Mixed Sign Domains: Facets of the Convex and Concave Envelopes. Journal of Global Optimization, 29(2):125-155, 2004.

[189] J. H. Michael. Labor dispute reconciliation in a forest products manufacturing facility. Forest Products Journal, 47(11-12):41-45, 1997.

[190] R. Milo, S. Itzkovitz, N. Kashtan, R. Levitt, S. Shen-Orr, I. Ayzenshtat, M. Sheffer, and U. Alon. Superfamilies of Evolved and Designed Networks. Science, 303(5663):1538-1542, 2004.

[191] R. Misener and C. A. Floudas. Global optimization of large-scale generalized pooling problems: Quadratically constrained minlp models. Industrial Engineering \& Chemical Research, 49(11):5424-5438, 2010.

[192] A. Mucherino and C. Lavor. The branch and prune algorithm for the molecular distance geometry problem with inexact distances. In Proceedings of the International Conference on Computational Biology (ICCB), volume 58, pages 349-353. World Academy of Science, Engineering and Technology, 2009.

[193] A. Mucherino, C. Lavor, L. Liberti, and E.-G. Talbi. A Parallel Version of the Branch \& Prune Algorithm for the Molecular Distance Geometry Problem. In Proceedings of the $8^{\text {th }}$ ACS/IEEE International Conference on Computer Systems and Applications (AICCSA). IEEE, Hammamet, 2010.

[194] A. Mucherino, L. Liberti, C. Lavor, and N. Maculan. Comparisons between an Exact and a MetaHeuristic Algorithm for the Molecular Distance Geometry Problem. In F. Rothlauf, editor, Proceedings of the Genetic and Evolutionary Computation Conference (GECCO), pages 333-340. ACM, Montreal, 2009.

[195] M. E. J. Newman. Fast algorithm for detecting community structure in networks. Physical Review E, 69(6):066133, 2004. 
[196] M. E. J. Newman. Finding community structure in networks using the eigenvectors of matrices. Physical Review E, 74(3):036104, 2006.

[197] M. E. J. Newman. Modularity and community structure in networks. Proceedings of the National Academy of Sciences of the U.S.A., 103(23):8577-8582, 2006.

[198] M. E. J. Newman. Networks: An Introduction. Oxford University Press, Oxford, 2010.

[199] M. E. J. Newman and M. Girvan. Finding and evaluating community structure in networks. Physical Review E, 69(2):026113, 2004.

[200] Y. Q. Niu, B. Q. Hu, W. Zhang, and M. Wang. Detecting the community structure in complex networks based on quantum mechanics. Physica A, 387(24):6215-6224, 2008.

[201] K. J. Nurmela and P. R. J. Östergård. Packing up to 50 Equal Circles in a Square. Discrete \& Computational Geometry, 18(1):111-120, 1997.

[202] P. Paatero. The Multilinear Engine: A Table-Driven, Least Squares Program for Solving Multilinear Problems, Including the $n$-Way Parallel Factor Analysis Model. Journal of Computational and Graphical Statistics, 8(4):854-888, 1999.

[203] M. Padberg and G. Rinaldi. Optimization of a 532-city symmetric traveling salesman problem by branch and cut. Operations Research Letters, 6(1):1-7, 1987.

[204] M. Padberg and G. Rinaldi. A Branch-and-Cut Algorithm for the Resolution of Large-Scale Symmetric Traveling Salesman Problems. SIAM Review, 33(1):60100, 1991.

[205] G. Palla, I. Derényi, I. Farkas, and T. Vicsek. Uncovering the overlapping community structure of complex networks in nature and society. Nature, 435(7043):814-818, 2005.

[206] R. G. Parker and R. L. Rardin. Discrete optimization. Computer Science and Scientific Computing. Academic Press, 1988.

[207] J. Puchinger and G. R. Raidl. Relaxation Guided Variable Neighbourhood Search. In Proceedings of the $18^{\text {th }}$ Mini Euro Conference on Variable Neighborhood Search (MEC-VNS), Tenerife, Spain, 2005.

[208] I. Quesada and I. E. Grossmann. An LP/NLP based branch and bound algorithm for convex MINLP optimization problems. Computers $\&$ Chemical Engineering, 16(10-11):937-947, 1992. 
[209] U. Raber. Nonconvex All-Quadratic Global Optimization Problems: Solution Methods, Application and Related Topics. PhD thesis, University of Trier, Germany, 1999.

[210] F. Radicchi, C. Castellano, F. Cecconi, V. Loreto, and D. Parisi. Defining and identifying communities in networks. Proceedings of the National Academy of Sciences of the U.S.A., 101(9):2658-2663, 2004.

[211] U. N. Raghavan, R. Albert, and S. Kumara. Near linear time algorithm to detect community structures in large-scale networks. Physical Review E, 76(3):036106, 2007.

[212] A. Ramani and I. L. Markov. Automatically exploiting symmetries in constraint programming. In B. Faltings, A. Petcu, F. Fages, and F. Rossi, editors, Proceedings of the Annual Workshop on Constraint Solving and Constraint Logic Programming (CSCLP), volume 3419 of Lecture Notes in Artificial Intelligence, pages 98-112. Springer, Berlin, 2005.

[213] J. Reichardt and S. Bornholdt. Statistical mechanics of community detection. Physical Review E, 74(1):016110, 2006.

[214] T. Richardson, P. J. Mucha, and M. A. Porter. Spectral tripartitioning of networks. Physical Review E, 80(3):036111, 2009.

[215] A. D. Rikun. A Convex Envelope Formula for Multilinear Functions. Journal of Global Optimization, 10(4):425-437, 1997.

[216] M. Rosvall and C. T. Bergstrom. An information-theoretic framework for resolving community structure in complex networks. Proceedings of the National Academy of Sciences of the U.S.A., 104(18):7327-7331, 2007.

[217] H. S. Ryoo and N. V. Sahinidis. Global optimization of nonconvex NLPs and MINLPs with applications in process design. Computers $\& 3$ Chemical Engineering, 19(5):551-566, 1995.

[218] S. Lehmann and L. K. Hansen. Deterministic modularity optimization. The European Physical Journal B, 60(1):83-88, 2007.

[219] H. Sachs. Coin graphs, Polyhedra, and conformal maping. Discrete Mathematics, 134(1-3):133-138, 1994.

[220] N. V. Sahinidis and M. Tawarmalani. BARON 7.2.5: Global Optimization of Mixed-Integer Nonlinear Programs, User's Manual, 2005.

[221] M. Sales-Pardo, R. Guimerà, A. A. Moreira, and L. A. N. Amaral. Extracting the hierarchical organization of complex systems. Proceedings of the National Academy of Sciences of the U.S.A., 104(39):15224-15229, 2007. 
[222] P. Schuetz and A. Caflisch. Efficient modularity optimization by multistep greedy algorithm and vertex mover refinement. Physical Review E, 77(4):046112, 2008.

[223] P. Schuetz and A. Caflisch. Multistep greedy algorithm identifies community structure in real-world and computer-generated networks. Physical Review E, 78(2):026112, 2008.

[224] J. Scott and M. Hughes. The Anatomy of Scottish Capital: Scottish Companies and Scottish Capital, 1900-1979. Croom Helm, London, 1980.

[225] K. H. Shafique. Partitioning a Graph in Alliances and its Application to Data Clustering. PhD thesis, University of Central Florida Orlando, Florida, 2004.

[226] N. Shah. Mathematical programming techniques for crude oil scheduling. Computers $\&$ Chemical Engineering, 20, Supplement 2:S1227-S1232, 1996.

[227] D. M. Shepard, M. C. Ferris, G. H. Olivera, and T. R. Mackie. Optimizing the Delivery of Radiation Therapy to Cancer Patients. SIAM Review, 41(4):721744, 1999.

[228] H. D. Sherali. Personal communication with L. Liberti, June 2007.

[229] H. D. Sherali, K. Ozbay, and S. Subramanian. The time-dependent shortest pair of disjoint paths problem: Complexity, models and algorithms. Networks, 31(4):259-272, 1998.

[230] J. Shi and J. Malik. Normalized Cuts and Image Segmentation. IEEE Transactions on Pattern Analysis and Machine Intelligence, 22(8):888-905, 2000.

[231] H. A. Simon. The Architecture of Complexity. Proceedings of the American Philosophical Society, 106(6):467-482, 1962.

[232] E. M. B. Smith and C. C. Pantelides. A symbolic reformulation/spatial branchand-bound algorithm for the global optimisation of nonconvex MINLPs. Computers $\& 3$ Chemical Engineering, 23(4-5):457-478, 1999.

[233] K. Stephenson. Introduction To Circle Packing: The Theory of Discrete Analytic Functions. Cambridge University Press, Cambridge, 2005.

[234] Y. Sun, B. Danila, K. Josić, and K. E. Bassler. Improved community structure detection using a modified fine-tuning strategy. Europhysics Letters, 86(2):28004, 2009.

[235] P. G. Szabó. Optimal Substructures in Optimal and Approximate Circle Packings. Beitrage zur Algebra und Geometrie (Contributions to Algebra and Geometry), 46(1):103-118, 2005. 
[236] P. G. Szabó, M. C. Markót, and T. Csendes. Global optimization in geometry - Circle packing into the square. In C. Audet, P. Hansen, and G. Savard, editors, Essays and Surveys in Global Optimization, pages 233-265. Springer, Berlin, 2005.

[237] P. G. Szabó, M. C. Markót, T. Csendes, E. Specht, L. G. Casado, and I. García. New Approaches to Circle Packing in a Square: With Program Codes (Springer Optimization and Its Applications). Springer-Verlag New York, Inc., Secaucus, NJ, USA, 2007.

[238] F. Tardella. Existence and sum decomposition of vertex polyhedral convex envelopes. Optimization Letters, 2(3):363-375, 2008.

[239] V. A. Traag, P. Van Dooren, and Y. Nesterov. Narrow scope for resolutionlimit-free community detection. Physical Review E, 84(1):016114, 2011.

[240] I. Tseveendorj. Reverse convex problems: an approach based on optimality conditions. Journal of Applied Mathematics and Decision Sciences, 2006(ID29023):1-16, 2006.

[241] H. Tuy. D.c. optimization: Theory, methods and algorithms. In R. Horst and P. M. Pardalos, editors, Handbook of Global Optimization, volume 1, pages 149-216. Kluwer Academic Publishers, Dordrecht, 1995.

[242] H. Tuy. Convex Analysis and Global Optimization. Kluwer Academic Publishers, Dordrecht, 1998.

[243] S. A. Vavasis. Nonlinear Optimization: Complexity Issues. Oxford University Press, Oxford, 1991.

[244] A. Wächter and L. T. Biegler. On the implementation of an interior-point filter line-search algorithm for large-scale nonlinear programming. Mathematical Programming, 106(1):25-57, 2006.

[245] S. Wasserman and K. Faust. Social Network Analysis: Methods and Applications. Structural Analysis in the Social Sciences. Cambridge University Press, 1994.

[246] D. J. Watts and S. H. Strogatz. Collective dynamics of 'small-world' networks. Nature, 393(6684):440-442, 1998.

[247] H. Wenqi and K. Yan. A heuristic quasi-physical strategy for solving disks packing problem. Simulation Modelling Practice and Theory, 10(3-4):195-207, 2002 . 
[248] T. Westerlund and F. Pettersson. An extended cutting plane method for solving convex MINLP problems. Computers $\&$ Chemical Engineering, 19, Supplement 1:131-136, 1995.

[249] D. Wu and Z. Wu. An updated geometric build-up algorithm for solving the molecular distance geometry problem with sparse distance data. Journal of Global Optimization, 37(4):661-673, 2007.

[250] G. Xu, S. Tsoka, and L. G. Papageorgiou. Finding community structures in complex networks using mixed integer optimisation. The European Physical Journal B, 60(2):231-239, 2007.

[251] W. W. Zachary. An Information Flow Model for Conflict and Fission in Small Groups. Journal of Anthropological Research, 33(4):452-473, 1977.

[252] W. Zhan, Z. Zhang, J. Guan, and S. Zhou. Evolutionary method for finding communities in bipartite networks. Physical Review E, 83(6):066120, 2011.

[253] W. Zhu. Unsolvability of some optimization problems. Applied Mathematics and Computation, 174(2):921-926, 2006. 